\title{
Optical surveys for space debris
}

\author{
Thomas Schildknecht
}

Received: 12 May 2006 / Revised: 29 August 2006 /

Published online: 9 January 2007

(C) Springer-Verlag 2007

\begin{abstract}
Space debris-man-made non-functional objects of all sizes in near-Earth space - has been recognized as an increasing threat for current and future space operations. The debris population in near-Earth space has therefore been extensively studied during the last decade. Information on objects at altitudes higher than about 2,000 km is, however, still comparatively sparse. Debris in this region is best detected by surveys utilizing optical telescopes. Moreover, the instruments and the applied observation techniques, as well as the processing methods, have many similarities with those used in optical surveys for 'astronomical' objects like near-Earth objects (NEOs).

The present article gives a general introduction to the problem of space debris, presents the used observation and processing techniques emphasizing the similarities and differences compared to optical surveys for NEOs, and reviews the results from optical surveys for space debris in high-altitude Earth orbits. Predictions on the influence of space debris on the future of space research and space astronomy in particular are reported as well.
\end{abstract}

Keywords Astrometry - Celestial mechanics · Minor planets, Asteroids · Methods: Observational · Techniques: Miscellaneous · Surveys

\section{Contents}

1 Introduction

2 Space debris

T. Schildknecht $(\bowtie)$

Astronomical Institute of the University of Bern, 3012 Bern, Switzerland

e-mail: thomas.schildknecht@aiub.unibe.ch

URL: www.aiub.unibe.ch 
2.1 What is space debris?

2.1.1 How is space debris generated?

2.1.2 How much space debris are there?

2.1.3 Temporal evolution

2.2 Why is space debris a problem?

2.2.1 May debris fall from sky?

2.2.2 Risks for space operations

2.3 What do we know today?

2.3.1 The USSTRATCOM catalog

2.3.2 Samples from radar observations

2.3.3 Optical observations

2.3.4 In situ measurements

2.3.5 Models

2.4 Protecting the space environment

2.4.1 Space debris mitigation .

2.4.2 International cooperation

2.5 Summary

3 Observation techniques

3.1 Characteristics of objects in high-altitude earth orbits

3.1.1 Basic characteristics of GEO orbits

3.1.2 Basic characteristics of GTO orbits

3.1.3 Apparent mean density of cataloged objects at the sky

3.2 Sky surveys for moving objects

3.3 Surveys for high-altitude space debris

3.3.1 Scanning of declination stripes

3.3.2 Masking technique

3.3.3 Exposure time

3.3.4 Overlapping

3.3.5 Multi-stripe scanning

3.3.6 Surveyed area and data rate per hour

3.3.7 Observational constraints

3.3.8 Astrometric calibration .

3.3.9 Orbit determination and correlation with catalog

4 The ESA survey for small-sized debris in high-altitude orbits .

4.1 Introduction

4.2 Observation campaign characteristics

4.3 Results

4.3.1 Absolute magnitude distribution

4.3.2 Inclination distribution

4.3.3 Distribution of semimajor axes

4.3.4 Inclination and right ascension of the ascending node

4.4 Surveys for objects in highly eccentric orbits

4.5 The high area-to-mass ratio population

5 Summary

\section{Introduction}

Optical surveys for space debris, the search for man-made debris in near-Earth space is a topic closely related to the search for near-Earth objects (NEOs) by optical methods.

Space debris are by definition non-functional, man-made objects in space, including large objects of several meters in size like defunct satellites and spent upper stages, but also including all sort of centimeter and millimeter-sized debris created by explosions and collisions, and even very small particles like 
paint flakes, and solid rocket motor slag or dust. These objects are populating the same regions in near-Earth space, which are used for operating satellites. Space debris are thus mostly found in geocentric orbits with altitudes ranging from 300 to $40,000 \mathrm{~km}$.

In optical surveys, space debris appear as fast moving objects with angular velocities ranging from a few arc seconds per second to more than 1,000 arc seconds per second with respect to the stellar background. Some space debris objects may appear as bright as magnitude zero but many are fainter than 20th magnitude depending on their illumination, distance, size and light reflection properties at the observation epoch. The resulting requirements for the optical sensors to be used in these surveys are strikingly similar to the corresponding demands for systems used in surveys for minor planets, comets and near-Earth objects. In particular, the survey telescopes should have fast optics with very large fields of view. As a consequence, the focal plane arrays become large. The high angular velocities of the objects of interest limit the exposure times to a few seconds and require frame rates of the order of several frames per minute. Mainly the latter makes the space debris surveys technically more demanding than surveys for NEOs. Very fast detector readout rates are mandatory and average data rates of many gigabytes must be handled and often processed in near real time.

In the near future, there are a series of large synoptic survey systems coming up, like the Panoramic Survey Telescope and Rapid Response System (PanSTARRS), the Large Synoptic Survey Telescope (LSST), the Visible and Infrared Survey Telescope for Astronomy (VISTA), etc. In order to succeed, these projects will have to combine and enhance the most advanced hardware and techniques developed in both, the field of NEO surveys and the field of optical space debris surveys including space surveillance. This will include specialized hardware like advanced detectors developed for surveillance applications, advanced image processing algorithms, techniques to link up observations from moving objects and to determine their orbits, and finally sophisticated data archiving and cataloguing techniques.

The processing pipelines of space debris surveys contain algorithms to detect faint, moving objects, to determine their orbits and estimate their physical size, and eventually to catalog these objects. All of these processing steps are found in pipelines used for minor planet and NEO surveys, as well. In both cases, the large number of newly discovered objects requires new techniques to efficiently cross-correlate observations from different nights and/or sites, to uniquely identify identical objects and, finally, to correlate these with a catalog of known objects.

A lot of effort has been devoted to set-up and run large NEO surveys during the last decade. Some surveys like the LONEOS, the Catalina (Larson et al. 1998), and part of the NEAT (Pravdo et al. 1999), are using classical wide field Schmidt telescopes, which were refurbished and equipped with CCD detectors (charge-coupled devices). The Spacewatch program, which pioneered the automated surveys using CCDs, is concentrating on deep surveys with a large telescope but a moderate field of view with particular interest in detecting 
transneptunian objects (TNOs) (Larsen et al. 2001). The most productive survey, the LINEAR, is, however, using technology which was developed by the US Air Force for space surveillance, i.e., to detect and track man-made objects in near-Earth space (Stokes et al. 2000)! Vice versa, the NEAT system in Maui has been successfully used to survey high-altitude artificial objects in space (Africano et al. 2001). One system, the Bisei Space Guard Center (BSGC), consisting of 0.5 and $1-\mathrm{m}$ telescope has been conceived as combined NEO and space debris survey system already from the beginning (Isobe et al. 2004, 2000). A comprehensive review on the NEO programs may be found in Stokes and Evans (2002).

This review starts with a general introduction into the problem of space debris (Sect. 2). Brief overviews on the risks posed by space debris, on the measurements performed to assess the current space debris population, on the models and finally on the mitigation measures are given. In this section we also briefly review and summarize the results from optical surveys of space debris. We restrict this review to published results, but note that there are many groups, which sometime intended to perform some optical observations of space debris.

Section 3 is devoted to the survey techniques for high-altitude space debris. We will limit the survey region to the so-called geostationary ring (also called geostationary Earth orbit, GEO) and to the region of so-called geostationary transfer orbits (GTOs). The characteristics of these two orbital regimes, in particular from the observational point of view, will be presented. This section is focusing on the techniques used in the ESA space debris surveys, but techniques used in other space debris surveys will be mentioned and similarities with NEO surveys emphasized.

The essential results from the ESA space debris survey-the largest and deepest survey for space debris at high altitudes-are presented in Sect. 4. A comparison with results from other surveys is done as far as possible.

\section{Space debris}

The space age, which has been euphorically initiated on 4 October 1957 with the start of the first Russian spacecraft Sputnik1, has become reality today. In our daily life we constantly - indirectly or directly - make use of products from space research. Cost effective and reliable intercontinental phone and data connections and countless numbers of TV channels are only possible thanks to communication satellites orbiting at an altitude of $36,000 \mathrm{~km}$. In the meantime - and perhaps outside of the public's perception - the achievements of the space age have a great impact on many sectors of our daily life and in some cases became even indispensable.

For the general public, space research is primarily associated with manned and scientific space missions. Both, although being essential parts of space research, currently are of comparably minor importance for immediate practical applications. But large parts of transport on land, sea and air rely on satellitebased navigation systems. Weather forecasts, just to name another daily live 
example, are substantially based on data from weather satellites as well as Earth observation satellites.

Space research undoubtedly generated a wide variety of useful applications and thereby shaped our modern societies and will continue to play a role in future. From experience we know that many novel applications, which are not at all foreseeable today, may become reality within a few years only. It is therefore imperative that we make use of space in a way that future generations still may use this invaluable resource and take profit from it.

However, do we really exploit the resource 'outer space' in a sustainable way? In other words, are space operations still possible in the foreseeable future if we continue to exploit space in the same way as we do today? Unfortunately, there are indications that this will not be the case. The 'space age' could perhaps already be terminated in a few decades! Impacts from artificial particles on the US space shuttle, on solar panels of satellites (Sect. 2.2.2), etc. may lead us to the question: 'Did we already fill up the near-Earth space with artificial debris in a way that space becomes too crowded for future manned and unmanned missions?'

Space operations since the start of the first artificial Earth satellite have inevitably produced some debris, also called 'space debris' or 'orbital debris', and left them in near-Earth space. During each launch only a very small fraction of the total mass brought into space actually consists of the active payload. By far the largest part of the mass actually becomes space debris within a few minutes. Today's dispensable launchers in most cases leave upper stages, payload shrouds, adapter rings, etc. in an Earth orbit. Also, the actual payload eventually will end up as space debris after it has accomplished its mission. It is therefore not surprising that the currently, roughly 600, active satellites comprise only about $5 \%$ of the total number of known artificial objects in space larger than about $10-20 \mathrm{~cm}$. The mentioned impacts on the space shuttle and on other objects retrieved from space stem (fortunately!) from much smaller particles with diameters ranging from a few hundredths of a millimeter to a few millimeters. Such objects are generated mostly by explosions of satellites and spent upper stages. Weathered paint on exposed surfaces and dust from solid rocket motors are other sources of very small particles. There are also natural micrometeorites in the near-Earth space environment. In this environment, however, space debris are by now already outnumbering micrometeorites by far for all sizes except a small range between 10 and $100 \mu \mathrm{m}$ where the populations may be equal in number (see Fig. 18).

In an attempt to reduce the collision risk for the international space station ISS and the US space shuttle their trajectories are constantly correlated with the orbits of known objects. As a consequence several times a launch had to be postponed or collision avoidance maneuvers in orbit had to be performed. But also unmanned spacecrafts like, e.g., the European ERS Earth observation satellites actively changed their orbits to avoid collision with space debris. Moreover, the ISS has been equipped with passive shields weighing several tons in order to protect the station from impacts of particles with sizes up to a few millimeters. 
What do we know about the current space debris environment? How many pieces of which sizes and in which orbits are there? Questions, which today we are able to answer in an incomplete way only. In the low Earth orbit (LEO) region, which is defined as the region up to $2,000 \mathrm{~km}$ altitude, we believe to know the orbits of most artificial objects larger than about $20 \mathrm{~cm}$. For smaller pieces there is only limited statistical information, like the number and size of pieces in particular orbit regions. At higher altitudes the information becomes sparser. In the so-called geostationary ring (GEO), i.e., the altitude where satellites apparently remain stationary with respect to an Earth fixed frame and where most of the communication satellites reside, a substantial population of debris objects in the size range of $0.1-1 \mathrm{~m}$ has been discovered only recently. There is no knowledge at all about smaller fragments. In the region of the so-called geostationary transfer orbits (GTOs), orbits with a closest approach to the Earth (perigee) at altitudes below $2,000 \mathrm{~km}$ and a farthest point (apogee) at the geostationary altitude of $36,000 \mathrm{~km}$, our knowledge is even limited to objects larger than about $1 \mathrm{~m}$. Figure 1 shows a snapshot view of the about 9,000 'large' objects with known orbits as seen from a vantage point in a distance of 15 Earth radii above the North pole. In a dense shell around the Earth we recognize the objects in low orbits (LEO). Objects in GEO form a distinct ring whereas the objects lying in between are mostly either on GTO or on highly inclined elliptical orbits. Many of the latter are Russian communication satellites in the class of the so-called Molniya orbits which are highly elliptical with an inclination of $63^{\circ}$ and with their apogee above the northern hemisphere. Objects in such orbits stay, for most of the time, over high northern latitudes and are used for communication in these regions.

In recent years, the scientific investigation of the space debris problem has become an independent research topic within the field of space research. Observation programs to investigate the current space debris population have been initiated, and at the same time models to understand the sources and the evolution of the population were developed. It is evident that the creation of space debris, in particular by explosions, must be drastically reduced if we want to preserve the near-Earth space for future space activities a few decades from now. Space debris, once created, cannot be collected-at least there are no feasible technical solutions available in foreseeable future.

\subsection{What is space debris?}

The International Academy of Astronautics (IAA) has defined the term orbital debris (being a synonym to space debris) in its position papers of 1993 (IAA position paper on orbital debris 1993) and 2001 (IAA position paper on orbital debris 2001) in the following way.

'Orbital debris is herein defined as any man-made object which is non-functional with no reasonable expectation of assuming or resuming its intended function, or any other function for which it is or can be expected to be authorized, including fragments and parts thereof.' 


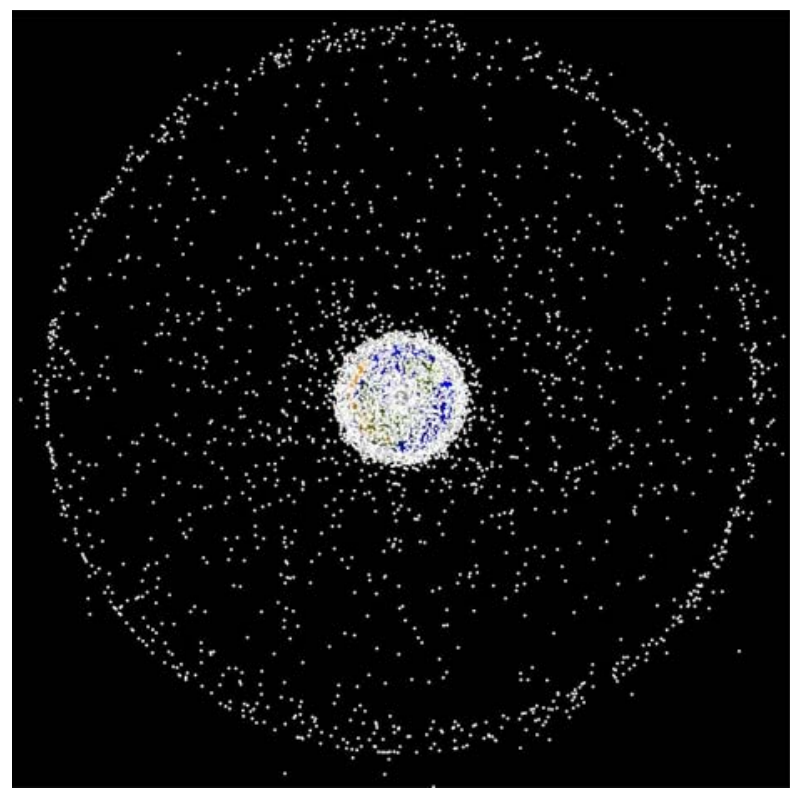

Fig. 1 Snapshot view of the about 9,000 'large' objects with known orbits as seen from a vantage point in a distance of 15 Earth radii above the North pole

From the legal appeal of this text we may already guess that the achievement of an international agreement on the mitigation of space debris is not at all a trivial task. At this point strong economical interests as well as questions concerning the legal liability of states become the driving moments.

\subsubsection{How is space debris generated?}

The ultimate source of all kind of space debris is, of course, the insertion of material in an orbit, i.e., the launch of any material from Earth. Figure 2 shows the annual numbers of successful launches into orbit since the start of the space age in 1957. Although the number of launches has significantly decreased during the recent years this does not imply that the total mass put into orbit is also decreasing. Launchers became more powerful and in many cases insert more than one satellite into orbit. Each launch is generally disposing several tons of material in orbit. A large part of the mass is concentrated in the form of spent upper stages and payloads. However, in terms of number of objects the smaller pieces predominate. Even before a satellite reaches its final orbit and may start its intended operation a variety of so-called 'mission related objects' may be freed: payload shrouds, adapter rings which fix the payload on the launcher, explosive bolts, instrument covers, etc., to name only a few of them.

If solid rocket motors are used as upper stages, the solid exhaust products of these motors contribute to the space debris population, as well. Although most of the burning products (aluminum oxide) are released in the form of 


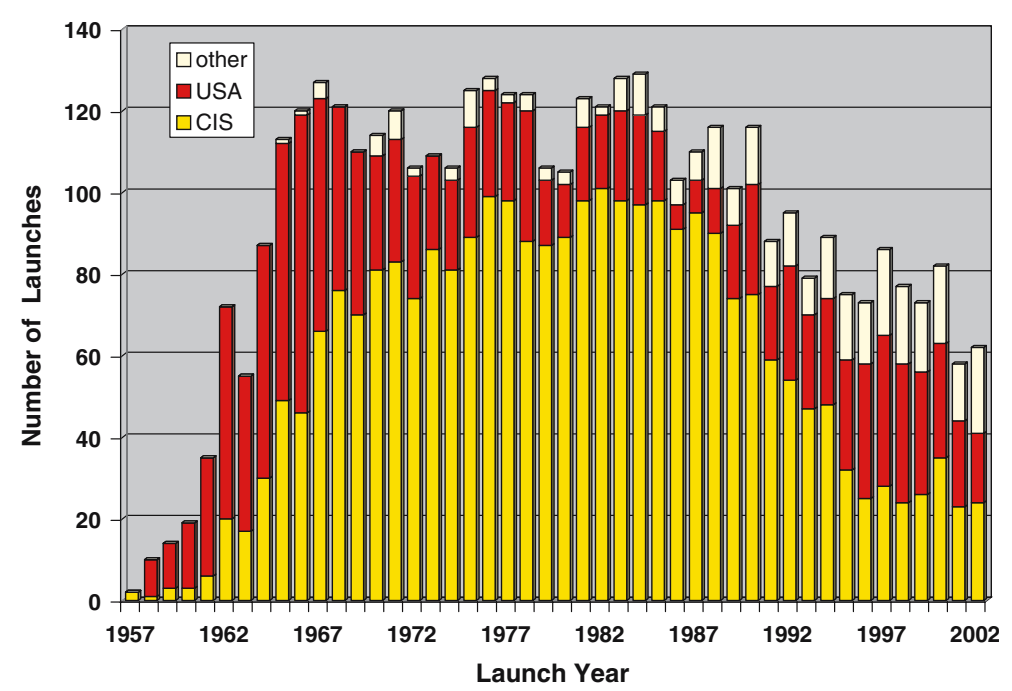

Fig. 2 Annual numbers of successful launches into orbit since the start of the space age in 1957 (ESA 2003)

dust with particle diameters of a few micrometers, the so-called slag particles with diameters ranging from millimeters to centimeters are produced especially towards the end of the burning phase.

The surfaces of objects in space are exposed to a harsh environment. Paint layers, for example, are gradually deteriorated by hard UV radiation and oxidization by residual air molecules and atoms plays an important role in low orbits. As a consequence of these aging processes the layers may 'peel off' and generate myriads of small paint flakes with sizes in the millimeter and sub millimeter range. Other materials like thermal insulation blankets made from composite materials may also be disintegrated and released in the form of small particles.

By far most of the particles with diameters larger than a few centimeters, however, are generated by in-orbit explosions. Until today more than 170 in-orbit explosions have been recorded, involving rocket upper stages, auxiliary motors, but also satellites. Explosions may be triggered by several mechanisms: (1) by the failures of an internal component containing stored energy, e.g., batteries, (2) by the ignition of residual fuel, (3) by the burst of a pressurized vessel or (4) by the intentional ignition of a self destruction mechanism. In principle, each source of energy in an object may sooner or later lead to an explosion. Rocket upper stages, for example, usually contain some residual fuel at the end of their mission (some reserve is preferred rather than becoming short of fuel). This residual fuel may cause harm even after years in space: in February 1992 a rocket upper stage launched in 1968 exploded after having spent more than 23 years in space! Among the common upper stages types there exist (or existed) some notorious candidates for such events. Some of these types, among them also the European Ariane upper stages, are nowadays so-called 
passivated, which means that their residual fuel after the end of the mission is either vented into space by opening a valve or burned through a so-called depletion burn. This procedure seems to be successful, at least until today there were no explosions of passivated upper stages observed. Satellites are candidates for unintended explosions, as well. A short circuit, for example, or an uncontrolled charging of batteries after the 'switch off' of a satellite may lead to explosions. Some intentional explosions took place during the cold war era in the framework of anti-satellite weapon tests, or in order to hide the technical capabilities by self-destructing sensitive satellites. Fortunately, such experiments were no longer continued in recent years.

A few well-known single events have significantly enhanced the space debris population. A prominent example is the explosion of a small airborne 'Pegasus' launcher in June 1996, which was brought into space 2 years earlier. The object made from carbon reinforced composite material thereby disrupted in countless small, wire-shaped, fragments. The 700 observed objects suddenly enhanced the space debris population in $600 \mathrm{~km}$ altitude by at least a factor of two. Another 'detective story' started about 14 years ago when a population of centimeter-sized objects was found at $850-1000 \mathrm{~km}$ altitude (Kessler et al. 1997). The number of these pieces is estimated to be about 80,000 ! Detailed radar measurements supplemented by optical observations soon indicated that these are swarms of small metallic spheres or droplets. The orbits of the pieces moreover pointed to a class of Russian reconnaissance satellites as the possible sources of the swarms. In the mid 1990s the objects were identified unambiguously as droplets of a metallic cooling liquid (NaK) from Russian 'RORSAT' radar ocean reconnaissance satellites. The RORSATs operated at altitudes of $250-270 \mathrm{~km}$ and contained a nuclear reactor to provide power to the energy intensive radar. In order to prevent the reentry of the radioactive material (or at least to postpone it for a significant amount of time) which would contaminate the environment on ground, as did COSMOS 954 in 1978, the satellites were re-boosted into orbits at $900-950 \mathrm{~km}$ altitude at the end of their lifetime. This operation was followed by an ejection of the reactor fuel rods in order to 'passivate' the system (Fig. 3). This latter operation most likely dispersed the coolant of the primary cooling circuit (up to 13 liters per event).

The space debris population may, of course, also grow through mutual collision among debris pieces or through collision of debris with natural objects (micrometeorites). This mechanism might play some role for very small particles, e.g., if ejecta are produced when a micrometeorite or a debris particle hits a satellite. Today, larger debris pieces fortunately collide with each other with a vanishing small probability only. This could, however, change dramatically if the density of objects continues to increase!

\subsubsection{How much space debris are there?}

We already mentioned that we currently know the orbits of about 9,000 'larger' man-made objects in space. In the LEO environment this catalog of orbits probably contains most of the man-made objects larger than about $20 \mathrm{~cm}$. 


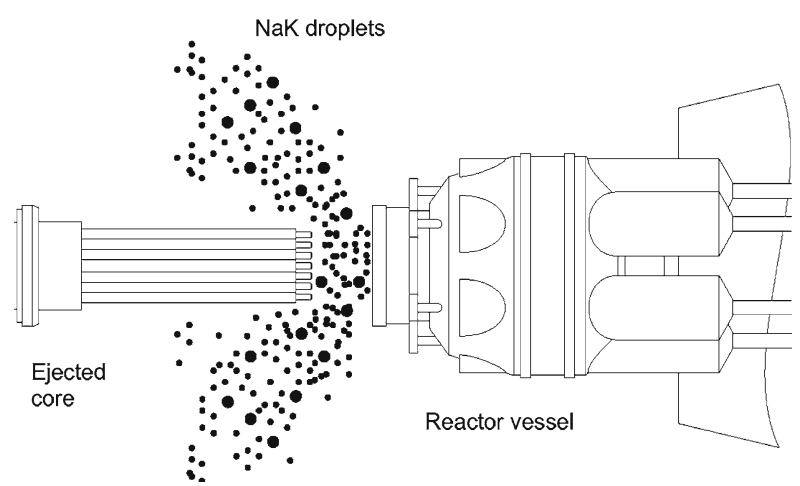

Fig. 3 Liquid coolant dispersed into space when RORSAT reactor cores were ejected (from Bendisch et al. 2002)

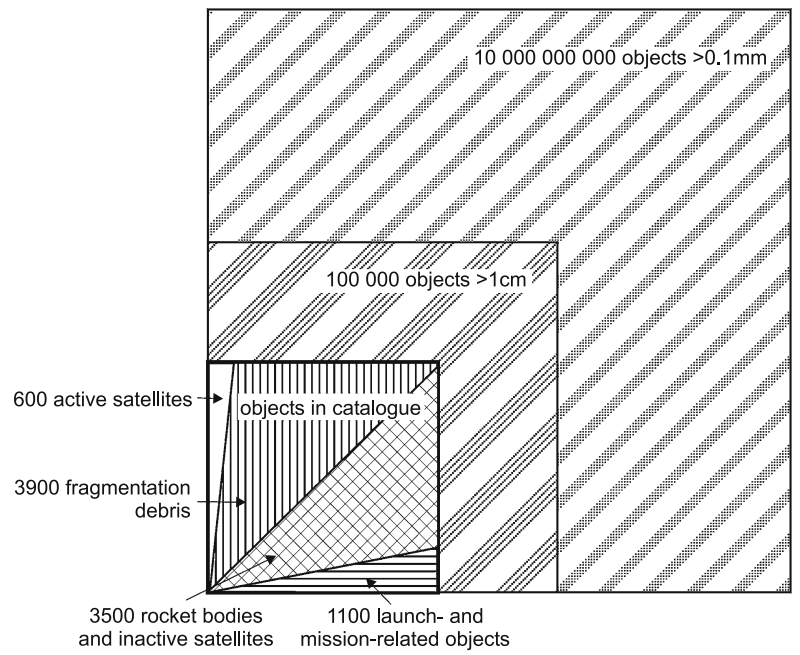

Fig. 4 Estimated space debris population according to object sizes and types (status 2000; note logarithmic scale)

The corresponding limit for orbits at higher altitudes is about $1 \mathrm{~m}$. Only about $5-6 \%$ of these objects are operational satellites (see Fig. 4). The mission related objects contribute about $12 \%$ and fragments from explosions over $40 \%$ to the total population of known objects. For objects with sizes from 1 to $20 \mathrm{~cm}$ in LEO, we are forced to use extrapolation methods based on statistical snapshot samples. Based on radar measurements this population is estimated to consist of at least 100,000 debris pieces. Probably there exist more than ten billion debris larger than $0.1 \mathrm{~mm}$, where these estimates have a large uncertainty.

\subsubsection{Temporal evolution}

When observing the temporal evolution of a debris cloud, e.g., shortly after an explosion, we discover that the fragments are dispersed quickly over a large 

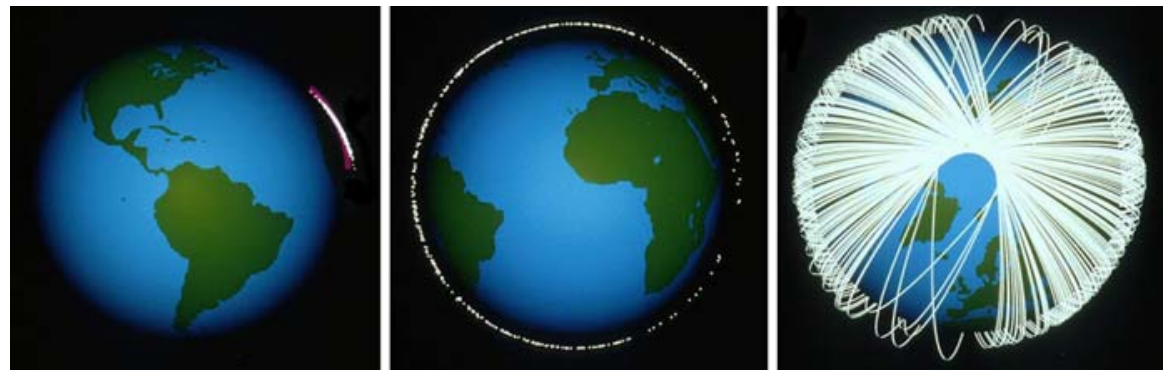

Fig. 5 Simulated explosion in LEO; immediately after the explosion (left), after some hours (middle), and after 3 years (right) (from ESA)

volume in space. Figure 5 shows a simulation of an explosion in a LEO. The simulation ejected the fragments according to the expected velocity and mass distributions uniformly into all directions. The laws of celestial mechanics tell, however, that the cloud is not immediately expanding into a spherical shell, but that the fragments in a first stage are distributed along the orbit of the parent body. Figure 5 shows the cigar-shaped distribution of the fragments immediately after the explosion on the left side. After several hours the debris cloud has expanded into a toric region along the parent orbit (Fig. 5 middle). The figure on the right shows the situation 3 years after the event. The fragments are now dispersed in an almost closed shell around the globe. The dispersion is caused by the perturbing forces of the gravity field (mainly due to the equatorial bulge), which force the orbital planes to precess, but with a slightly different rate for the original slightly different orbits of the fragments. This mechanism is responsible for the fact that each debris cloud in LEO is sooner or later causing a global 'pollution' roughly at the height of the original orbit.

Do the fragments stay 'forever' in this shell? Fortunately, there is, at least in low altitude orbits, a natural 'sink' for space debris: the atmosphere. All objects in orbits below about $1,000 \mathrm{~km}$ of altitude constantly collide with residual air molecules and atoms. As a consequence of this so-called air drag the object is decelerated and loses kinetic energy, which eventually leads to the decay of the orbit. During the reentry into the atmosphere the drag from the denser air layers heats the object by friction and it eventually, with the exception of very massive objects (see below), burns up. The deceleration rate depends on the density of the air and thus strongly on the altitude. An additional important factor is the area-to-mass ratio of the object: 'lightweight' pieces with a comparable large area are subject to a stronger deceleration than 'massive', compact objects. At altitudes up to $600 \mathrm{~km}$ objects without an active propulsion system will reenter the atmosphere within a few months up to several years. For objects starting at altitudes of $600-800 \mathrm{~km}$ it takes several decades before they burn up in the atmosphere. Objects in orbits at altitudes above $800 \mathrm{~km}$ remain in orbit for several hundred years. Above altitudes of a few $1,000 \mathrm{~km}$, in particular in GEO, orbits have an 'indefinite' lifetime and objects will stay there 'forever'. 


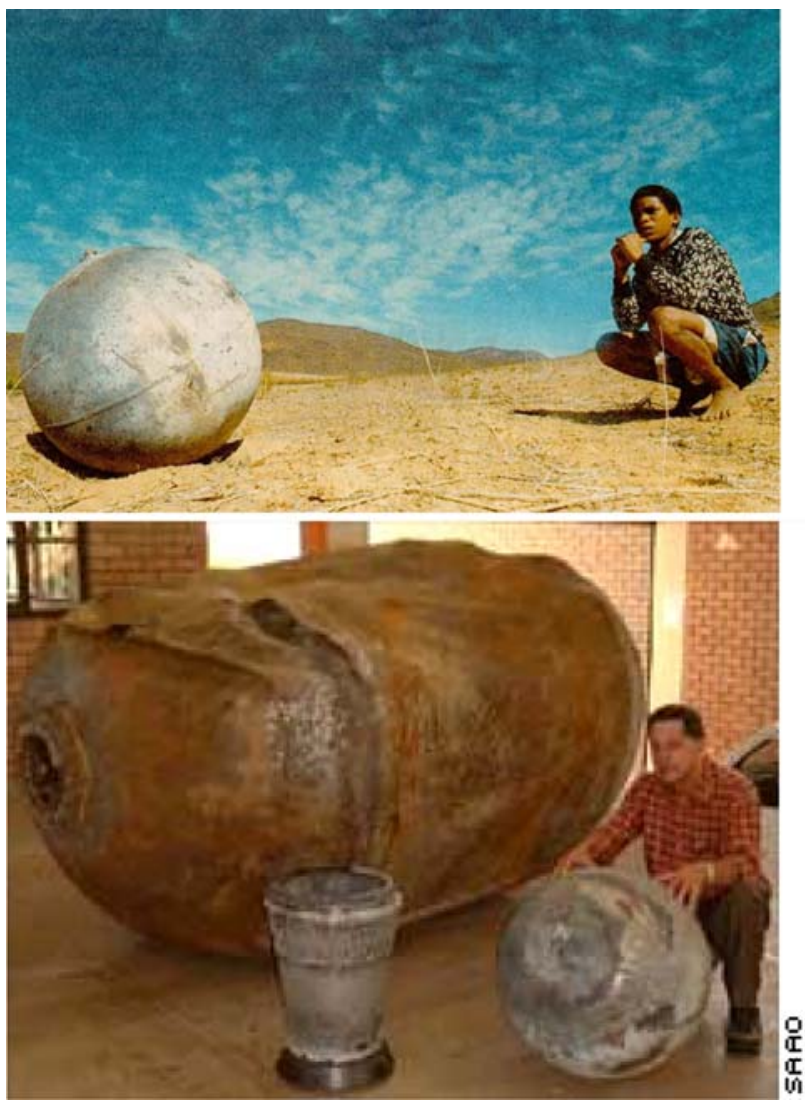

Fig. 6 Parts of a Delta II upper stage, which survived the atmospheric reentry

\subsection{Why is space debris a problem?}

\subsubsection{May debris fall from sky?}

Currently about one known 'larger' man-made object (out of the mentioned catalog of 9,000 known objects) 'falls' back to Earth every day. In addition, of course, many unknown smaller debris pieces and micrometeorites reenter the atmosphere. Satellites and rocket upper stages with a total mass of less than five tons in most cases burn up totally during their passage through the atmosphere. In most cases, there are exceptions. In January 1997 a piece of a Delta II upper stage - a fuel tank of $250 \mathrm{~kg}$ made from stainless steel - survived reentry and crashed down in Texas (USA). A similar tank, a spherical pressurized vessel and the nozzle of a rocket motor fell from the sky over South Africa in January 2000 (Fig. 6). As impressive as these examples are, the risk to be hit by a falling debris is vanishingly small and amounts to about one part per trillion per human per lifetime (UN technical report on space debris 1999). The risks of our daily life are comparably huge: the risk to be killed by a car accident amounts to about 
Table 1 Potential damage caused by impacts of small particles for the example of the US space shuttle

\begin{tabular}{ll}
\hline Object diameter $(\mathrm{mm})$ & Damage \\
\hline 0.04 & Craters in windows \\
0.1 & Penetration of EVA suits \\
0.5 & Penetration of radiators on the inner side of the \\
& payload doors (used for shuttle thermal control) \\
1 & Damage of the reinforced wing noses \\
5 & Penetration of the pressurized cabin \\
\hline
\end{tabular}

$1 / 100$ in industrialized countries, the one for death by fire is about $1 / 1,000$, and with a probability of $1 / 1,500,000$ we are deadly hit by lightning.

The small risk for casualties due to falling space debris assumes that objects with masses larger than five tons - the currently accepted international limit for so-called 'risk objects'-are actively de-orbited and brought to ground in a controlled way over an ocean. The latter has been successfully performed several times, e.g., in the year 2000 with the 14 ton astronomy satellite 'gamma ray observatory', and 1 year later with the Russian space station MIR which both fell into the pacific ocean. The indicated factor does not include any risks which may arise from radioactive material entering the atmosphere - a delicate topic which we will not further consider in this work.

In conclusion, we may state that space debris, with the exception of a few risk objects, which require a controlled de-orbiting, does not create any substantial risk neither for humans nor for the biosphere in general. The release of a few metric tons of gas into the atmosphere from the objects burning up during reentry is totally negligible, except for radioactive material.

\subsubsection{Risks for space operations}

Space debris first of all are a risk for the space missions. The debris pieces are mainly a threat due to their high relative velocity with respect to other objects in orbit. In LEO objects move with velocities of $7-8 \mathrm{~km} / \mathrm{s}$ (about $27,000 \mathrm{~km} / \mathrm{h}$ ) with respect to an Earth fixed frame. Consequently, the released energy during a collision is remarkably high. The kinetic energy of a small aluminum sphere of $1 \mathrm{~mm}$ diameter traveling at $10 \mathrm{~km} / \mathrm{s}$ is about equal to the kinetic energy of a bowling ball traveling at $520 \mathrm{~km} / \mathrm{h}$ ! On the average, objects in LEO have relative velocities of about $10 \mathrm{~km} / \mathrm{s}$, and in case of a head-on collision the relative velocities may even reach $16 \mathrm{~km} / \mathrm{s}$.

Table 1 summarizes the potential damages caused by impacts of small particles using the US space shuttle as an example. This example illustrates how impacts of even small particles may cause large damage with potentially catastrophic consequences. The fact that such impacts really take place is confirmed by the detailed inspections of the shuttle after each mission. On the average one window has to be replaced after each flight due to impacts of small particles. Figure 7 shows the recorded impacts on the shuttle payload door after the 


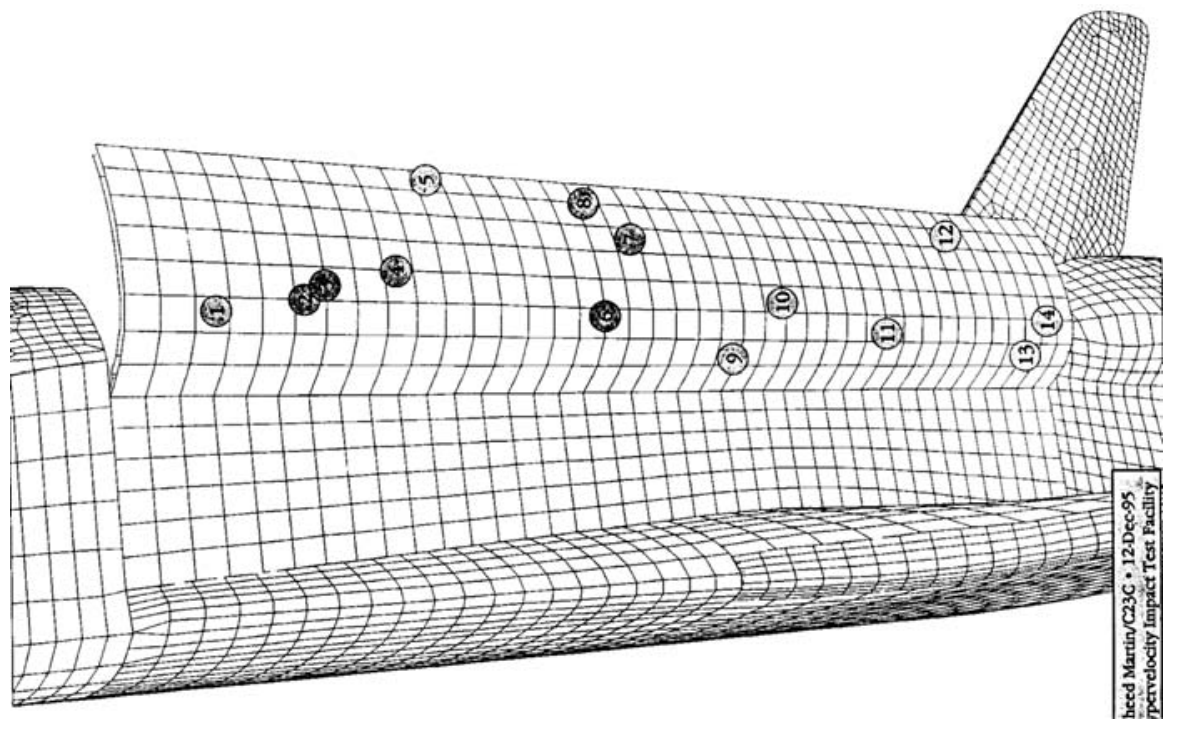

Fig. 7 Impacts on the US space shuttle payload door recorded after the STS-73 mission in 1995 (NASA)

mission STS-73 (16 days, 1995). In some of the 14 craters residuals of the corresponding projectile were found and their chemical composition was analyzed. A significant part thereof could be identified as space debris. This is why simulations are performed before each shuttle mission in order to assess the risk posed by the debris and the natural micrometeorite environment. Throughout the flights the shuttle is as often as possible oriented with its main engines pointing into the flight direction in order to best protect the crew and the critical systems. During extravehicular activities (EVAs) the entire shuttle is used as a shield to protect the EVA crew.

The threat for the international space station ISS is, due to its large cross section and long-term exposure to the environment, by orders of magnitudes larger than for the space shuttle. Over its entire lifetime we must expect impacts, which punctuate a pressurized module! The most endangered regions of the station were therefore identified using space debris models and equipped with dedicated shields. But even the most sophisticated shields are not able to protect the station from projectiles larger than a few millimeters. Consequently, scenarios and corresponding tools had to be provided to evacuate the station in an emergency situation and to perform some emergency repairs of holes in the walls of the pressurized compartments. Moreover, the station will be actively moved out of the trajectories of known objects. But there is still a risk from the particles in the size range from 1 to $20 \mathrm{~cm}$, which can be neither blocked by shields nor circumvented by an avoidance maneuver because their orbits are unknown.

On 24 July 1996 the first known collision between an active satellite and a known space debris took place. At this time the French satellite 'CERISE' was 

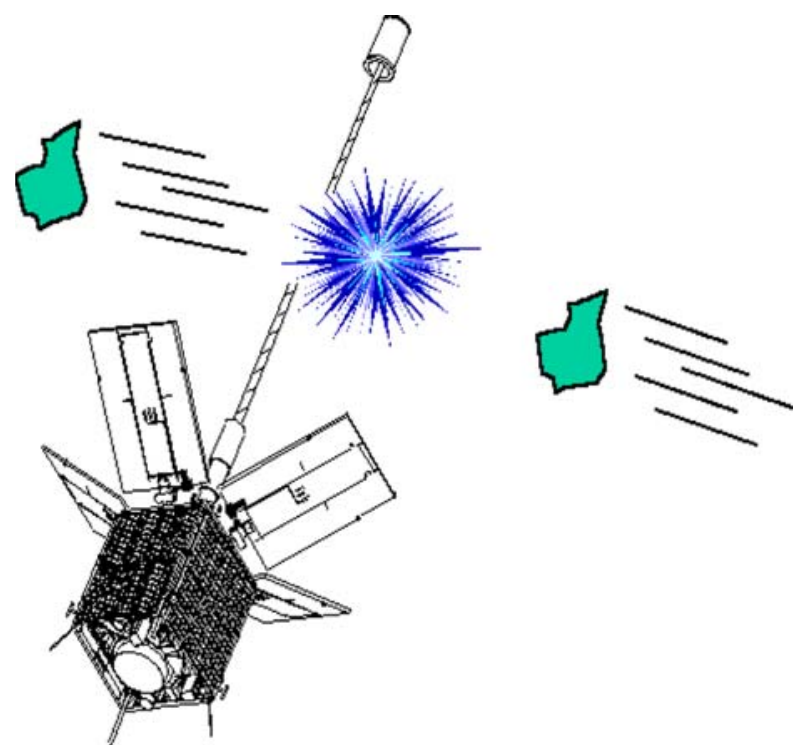

Fig. 8 The French satellite CERISE was hit by a known debris fragment from an Ariane upper stage on 24 July 1996. The fragment severed a boom (the satellite survived!)

hit by a fragment of an Ariane upper stage. The fragment was known (in the catalog of 9,000 objects), had a diameter of roughly $30 \mathrm{~cm}$ and originated from one of the mentioned explosions of a rocket upper stage. Fortunately not the satellite body was hit but only a long boom - a so-called gravity anchor used for attitude stabilization - was severed (Fig. 8). The satellite survived the collision, which took place with a relative velocity of over $14 \mathrm{~km} / \mathrm{s}$, in relatively good shape and continued its mission after reprogramming of the on-board computer to perform attitude control without the gravity anchor.

These examples illustrate that the small debris pieces in the size range from 0.1 to $100 \mathrm{~mm}$ may pose a substantial risk for manned and unmanned space missions. The critical property of the space debris population in a given region is therefore not the total mass of debris but rather the number of particles in a given size range. This in turn justifies the efforts to investigate the current population of small debris and to limit their generation in future primarily through the prevention of explosions.

\subsection{What do we know today?}

\subsubsection{The USSTRATCOM catalog}

The US Strategic Command (USSTRATCOM), a military entity of the USA, maintains a catalog of about 9,000 known 'large' objects. (Russia maintains a similar catalog but we restrict our description to the US catalog.) Sensors of the so-called 'space surveillance network' (SSN) continuously collect 

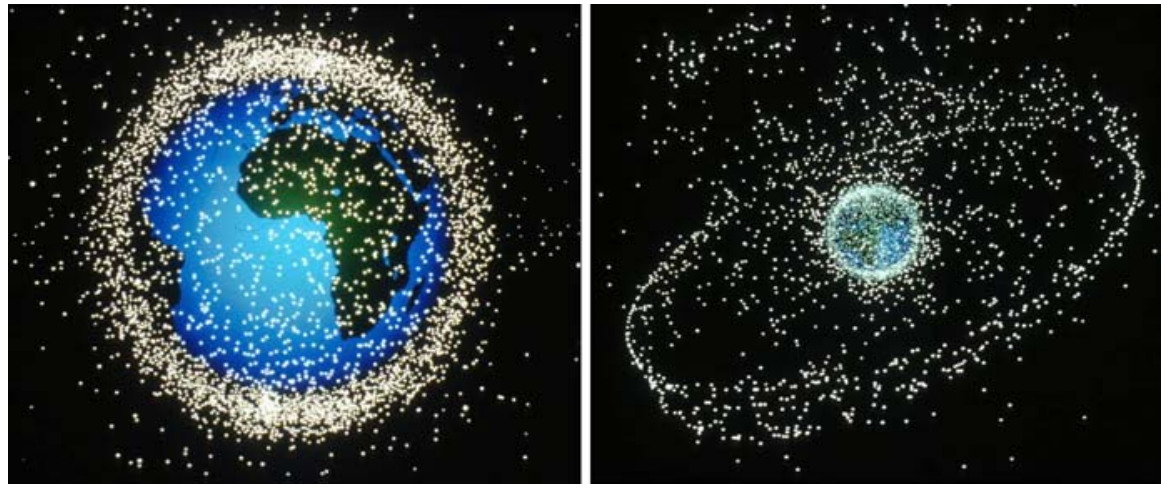

Fig. 9 Distribution of cataloged objects. LEO region on the left, high-altitude region as seen from a distance of 15 Earth radii on the right (ESA)

measurements, which are then combined with earlier data to produce orbits. The SSN consists of a global network of radar stations and a series of optical telescopes. Most radar systems are part of the US early warning system for intercontinental ballistic missiles and are capable of detecting new objects and of tracking known objects. Radar is mostly used to survey the LEO region. In LEO the SSN is thereby detecting and tracking all objects larger than about 10 $20 \mathrm{~cm}$. To observe objects at higher altitude, in particular including the GEO, comparatively cost effective optical systems outperform the radars. For high altitudes the SSN is using optical telescopes of $1 \mathrm{~m}$ aperture which are equipped with electro-optical sensors, the so-called Ground-based Electro-Optical Deep Space Surveillance system (GEODSS). The smallest objects, which these systems can detect in GEO, have sizes of the order of $1 \mathrm{~m}$ in diameter.

The continuous maintenance of the orbital element catalog (in real time!) with more than 10,000 objects is a non-trivial task, because objects which are not observed regularly become 'lost' after a relatively short time interval. It is not precisely known to what extent the catalog is complete, or in other words, which percentage of existing objects of a given size are really contained in the catalog. Moreover, the publicly available version of the catalog does not contain the so-called classified objects, as well as objects which could not be associated with a specific launch (e.g., debris pieces of unknown origin).

Figure 9 is complementing Fig. 1 and illustrates the distribution of the cataloged objects from two additional vantages points. The figure on the left-hand side illustrates the distribution of 'large' objects in LEO. The concentration over the poles is due to objects in high inclination orbits, which cross each other in the vicinity of the poles. An additional view from a distance of 15 Earth radii is given on the right-hand side. The geostationary ring is clearly visible, as well as a series of objects in inclined, highly eccentric orbits with their apogee over the northern hemisphere.

The USSTRATCOM catalog, although generated for a different purpose and known to be incomplete, is extensively used in space debris research. 


\subsubsection{Samples from radar observations}

Radar has proved to be highly efficient to detect and track small objects in LEO. There are basically two types of radars used for this purpose: (1) radars with a narrow field of view (i.e., a narrow beam) controlling their viewing direction mechanically using a parabolic dish antenna and (2) the so-called phased array radars with electronically controlled beam direction and a wide field of view. The latter types are ideal for survey purposes as they can detect and track multiple objects simultaneously in a field of view as large as $120^{\circ}$. The primary sensors in the SSN are of this second type. Due to their wide field of view, however, their sensitivity is considerably lower compared to the sensitivity of dish antennas with similar antenna sizes. Parabolic dish antennas, on the other hand, may usually track single objects only in a narrow field of view with a diameter of less than $1^{\circ}$. Such radars, however, are sensitive enough to cover the mentioned critical range of object sizes between a few millimeters and $20 \mathrm{~cm}$ in LEO. For space debris searches the systems are used in a so-called beam-park mode where the viewing direction is kept fixed and objects passing the field of view are recorded. These measurements give only statistical information by their nature. But nevertheless, these statistical samples contain information on the number and size of the detected objects as well as an approximate altitude and inclination of their orbits.

Since the beginning of the 1990s NASA has been intensively using the 36-m Haystack dish antenna in Massachusetts near Boston for their debris monitoring program (Settecerri et al. 1999) (Fig. 10). ESA initiated a similar program a few years later using the German 34-m dish radar facility at the Forschungsgesellschaft für Angewandte Naturwissenschaften (FGAN) near Bonn (Mehrholz et al. 2004). Both instruments have comparable performance and are able to detect objects with a diameter of $0.5-1 \mathrm{~cm}$ at an altitude of $1,000 \mathrm{~km}$.

\subsubsection{Optical observations}

Space debris can be detected with optical telescopes when the objects are illuminated by the Sun while the sky background is dark. For the LEO region this is only the case during $1-2 \mathrm{~h}$ after sunset and before sunrise. Most optical telescopes have a narrow field of view of the order of $1^{\circ}$ or less like parabolic dish radars. Their big advantage over radars, however, resides in their higher sensitivity in terms of object size at large distances: while the radar sensitivity falls off proportional to the distance to the fourth, the sensitivity of optical instruments is only reduced proportional to the distance squared. This is the main reason why optical telescopes are primarily used to investigate the debris environment at high altitudes.

Results from five optical space debris surveys have been published so far: (1) LEO measurements from the NASA Liquid Mirror Telescope (LMT) (Africano et al. 1999), (2) GEO survey results from the so-called NASA CCD Debris Telescope (CDT) (Jarvis et al. 2002; Barker et al. 2005), (3) the NASA 


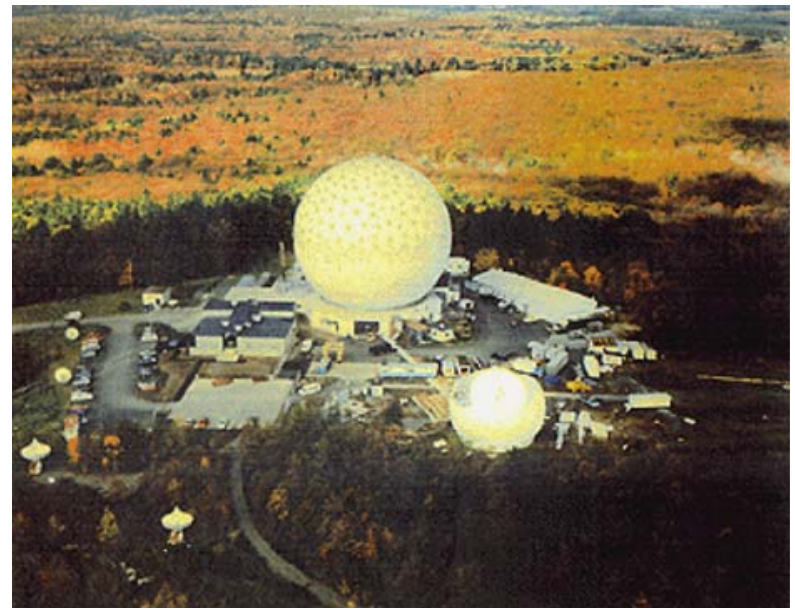

Fig. 10 Haystack radar complex. The 36-m parabolic dish antenna is located under the radome in the center of the figure

funded Michigan Orbital DEbris Survey Telescope (MODEST) (Seitzer et al. 2005), (4) the TAROT telescope supported by the French space agency CNES (Ríos Bergantiños et al. 2005), and (5) GEO and GTO observations from the ESA 1-m telescope (Schildknecht et al. 2004).

Liquid mirror telescope (LMT) Although optical sensors are typically used for high-altitude observations there is a prominent exception, the NASA 3-m liquid mirror telescope (LMT) located at Cloudcroft, New Mexico. The primary mirror of this telescope consists of a parabolic dish of spinning mercury, a setup which is much cheaper than a conventional glass mirror: The LMT was built for about a tenth of the cost of a traditional telescope with similar size. One disadvantage, however, is the inability to point the telescope to any other direction than the zenith. The LMT was used by NASA from 1996 to 1999 to characterize the LEO debris environment in the important but hard to track one-to-ten centimeter range. Results from the LMT included objects that were as small as $3 \mathrm{~cm}$. These optical observations are unique in the sense that they cover the same altitude and size range as the LEO debris radars and therefore provide independent and complementary statistical information, which can be compared with the radar results. Such comparisons allowed estimating the optical reflectivity of the debris pieces, which turned out to be of the order of $10 \%$, a value consistent with previous estimates. Overall, the LMT data are in fairly good agreement with the radar observations although the LMT fluxes are slightly higher than the corresponding radar results for altitudes below 1,000 km (Africano et al. 1999).

CCD debris telescope (CDT) The NASA CDT telescope is a small portable instrument with an aperture of $32 \mathrm{~cm}$ and a field of view of $1.7^{\circ}$ diameter. The instrument was used for test observations and an initial, limited, GEO survey 
from 1992 to 1995 on the Hawaiian island of Maui (Talent et al. 1997). From 1997 to 2000 NASA operated the instrument at Cloudcroft New Mexico during more than 140 nights and thereby conducted the first extensive space debris survey in GEO. Considering the rather small aperture of this instrument the survey could 'only' detect objects as small as about $30-40 \mathrm{~cm}$ at a distance of $36,000 \mathrm{~km}$. From these measurements it was immediately concluded that the limiting size of the USSTRATCOM catalog for GEO is indeed at about $1 \mathrm{~m}$, and more important that there is also considerable population of smaller objects not covered by the catalog. Moreover, it is also suspected that the catalog is not complete for object sizes larger than $1 \mathrm{~m}$. At this point we may mention that the public version of the USSTRATCOM catalog is intentionally leaving out about 123 classified GEO objects (status 2002) - this fact was taken into account when assessing the completeness of the catalog.

Optical observations primarily measure the apparent brightness of an object expressed in astronomical magnitudes. This quantity is, of course, depending on the distance between the object and the observer and on the illumination conditions. If the observation geometry (Sun-observer-object) and the distance to the object at the moment of the measurement are known, the apparent magnitudes may be converted to the so-called absolute magnitudes which are referred to a conventional distance (the nominal GEO distance) and to a standard illumination condition (Sun on the line object-observer, behind the observer). (We have to admit that some light scattering properties of the objects have to be adopted in order to perform this transformation.) Finally, object diameters may be derived from absolute magnitudes by making some reasonable assumptions on the reflectivity (albedo) and the shape of the considered objects.

The conversion of the measured brightness of an object to its physical size is a notoriously difficult undertaking. This is not surprising because the threedimensional shape of the object as well as the scattering properties and the albedo of its surfaces are usually unknown for space debris! Ideally, we would require laboratory experiments, e.g., analyzing the properties of explosion fragments of real space hardware, like it was done for radar (Bohannon and Young 1993). So far no experiments have been performed to study the optical properties of debris and we are thus forced to make some reasonable assumptions. For the shape we assume either a perfect sphere or a randomly tumbling plate, both having Lambertian scattering properties. The geometric albedo of large known GEO objects has been estimated by Henize and Stanley (1990), which derived an astonishingly small value of 0.08 with a large scatter of 0.35 in log albedo. Note that the value for debris pieces could considerably differ from the value for large, intact objects. Optical measurements in LEO are currently conducted by NASA trying to derive the albedo of known small-size debris by comparing the optical brightness with the corresponding diameters derived by radar (Kessler and Jarvis 2004; Africano et al. 2004). First results indicate a mean albedo value between 0.1 and 0.2 . The estimate is, of course, based on assumptions concerning the shape and scattering properties.

Figure 11 shows the histogram of the absolute magnitudes of the objects observed in the CDT GEO survey during 25 nights from March to May 1998. 


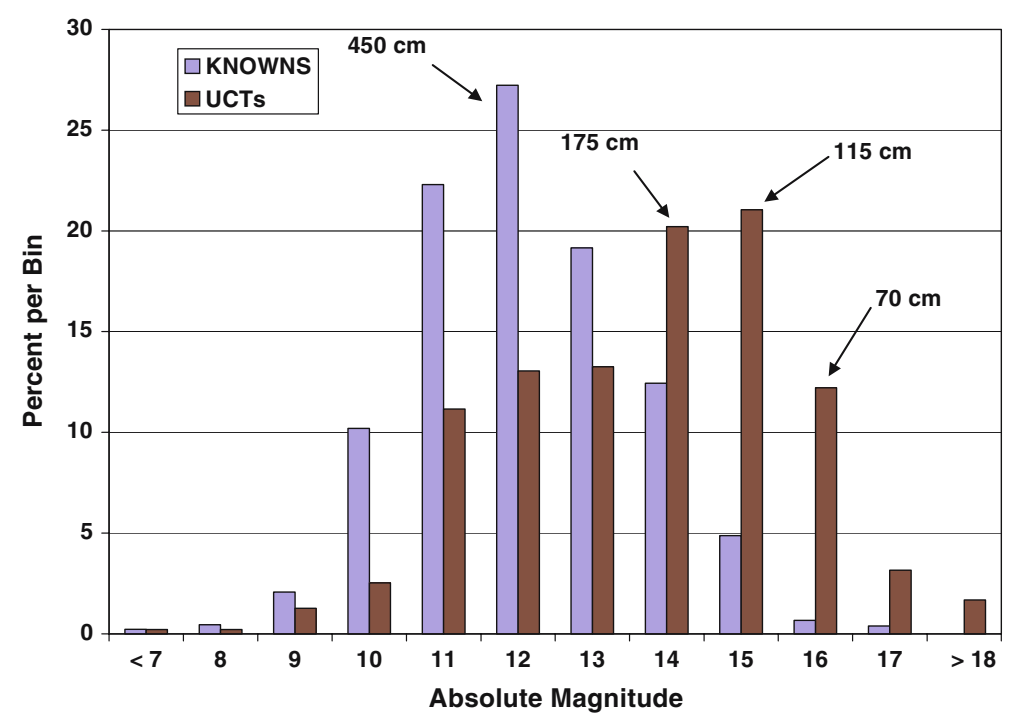

Fig. 11 Distribution of absolute magnitudes from the NASA CDT GEO survey (from Africano and Schildknecht 2000)

The terms 'knowns' and 'UCTs' ('uncorrelated targets') refer to the objects, which could or could not be identified in the catalog. The indicated object sizes were derived using an assumed reflectivity of $20 \%$ for the objects. The figure clearly illustrates the mentioned unknown population of small objects as well as the potential incompleteness of the catalog at larger sizes.

Michigan orbital debris survey telescope (MODEST) The Michigan orbital debris survey telescope is a $0.61 / 0.91-\mathrm{m} \mathrm{Schmidt} \mathrm{telescope} \mathrm{belonging} \mathrm{to} \mathrm{the} \mathrm{Univer-}$ sity of Michigan and located at the Cerro Tololo Inter-American Observatory in Chile. The system is performing surveys for space debris in GEO during several weeks per year since February 2001. Most of the observations are acquired in the context of coordinated observing campaigns organized by the Inter-Agency Space Debris Coordination Committee (IADC) (see Sect. 2.4.2).

MODEST is equipped with a 2,048 $\times 2,048$ pixel, thinned, backside illuminated CCD covering a field of view of $1.3^{\circ} \times 1.3^{\circ}$, with 2.3 arcsec pixels. The limiting magnitude of this sensor is about 17.5 in the Cousins R-band. Figure 12 shows the distribution of observed $\mathrm{R}$ magnitudes for all non-station keeping objects found during one campaign (from Seitzer et al. 2005). Station keeping objects defined as those within $0.5^{\circ}$ of zero declination and with a total observed angular motion less than $0.01 \mathrm{arcsec} / \mathrm{second}$ have been excluded from this sample. The MODEST data has not been correlated with a catalog, which means that we cannot distinguish between 'known' and 'unknown' debris. The distribution is bimodal with a first maximum at about magnitude 12 and a second peak at magnitude 17. The first maximum coincides with the peak of the distribution of the 'known' objects as observed by CDT (see Fig. 11). The second maximum 


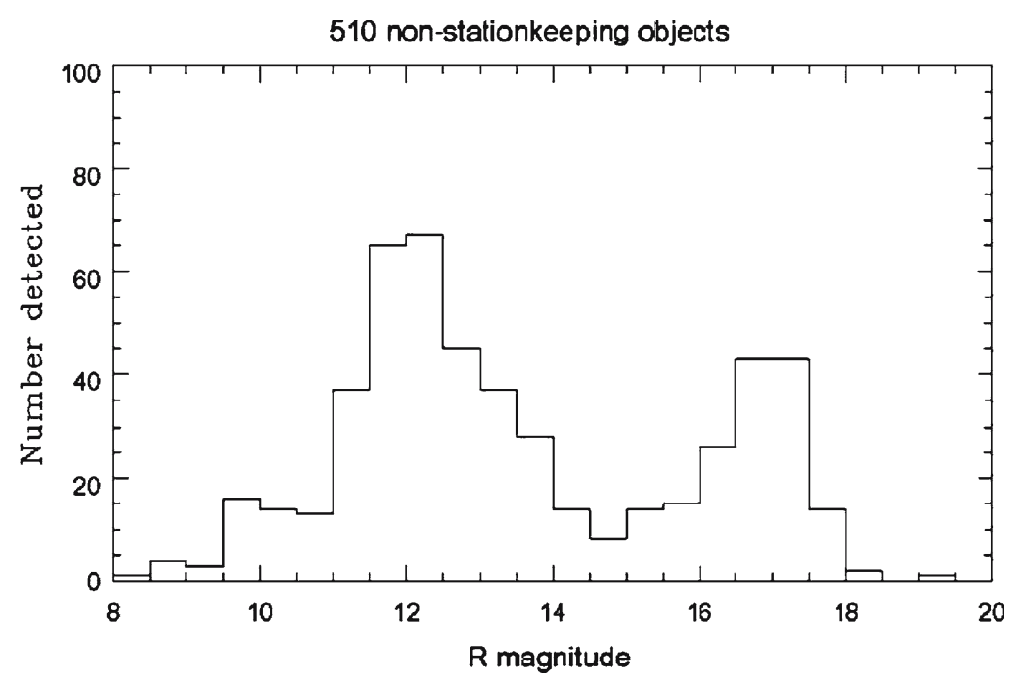

Fig. 12 Distribution of observed R magnitudes for all non-station keeping objects found during one MODEST campaign (from Seitzer et al. 2005)

is obviously not present in the CDT data because the limiting magnitude of the CDT is about 16. The fall-off in the MODEST distribution at magnitudes fainter than magnitude 17.5 is caused by the decline in sensitivity of the survey.

TAROT The French National Space Agency (CNES) uses the Rapid Action Telescope for Transient Objects (TAROT) to participate in the IADC GEO surveys. The $0.25-\mathrm{m}$ telescope TAROT is installed on the Calern plateau above Grasse in the South of France and belongs to the National Scientific Research Centre (CNRS). Its primary goal is the observation of optical counterparts of gamma ray burst. The instrumentation consists of an 2,048 $\times 2,048$ pixel backside illuminated CCD with a field of view of $1.85^{\circ} \times 1.85^{\circ}$ and a pixel size of 3.2 arcsec. Data with this sensor was collected during the 2004 IADC GEO campaign, which lasted 4 weeks in total.

The distribution of observed magnitudes from the 18 nights of the 2004 campaign is given in Fig. 13 (from Ríos Bergantiños et al. 2005). Detections, which could be identified with an object in the USSTRATCOM catalog, are labeled 'GEO-CTs' or 'GTO-CTs' and 'unknown' objects are labeled 'GEO-UCTs' or 'GTO-UCTs'. (The distinction between GEO and GTO is not based on 6-parameter orbits and is misleading, as the majority of the detected object are most probably GEO objects.) The maximum of the distribution of the 'known' objects is at about magnitude 10.5 , which is more than one magnitude less than for the CDT and the MODEST surveys. This is actually the first TAROT space debris data and the magnitudes, although calibrated internally, have not yet been cross calibrated with data from other sensors. The distributions for both, the 'known' and the 'unknown' objects, fall off at magnitudes fainter than 14 because of the limiting magnitude of the system, which is about 14.5 . 


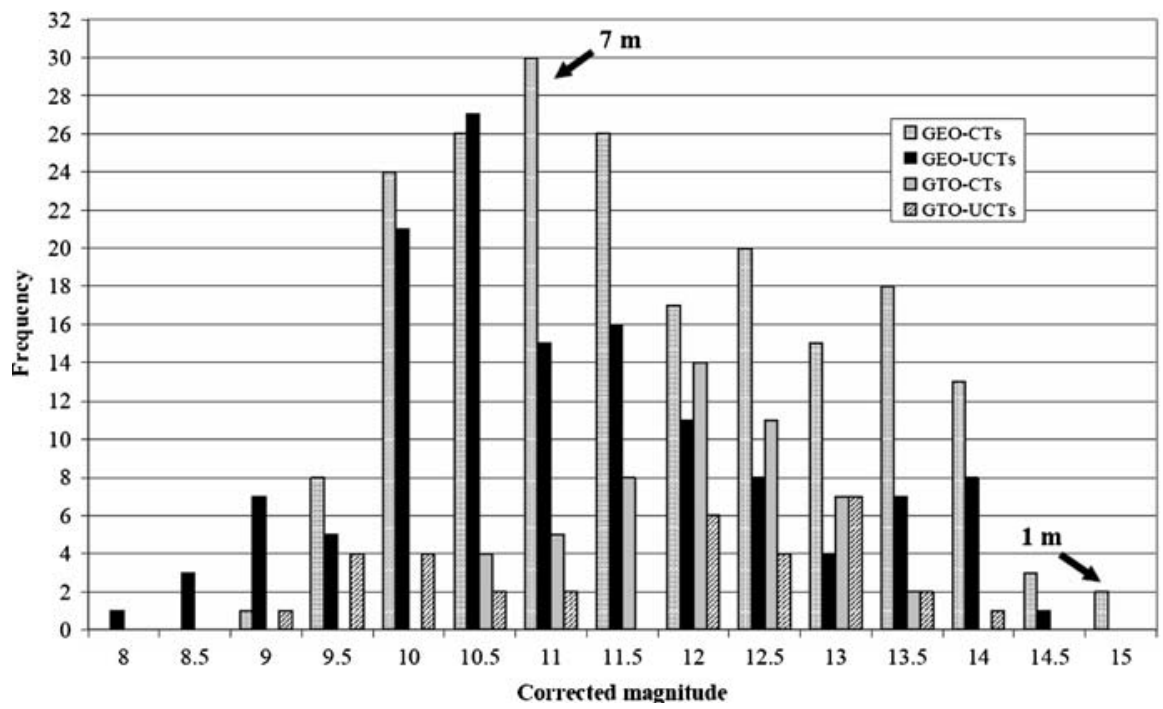

Fig. 13 Distribution of observed magnitudes of the 2004 TAROT data (from Ríos Bergantiños et al. 2005)

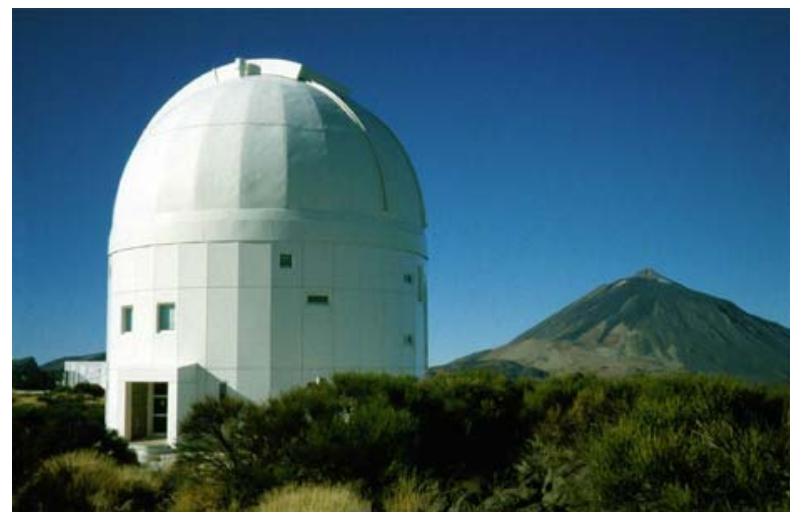

Fig. 14 Dome of the ESA Space Debris Observation Telescope at the Teide Observatory on Tenerife, Canary Islands. The Teide volcano seen in the background is the highest peak of Spain $(3,715 \mathrm{~m})$ and the third largest volcano on Earth (after Mauna Loa and Mauna Kea)

ESA 1-meter telescope ESA started an optical GEO survey program, which became operational in 1999. The program is using ESA's 1-m telescope in Tenerife, Canary Islands. Figure 14 shows the dome of the ESA telescope located at an altitude of $2,400 \mathrm{~m}$ about $20 \mathrm{~km}$ northeast of the Teide volcano which can be seen in the background. The ESA 1-m telescope is installed in the Optical Ground Station (OGS) which was originally established in the framework of the data relay and technology mission for the orbit check-out of the optical communication payload of the ARTEMIS spacecraft. Afterwards, an upgrade of the telescope for space debris observations was realized. The instrumentation 


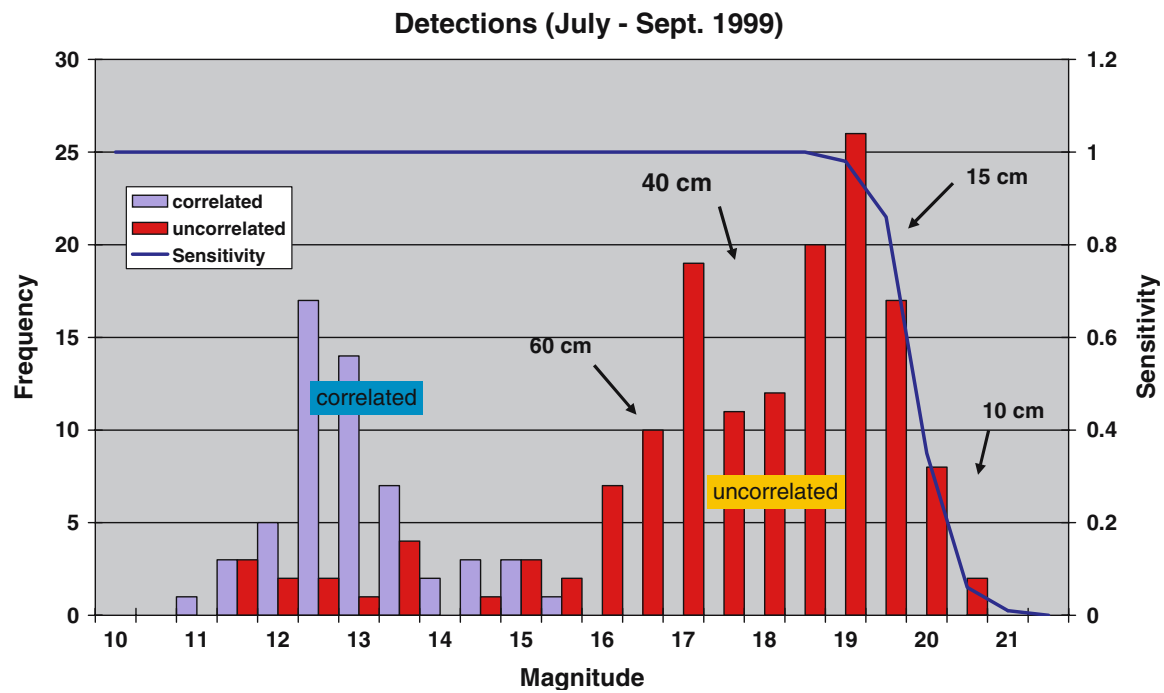

Fig. 15 Distribution of absolute magnitudes from the ESA 1999 GEO survey. The solid line shows the instrument sensitivity as determined from independent calibration observations (from Schildknecht et al. 2004)

includes a dedicated cryogenically cooled space debris camera consisting of a mosaic of CCD detectors with a total of 4,096 $\times 4,096$ pixels. The field of view of this camera is $0.7^{\circ} \times 0.7^{\circ}$ and a single pixel corresponds to 0.6 arcsec.

The Astronomical Institute of the University of Bern (AIUB) is very actively involved in this program. The AIUB has been developing software systems for the observation planning, the actual data acquisition at the telescope and the on- and off-line data processing. The AIUB is also performing the operational GEO survey for ESA.

Compared to the CDT, the MODEST and the TAROT systems the field of view of this telescope is considerably smaller. But due to the larger aperture the instrument can detect objects as faint as magnitude 20 corresponding to diameters as small as $10-15 \mathrm{~cm}$ in the GEO.

Figure 15 shows the absolute magnitude diagram from a very limited ESA GEO survey campaign in July and September 1999 (13 nights only). The solid line shows the instrument sensitivity as determined from independent calibration observations. The indicated object sizes were derived by assuming Lambertian spheres and an albedo of 0.1. The distribution is bimodal with the cataloged (correlated) objects clustered around magnitude 12.5, and a large population of uncataloged (uncorrelated) objects in the range from magnitude 15 to 21 . It is important to note that the decrease in the number of objects fainter than magnitude 18 is entirely due to the limiting magnitude of the observation system. The real number of objects fainter than magnitude 18 could therefore still increase! These observations actually discovered a hitherto unknown significant population of uncataloged small-size debris objects in the $10-100 \mathrm{~cm}$ size range in the GEO. 


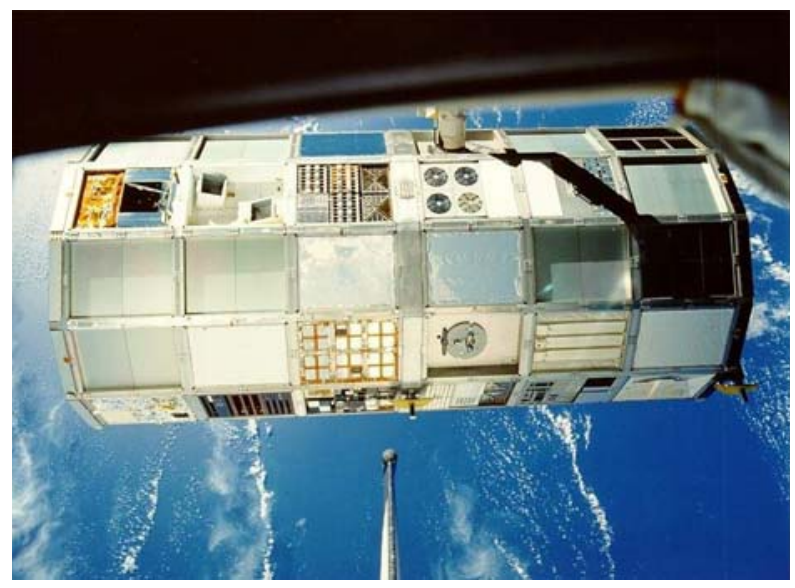

Fig. 16 Long duration exposure facility (LDEF) during the retrieval by the US space shuttle

\subsubsection{In situ measurements}

Much information on multimeter and sub-multimeter-sized particles may by obtained from the analysis of surfaces exposed to the debris and micrometeorite environment after retrieving them from space. Such surfaces may be available either on retrieved spacecraft or parts thereof or in the form of dedicated passive dust detectors brought back to Earth. Passive detectors usually contain a surface, which is designed to record impacts of small particles; some detectors may also catch the impactors for further analysis of their composition. Surfaces retrieved from space are covered with a large number of impact craters. For a given material the size and shape of these craters may be related to the sizes of the impacting objects using sophisticated computer models and ground calibration tests (hypervelocity impact test). From these number counts in a known orbit, with known attitude and exposure time for the surface of interest finally statistical particle fluxes as a function of particle size may be derived. The degree of information on the orbits of the impacting particles varies considerably depending on the setup of the experiment (many of the analyzed surfaces were not primarily designed as particle detectors).

The long duration exposure facility (LDEF) is the most prominent example of a dedicated experiment (Fig. 16). The structure has the size of a bus and was retrieved in January 1990 by a space shuttle after having been exposed for 5.6 years to the space environment. More than 34,000 impacts were visible to the naked eye and the largest crater had a diameter of $5 \mathrm{~mm}$. The analysis of the LDEF experiments indicated that impacts were clustered in time, an evidence for particle streams or clusters.

In principle, any piece of hardware retrieved from space may be analyzed for particle impacts. Figure 17 shows impact craters on solar arrays retrieved from space. Part of the solar array of the European retrievable carrier EUREKA is shown on the left-hand side, on the right-hand side a hole in the solar panel 

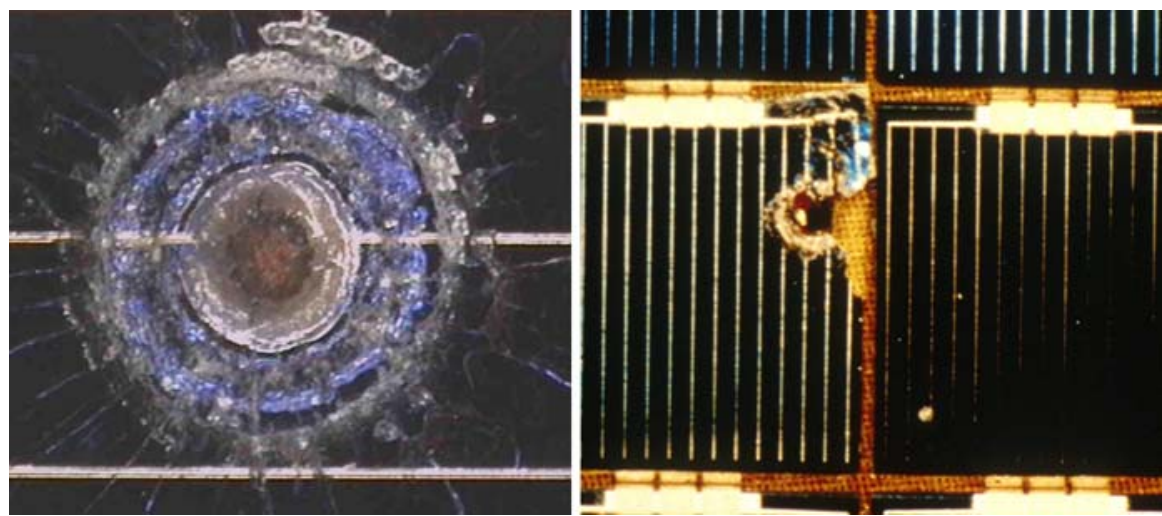

Fig. 17 Impact craters on solar arrays retrieved from space. Part of the solar array of the European retrievable carrier EUREKA on the left-hand side, on the right-hand side a hole in the Hubble Space Telescope solar panel (the vertical lines are spaced by $1.2 \mathrm{~mm}$ ) (from ESA)

of the Hubble Space Telescope can be seen (the vertical lines are spaced by $1.2 \mathrm{~mm})$.

A major difficulty of in situ measurements is the discrimination between impact from natural micrometeorites and space debris particles. The two sources can only be distinguished by a chemical analysis of residues of the impactor. Due to the high impact velocities, however, the particle in many cases totally evaporates or its material is mixed with the material of the penetrated surface. Figure 18 gives the debris flux as a function of the object size in LEO as measured by different experiments. All measurements stem from in situ measurements, except for the 'Haystack' and 'HAX', which are radar measurements, and the 'TLE', which is the USSTRATCOM data. For sizes below 10 microns and above about $1 \mathrm{~cm}$ the environment is dominated by space debris while in between the debris flux is about the same as the micrometeorite flux.

\subsubsection{Models}

Models are used to describe the space debris population mathematically. Models are a prerequisite to perform risk analysis studies for individual spacecrafts. Nowadays every space mission, starting from its design, is analyzed for this risk and protective measures are taken, either by shielding critical components or by choosing an optimized orbit. Space debris environment models are, of course, all based on measurements but they differ in their approach to represent the observations. Purely empirical models fit the observed fluxes with an appropriate mathematical functions while deterministic models try to reproduce the observations with physical models starting from the known debris sources like the launches, explosions, etc. Both approaches describe the current measurements equally well, but deterministic models offer more insight into the physical mechanisms leading to the production and proliferation of space debris. Figure 19 shows the spatial density of debris objects larger than $10 \mathrm{~cm}$ as given 


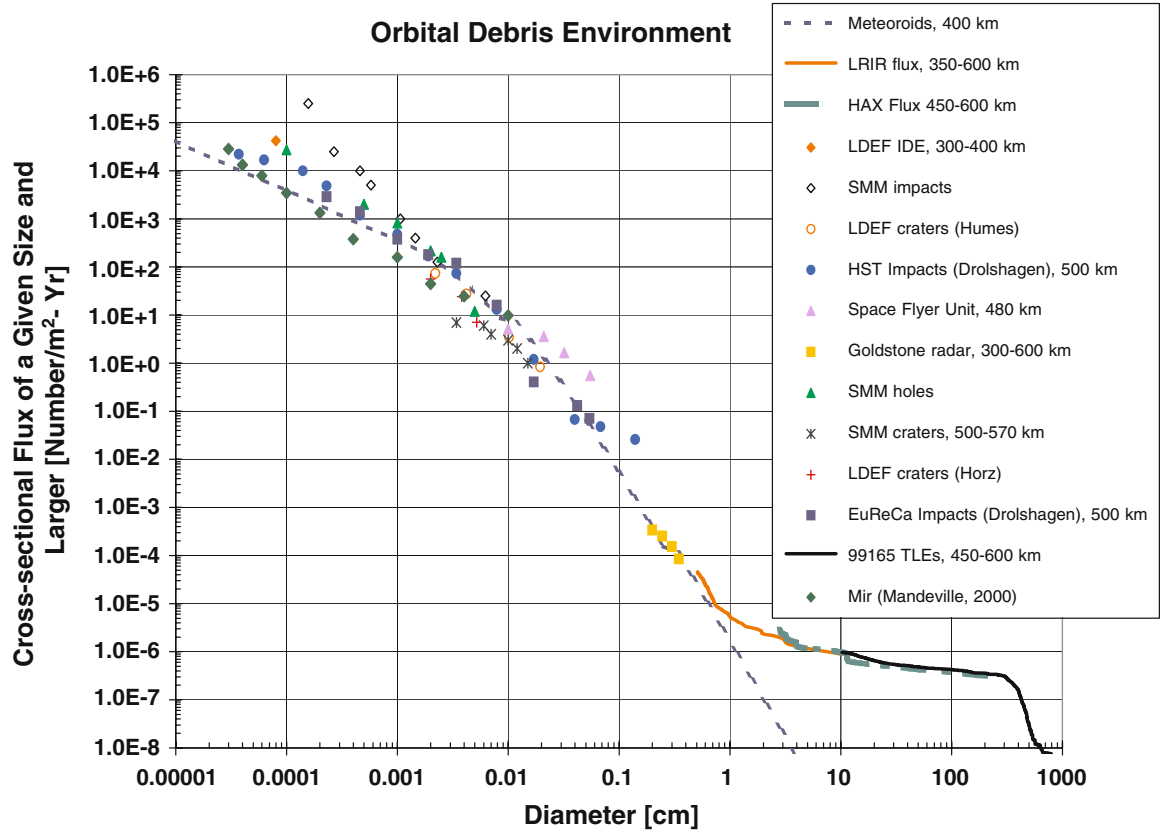

Fig. 18 Measured space debris flux in LEO by object size (from IAA position paper on orbital debris 2001)

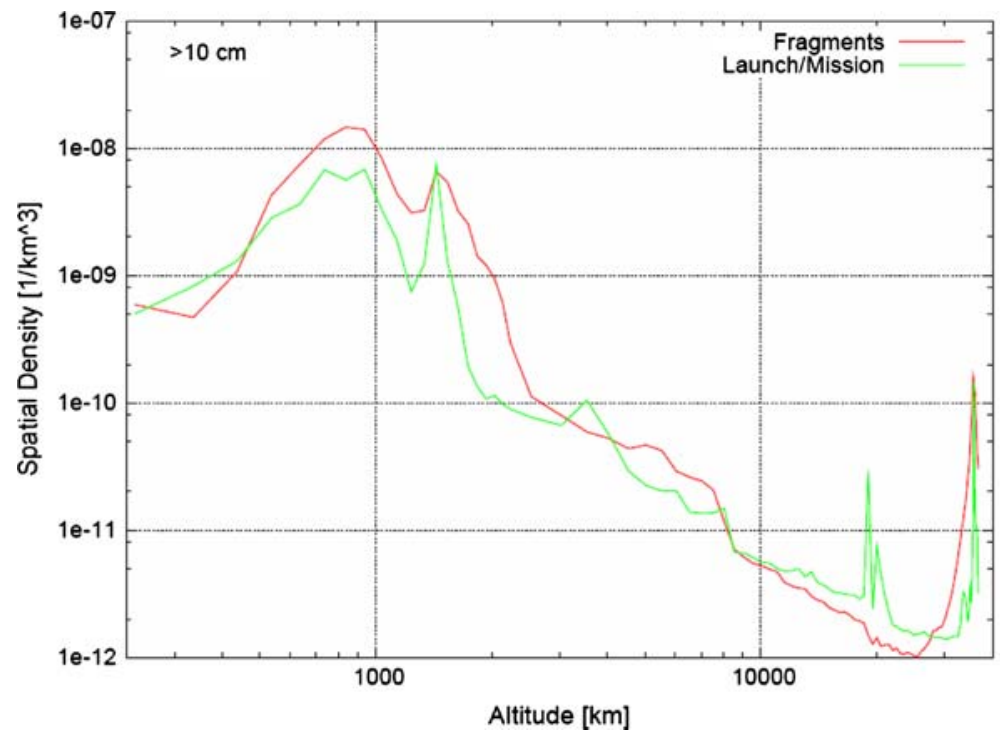

Fig. 19 Spatial density of debris objects larger than $10 \mathrm{~cm}$ as given by the ESA MASTER 2001 model ('Fragments'). Densities for the USSTRATCOM catalog ('Launch/Mission') are also given for comparison (from Bendisch et al. 2002) 
by the ESA MASTER 2001 model ('Fragments') (Bendisch et al. 2002, 2004). Densities for the USSTRATCOM catalog ('Launch/Mission') are also given for comparison. The highest density occurs at altitudes around $800-1000 \mathrm{~km}$ both in the catalog and in the debris population. Strong peaks in the catalog population are also visible at 20,0000 and $36,000 \mathrm{~km}$ altitude, the region of the 12 -h orbits of the US and Russian navigation systems (GPS and GLONASS) and the GEO region respectively. The fragment population at the GEO altitude is much too small in this model compared with results from recent optical GEO surveys. This indicates that the GEO surveys substantially improved our knowledge of the debris population in the GEO.

Models are also necessary to study the future evolution of the debris environment and the effect of mitigation measures. The driving quantities of evolution models are the assumptions about the future evolution of the primary debris sources like the number of launches, related satellites and orbits, the number of explosions, etc. Another important mechanism for debris generation is the collision of objects. Each collision produces many new fragments, which further enhance the density of the environment. Above a so-called critical density, which depends on the source and sink rates at the given altitude, a cascading of collisions takes place, leading to an exponential growth of the population. It is worth noting that at this point even terminating all launch activities will not prevent the population from growing further! Currently, most evolution models predict that without mitigation measures the collisional fragments will become the main debris source in a few decades. Figure 20 shows the number of major collisions for three scenarios as predicted from a number of different models. The 'business as usual' case assumed a normal rate for launches (no substantial growth!) and explosions but no mitigation measures. With this scenario we have to expect about one major collision per year by the end of the 21 st century.

\subsection{Protecting the space environment}

In the previous sections we have shown that potential collisions with space debris are a real hazard for all space operations in the near-Earth space. (For the sake of this discussion we define the term 'near-Earth space' as the region were objects are gravitationally bound to the Earth, in particular including LEO, GTO and GEO.) The current threat posed to spacecraft is in most orbital regions still rather low-only one confirmed collision between an operational spacecraft and a debris piece occurred until today. However, the risk for longterm missions is already significant in LEO. For the international space station ISS, for example, we must expect at least one impact penetrating one of its pressurized modules over its lifetime even taking into account the sophisticated protection shields of the station. Most critical, however, is the future evolution of the space debris environment. Current evolutionary models do not fully agree quantitatively due to slightly different assumptions and limitations of the models. But all investigations conclude that qualitatively the space debris population in certain orbital regions will become so dense that space operations 


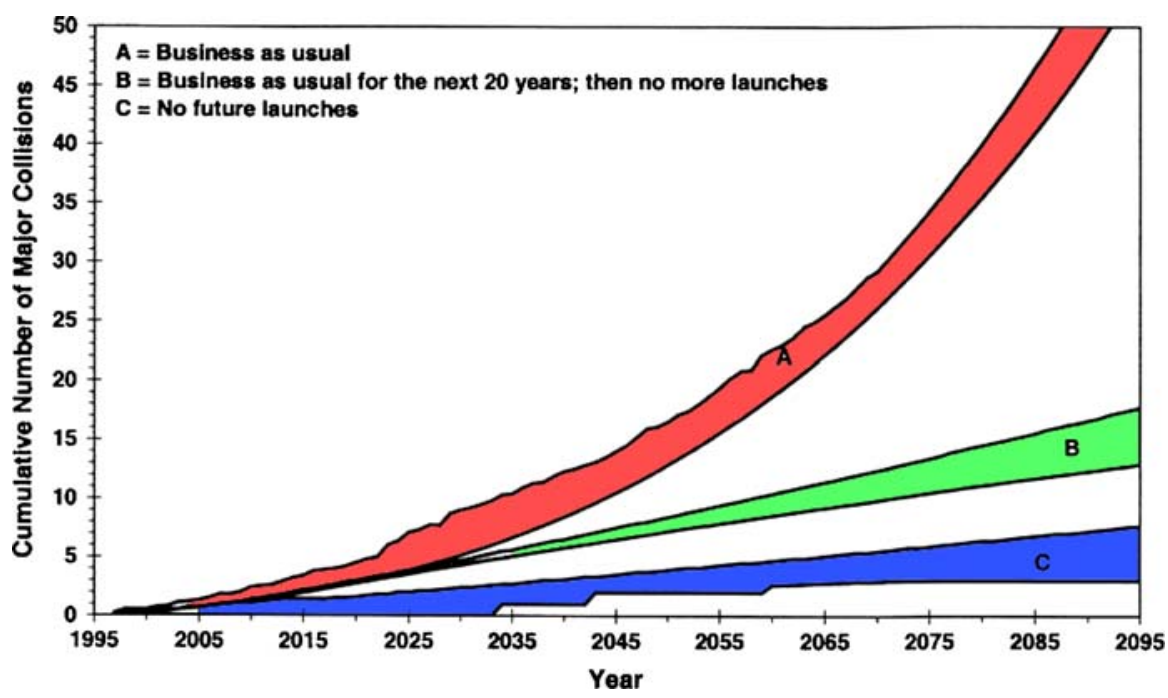

Fig. 20 Typical ranges for the number of major collisions for three scenarios (from IAA position paper on orbital debris 2001)

will become severely hampered after a few decades, if space operations are continued to be conducted in the same manner as today. This conclusion can be drawn without even assuming an increased launch rate in future and without considering large constellations of hundreds of satellites as planned a few years ago by several communication industry companies.

On the other hand, we realize that space cannot be cleaned from debris with any technical means in near future. Many options have been studied in recent years, including, e.g., tethers to de-orbit larger objects or ground-based high power lasers to perturb the orbits of small fragment forcing the fragments to reenter the atmosphere. All proposed techniques are either not feasible in the coming decades or they are prohibitively expensive. It is therefore obvious that the only viable way to allow future generations to conduct space operations in near-Earth space, and especially in unique orbital regions like GEO, is to protect this environment by severely limiting the production of space debris.

\subsubsection{Space debris mitigation}

Currently, the vast majority of debris larger than a centimeter stems from fragmentations. For the cataloged objects, i.e., objects larger than about $20 \mathrm{~cm}$ in size, fragments account for $40 \%$ of the population. Except for microparticles smaller than a few millimeters explosion fragments are thus the main source of space debris. Mutual collisions among these fragments will produce a new population of collisional fragments, which may eventually lead to an exponential growth of the entire population. The prevention of in-orbit explosions is therefore by far the most effective debris mitigation measure! 
In order to prevent explosions all space hardware should be passivated after the end of its useful live. Passivation means the removal of all stored energy like residual fuel in rocket upper stages and attitude control system or energy stored in batteries. Several techniques like the venting of residual fuel or so-called depletion burns are already applied in several launchers. The measures are cost-effective and do not require new technologies to be developed.

Mission related objects like protective instrument covers, explosive bolts from separation mechanisms, adapter rings and structures required for multi satellite launches, etc. are another important category of space debris. Technically, the release of such objects may in most cases, except for the large structures, be prevented by implementing rather simple mechanisms to retain the objects. These are again cost-effective measures, provided that they are considered during the design of the vehicles.

In densely populated regions this may not be sufficient and spacecrafts will have to be actively removed. Ideally, all hardware should be brought back to Earth (this procedure is also called 'de-orbiting'). Depending on the altitude of the orbit, this requirement would have a severe cost impact! In LEO one may take advantage of the air drag, which eventually forces all orbiting objects to reenter the atmosphere. For objects in altitudes below $600 \mathrm{~km}$ the natural decay time for their orbits due to air drag is a few years at maximum and thus no additional measures are required. Decay times for higher LEO orbits are of the order of many decades or even centuries. The International Academy of Astronautics (IAA) has thus adopted a rule which states that the orbital lifetime for spent upper stages and defunct spacecraft in orbits with a perigee below 2,000 km should be limited to 25 years (IAA position paper on orbital debris 2001). Note that this rule includes most objects on GTO orbits! In order to comply with this rule new technical developments will be required and their cost-effectiveness be demonstrated. Options to actively accelerate the orbital decay are, e.g., the use of on-board propulsion systems, additional dedicated maneuvering vehicles and drag enhancement devices. The use of the latter is a questionable technique because of their large cross section, which increases the collision probability.

The costs to de-orbit objects from orbits with perigees above $2,000 \mathrm{~km}$, in particular from the GEO region, are substantial or even prohibitively high. The IAA rule therefore requires for these regimes that objects are reorbited at the end of their mission into disposal (also called 'graveyard') orbits as a temporary measure. Several international organizations have established concrete rules for GEO which all define a disposal region several hundred kilometers above the GEO. In practice the reorbit maneuver requires about the same amount of fuel as needed to keep a satellite a few months at its position (also called 'station keeping'). The critical aspect of the requirement is the fact that satellite operators have to initiate the reorbiting before the actual end of live of the spacecraft and must make sure that there is still enough fuel available for the maneuver - a decision with a direct economic impact for the operator. During the year 2002 only 5 of the 13 GEO satellites, which reached their end of life, were properly reobited into a disposal orbit (Hernández and Jehn 2003)! 


\subsubsection{International cooperation}

Space debris mitigation cannot be achieved 'for free'. Even the cost-effective measures to prevent explosions may not be implemented in view of the strong economic competition in the market. Moreover, the topic touches sensitive policy aspects like economical and national security aspects. It is therefore obvious that only international cooperative actions of all space faring nations might eventually lead to an effective protection of near-Earth space. Apart from the technical approaches mentioned above, legal aspects must finally be addressed, as well.

An important international body addressing technical issues related to space debris is the Inter-Agency Space Debris Coordination Committee (IADC). IADC currently has 11 members including all major space agencies. The four working groups - measurements, modeling, protection and mitigation-initiated substantial research activities in all four fields. Results from these studies have led to IADC recommendations and documents, which although not legally binding, have been adopted by all members and were at least partially included into national standards and regulations. Contributions from the IADC were substantial, both for IAA position paper on orbital debris (2001) and for the UN technical report on space debris (1999). The author of this review is an ESA delegate in the IADC working group for measurements and is coordinating some of the optical measurement activities (Africano and Schildknecht 2000, 2003, 2004).

In 1999 the Scientific and Technical Subcommittee of the United Nations Committee on the Peaceful Uses of Outer Space (UNCOPUOS) endorsed the UN technical report on space debris. This was a major step after a multi-year review of the topic by the subcommittee. Eventually, the subject should be included into the agenda of the legal subcommittee.

\subsection{Summary}

Space operations inevitably produced debris since the beginning of the space age. The inventory of space debris ranges from large objects like defunct satellites and rocket upper stages over mid-size fragments of explosions to smallsized particles like paint flakes and solid rocket motor dust.

The hazard emanating from space debris concerns primarily space operations themselves. The risk due to objects decaying from orbit and reaching the ground is negligible, provided a controlled de-orbiting is performed for all risk objects with masses larger than five tons and provided no radioactive material is involved.

The current space debris population is comparatively well known for large objects and for microparticles in the LEO, but there is still a lack of information in the range between 1 and $20 \mathrm{~cm}$ in LEO and for sizes $<1 \mathrm{~m}$ in other orbital regimes. Observation of space debris with ground-based radar and optical facilities as well as through in situ measurements should therefore continue 
and in particular focus on the mentioned orbit and size regimes. A permanent monitoring is required in view of the dynamical nature of the population.

The present risk to spacecrafts in orbit by space debris is not yet critical, but future space operations will be severely hampered by space debris in a few decades if no substantial debris mitigation measures are implemented.

There is no technically feasible method to clean space from debris. Debris mitigation measures must be internationally agreed in a binding form and implemented in a consistent manner. They should at least include the prevention of all in-orbit explosions by passivation of all defunct hardware in space. As passivation measures are not sufficient to avoid an unacceptable growth of the population, objects in LEO orbits should be actively de-orbited or decay after a maximum of 25 years. Objects in GEO should be consistently placed into disposal orbits at the end of their mission.

Currently, the growth of the space debris population is not yet out of control, but actions to substantially mitigate space debris in future are urgently needed. This is the only chance we have to preserve near-Earth space in a way that future generations may continue to perform space operations in a technically and economically feasible way.

\section{Observation techniques}

\subsection{Characteristics of objects in high-altitude earth orbits}

Conventionally, orbits with apogees at altitudes higher than 2,000 km are called 'high-altitude' Earth orbits. There exist a variety of different orbit families, which belong to this class. The most well known family consists of the so-called GEO at 36,000 km altitude. Objects in such orbits are more or less stationary in an Earth fixed frame. The GEO region is extensively used for communication satellites (data relay, TV broadcast, etc.). Objects are placed in GEO by using the so-called GTOs, which are highly elliptical orbits with apogees near the GEO altitude and perigees located at low altitudes of 300-800 km. Objects remaining in GTO are mainly intact spent upper stages and mission related objects like payload adapters, or explosion debris thereof.

Global navigation satellite systems like GPS, GLONASS and GALILEO are placed in orbits with altitudes between 20,000 and $22,000 \mathrm{~km}$ resulting in orbital periods of approximately $12-14 \mathrm{~h}$. This orbital region is often called medium Earth orbit (MEO) region.

A large series of Russian communication satellites is using highly elliptical orbits with periods of $12 \mathrm{~h}$. The perigees of these so-called Molniya orbits are located at $400-600 \mathrm{~km}$ in the southern hemisphere and the apogees are at altitudes of $40,000 \mathrm{~km}$ above the northern hemisphere. This family of orbits is often subsumed together with other highly eccentric orbits under the term 'high eccentric Earth orbits' (HEO).

Most optical space debris surveys are currently concentrating their efforts to investigate the debris population in GEO. The only exception is the ESA 
survey, which includes observations dedicated to detect debris in the GTO region. We therefore restrict the following discussion to these two regions.

\subsubsection{Basic characteristics of GEO orbits}

Ideally, geostationary objects are in circular orbits in the equatorial plane (i.e., with an inclination $i=0^{\circ}$ ) and a mean motion of one sidereal revolution per sidereal day. The radius of a perfect GEO orbit is $42,164 \mathrm{~km}$. In reality GEO spacecrafts are controlled to maintain their orbits within certain ranges. Direct broadcasting TV satellites, e.g., are usually kept within a so-called longitude slot of $0.1^{\circ}$ and their latitude is confined to $\pm 0.1^{\circ}$ by controlling the inclination of the orbit. In order to maintain its position a GEO spacecraft must regularly perform the so-called station-keeping maneuvers due to external accelerations perturbing its nominal orbit. The main forces perturbing orbits in GEO are the anomalies in the gravitational field of the Earth, the gravitational attraction of the Sun, and the Moon and the solar radiation pressure. Longitude-dependent spherical harmonics of the development of the gravitational potential of the Earth, in particular the term $J_{22}$, lead to resonance effects acting as long-term perturbations of the semimajor axis. The result is a librational motion of the objects around the nearest stable point either at $75^{\circ} \mathrm{E}$ or $105^{\circ} \mathrm{W}$. The oblateness of the Earth and the gravitational pull from the Sun and the Moon force the orbital planes to precess with a period of about 53 years around a stable plane (Allan and Cook 1964). This plane, the so-called Laplace plane, has an inclination of about $7.5^{\circ}$ with respect to the equatorial plane and its nodal line coincides with the direction of the vernal equinox (i.e., the normal of the Laplace plane is at right ascension $=270^{\circ}$ and declination $=82.5^{\circ}$ ). The corresponding variation in the inclination of the orbits is about $\pm 15^{\circ}$ (see Fig. 21). Solar radiation pressure, finally, induces a small eccentricity, leading to variations in the geocentric distance of up to $\pm 75 \mathrm{~km}$. The geostationary ring containing all longitude-controlled objects may thus be defined as a segment of a spherical shell centered in the equatorial plane with a radial thickness of $150 \mathrm{~km}$, delimited by $\pm 15^{\circ}$ in latitude (see Fig. 22). For objects with inclination control the width in latitude would be $0.2^{\circ}$. In the context of optical observations we may note that the characteristic resolution of 1 arc second corresponds to about $205 \mathrm{~m}$ at GEO as seen from Geocenter.

\subsubsection{Basic characteristics of GTO orbits}

A 'standard' GTO is usually defined as a highly elliptical orbit with its perigee located in the LEO region-defined as the region below 2,000 km altitude - and its apogee altitude equal to that of the GEO region being defined as $35,786 \pm 75 \mathrm{~km}$. Such orbits are used to transfer spacecrafts from LEO to GEO via various maneuvers. This is not a strict definition of the GTO region, but just the description of this orbit type at the moment when such transfers take place. Objects left in GTO are, however, subject to various perturbations. In particular their apogees will gradually be lowered due to atmospheric drag 


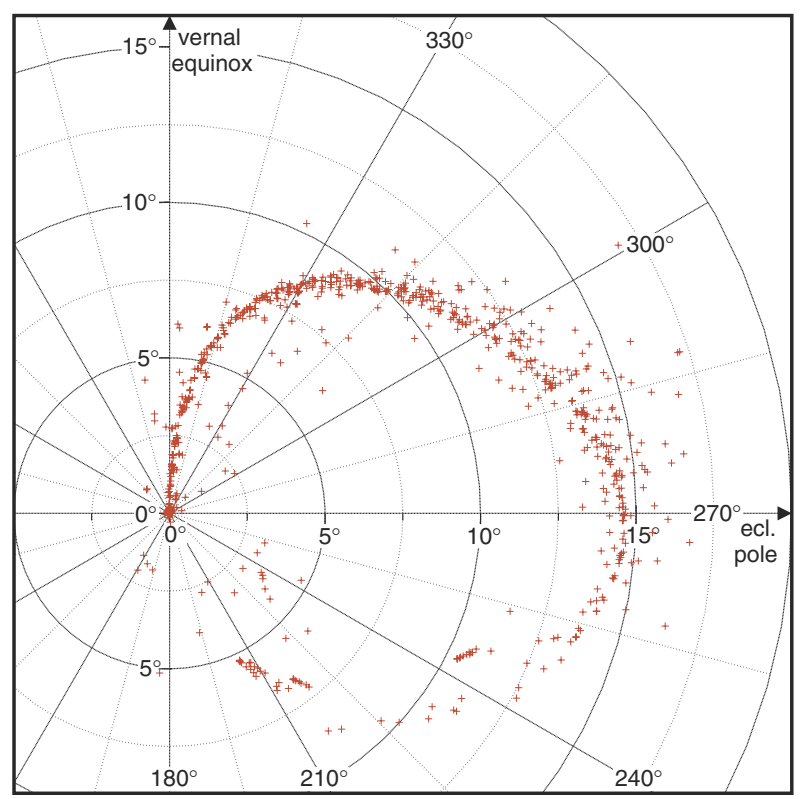

Fig. 21 Precession of the orbits of geostationary satellites. The symbols give the location of the orbital poles corresponding to inclination $i$ and right ascension of ascending node $\Omega$ of the cataloged objects (April 2006). Coordinates are right ascension $\alpha=\Omega-90^{\circ}$ and declination $\delta=i$

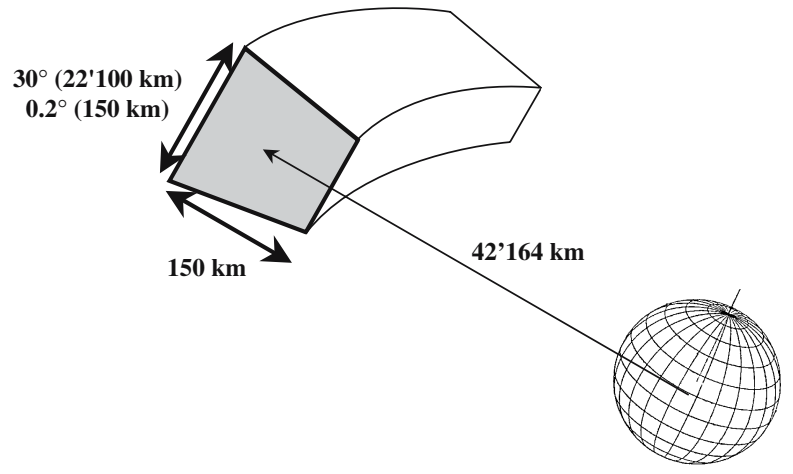

Fig. 22 The geostationary ring. The thickness in latitude is given for the case of objects with $\left( \pm 0.1^{\circ}\right)$ and without $\left( \pm 15^{\circ}\right)$ inclination control respectively

forces encountered during the perigee passages. As a result their orbits will be gradually 'circularized,' i.e., the height of their perigees will stay about constant, but their eccentricities will be lowered until the orbits become circular. For our purpose we will define the GTO region in a way that it includes objects which were originally on a 'standard' GTO but have decayed significantly in the meantime.

Table 2 shows the characteristics of a so-called "standard" geostationary transfer orbit for a direct transfer from LEO to GEO. The nominal parameters 
Table 2 Characteristics of the 'standard' GTO

\begin{tabular}{llll}
\hline Orbital parameter & Standard values & Ariane 4 & Ariane 5 \\
\hline Semi-major axis, $a(\mathrm{~km})$ & 24,370 & 24,364 & 24,596 \\
Eccentricity, $e$ & 0.73 & 0.73 & 0.718 \\
Inclination, $i(\mathrm{deg})$ & Inclination depends on launch site & 7 & 7 \\
Argument of perigee, $\omega(\mathrm{deg})$ & Depends on launch & 178 & 178 \\
Apogee altitude, $h_{a}(\mathrm{~km})$ & 35,786 & 35,786 & 35,890 \\
Perigee altitude, $h_{p}(\mathrm{~km})$ & 185 & 200 & 560 \\
Orbital Period, $T$ & $10 \mathrm{~h} 30 \mathrm{~m} 46 \mathrm{~s}=37,846.45 \mathrm{~s}$ & $37,847 \mathrm{~s}$ & $38,400 \mathrm{~s}$ \\
Mean orbital motion, $n($ rev/day) & 2.2829 & 2.28 & 2.25 \\
\hline
\end{tabular}

of a GTO for Ariane 4 and Ariane 5 are also included (Ariane 4 User's Manual 1992; Ariane 5 User's Manual 2000). These parameters are presented as a guideline only, as they may vary greatly from those for actual GTO objects.

Due to the Earth's oblateness the orbital plane of a 'standard' GTO precesses causing the ascending node to move backwards at a rate of about $0.4^{\circ} /$ day. In addition, the argument of perigee moves prograde at a rate of approximately $0.8^{\circ}$ day. As a result the longitude of the apogee moves at a rate of about $0.4^{\circ}$ /day accumulating to about $145^{\circ}$ year. This rate even increases with the decay of the semimajor axis due to air drag forces at the perigee.

The semimajor axes $a$ and the eccentricities $e$ of decaying GTOs are related due to the fact that the geocentric perigee distance $r_{\mathrm{p}}$ remains (approximately) constant

$$
r_{\mathrm{p}}=a(1-e)=\text { const. }
$$

The eccentricity and the mean motion $n$ are therefore correlated, too. With Kepler's third law

$$
n^{2} a^{3}=\mu,
$$

where $\mu$ is the product of the gravitational constant and the mass of the Earth, we get

$$
e=1-\frac{r_{\mathrm{p}}}{\mu^{1 / 3}} n^{2 / 3}
$$

In a similar way we get an expression for the eccentricity of orbits which have their apogee at a fixed geocentric distance $r_{\mathrm{a}}$, e.g., at the GEO altitude

$$
\begin{aligned}
r_{\mathrm{a}} & =a(1+e)=\mathrm{const}, \\
e & =\frac{r_{\mathrm{a}}}{\mu^{1 / 3}} n^{2 / 3}-1 .
\end{aligned}
$$




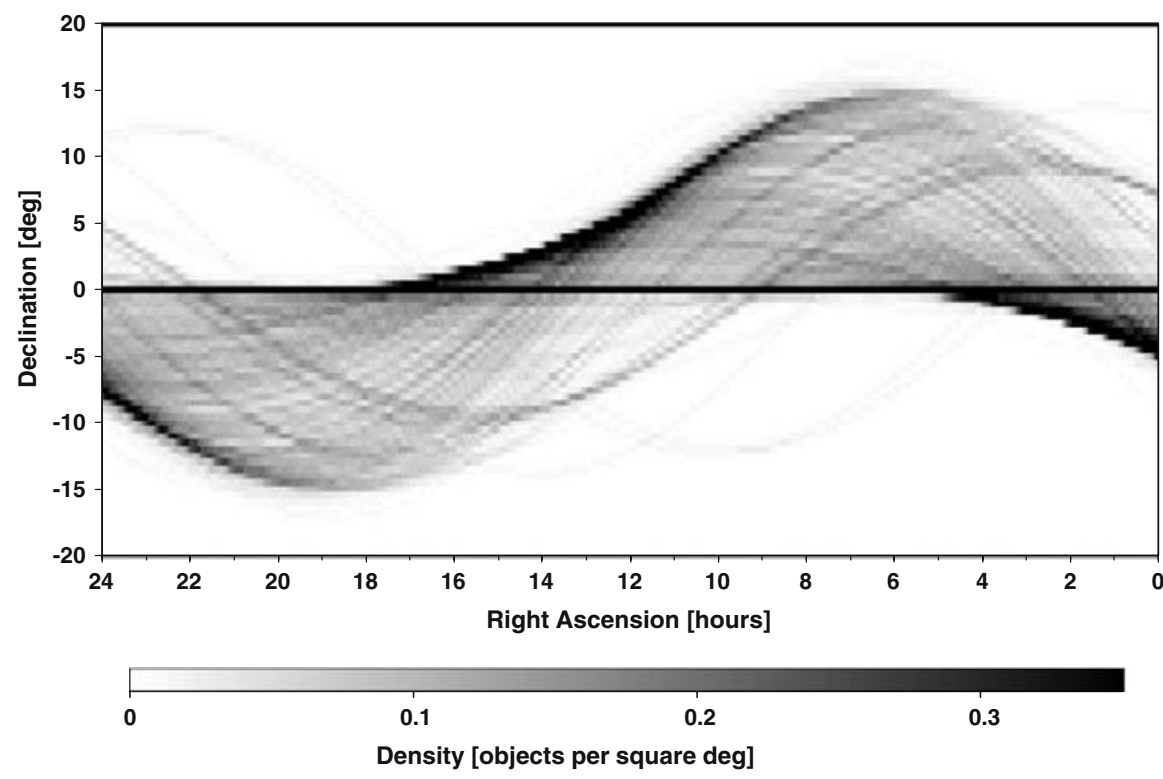

Fig. 23 Apparent density of the cataloged GEO objects in the right ascension-declination-space as seen from the geocenter. The density is given in objects/deg ${ }^{2}$ (status 2001)

\subsubsection{Apparent mean density of cataloged objects at the sky}

The search capacity of the current space debris surveys is rather limited due to the small field of view of a few square degrees of the used telescopes. It is therefore particularly important to know the apparent region where the objects of interest reside in order to best utilize the survey sensors.

It is reasonable to adopt the hypothesis that the cataloged GEO and GTO objects trace the debris population. The detailed orbital characteristics of these two populations, the 'cataloged objects' and the 'debris population', will almost certainly differ in important aspects (we expect, e.g., debris from distinct explosion events). But in general we expect the two populations to occupy roughly the same region in the orbital element space. Objects in other highly elliptical orbits, as well as objects in current navigation satellite constellation regions (MEO) occupy a much larger region.

Figure 23 illustrates the apparent density of the cataloged GEO objects in the right ascension-declination-space as seen from the geocenter. The catalog data was extracted from ESA's Database and Information System for the Characterization of Objects in Space (DISCOS), which contains orbital element data collected by the USSTRATCOM. The sinusoidal structure is produced by the mentioned precession of the orbital planes. The diagram gives the mean densities averaged over about $24 \mathrm{~h}$. Individual objects would move through the diagram from right to left within about $24 \mathrm{~h}$ along a sinusoidal path corresponding to their orbital inclination. The maximum density is found at the equator 


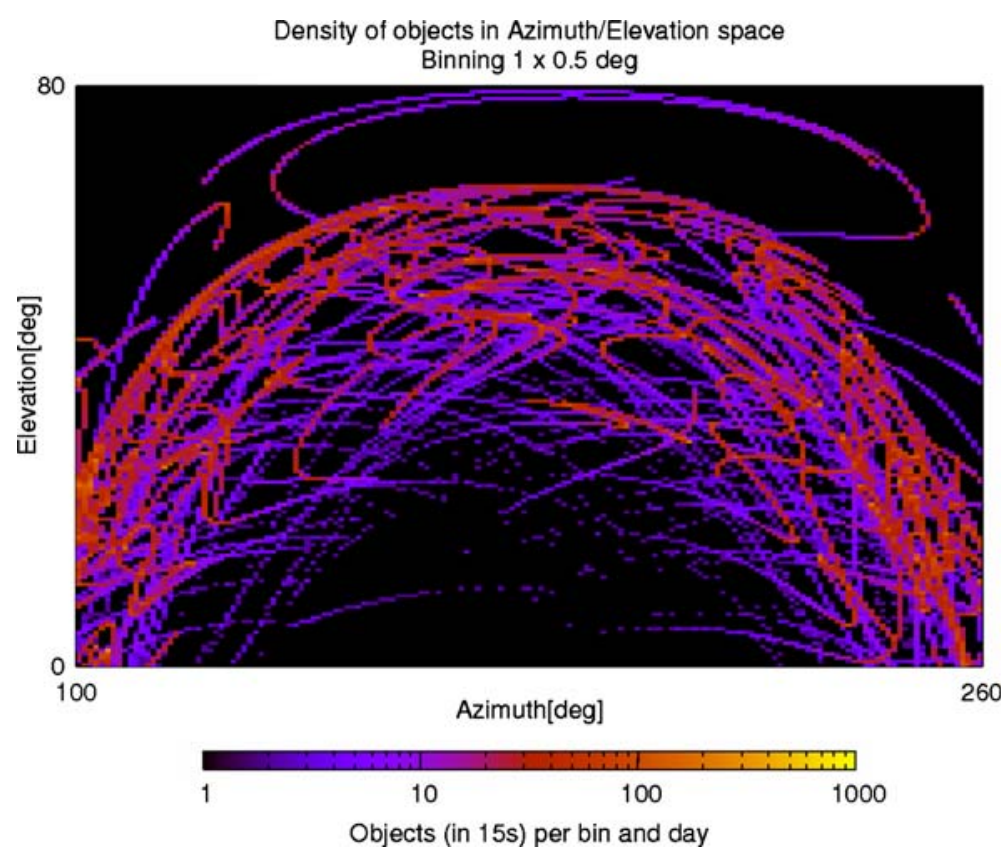

Fig. 24 Apparent density of cataloged GTO objects for the ESA observing site in Tenerife on the night of 21 July 2002 . Bin size is $1^{\circ}$ in azimuth and $0.5^{\circ}$ in elevation

where most active spacecrafts are located. The inclination of these objects is actively controlled, i.e., maneuvers are used to prevent the precession of the orbital plane.

In order to assess the apparent density of GTO objects on the sky in the horizon system, a simulation was performed for the ESA observing site in Tenerife. The simulation uses the DISCOS orbital element set of 3 May 2002, filtered for GTO objects with

eccentricity $e=\{0.5,0.9\}$,

inclination $i=\left\{0^{\circ}, 20^{\circ}\right\}$,

mean motion in revolutions per day $n=\{0.3,3.0\}$.

The filtered data set was then used as input to generate pass information and ephemeris data for the night of 21 July 2002. A total of 189 objects turned out to be visible during the night of 21 July. Figure 24 gives the apparent density of the objects for this night.

Due to the small bin size in these figures, passes of individual objects may be recognized. Figure 25 shows the same data, but with a bin size of $2^{\circ}$ in azimuth and elevation.

\subsection{Sky surveys for moving objects}

A so-called 'sky survey' is monitoring the sky for temporal changes, i.e., for objects, which have changed their brightness and/or their position. In the case 


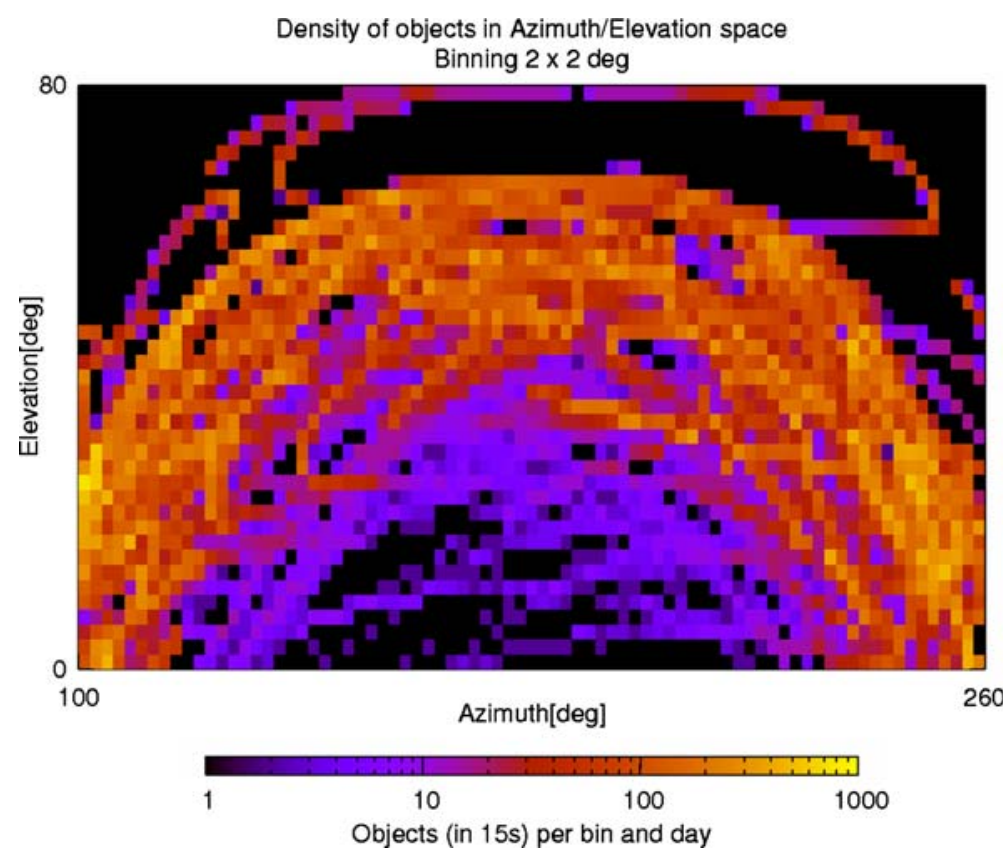

Fig. 25 Apparent density of cataloged GTO objects for the ESA observing site in Tenerife on the night of 21 July 2002. Bin size is $2^{\circ}$ in azimuth and $2^{\circ}$ in elevation

of a survey for moving objects we are looking for objects, which move with respect to the stellar background. Sky surveys are performed to search for a multitude of celestial objects including variable stars, supernovae and novae, as well as planets, minor planets and comets in the solar system. The angular velocities of moving objects range from a few arc seconds per day for Transneptunian objects, over a few tenths of a degree per day for main belt minor planets to several degrees per hour for near-earth objects (NEOs) during a close approach with the Earth. Space debris at high altitudes, e.g., debris in GEO, exhibit apparent velocities of $5-10^{\circ} / \mathrm{h}$ and are thus from the observational point of view comparable to the most extreme NEOs.

Moving objects may be detected either by searching for elongated images on a single, sidereally tracked frame or by searching for objects which changed their position on frames taken at different epochs. Most surveys for solar system objects use the latter approach. A few surveys use it in combination with streak detection algorithms to identify NEOs during close approaches. A majority of the extremely fast NEOs are, however, still confirmed by manually inspecting the frames. The classical approach used by all major NEO survey projects consists in taking three to five sidereally tracked exposures of the same star field. The exposure times are of the order of several $10 \mathrm{~s}$ and the exposures are spaced by several ten minutes to allow for a sufficient displacement of main belt objects from one exposure to the next (see, e.g., Stokes and Evans 2002). 
Surveys for space debris use a similar approach with slightly different time scales. The integration times are of the order of a few seconds and the gaps between the exposures are reduced to a few 10 seconds in order to catch the fast moving objects on more than one frame.

\subsection{Surveys for high-altitude space debris}

In the following sections we describe the technique used by the ESA survey for debris in GEO and GTO. The techniques used by other space debris surveys are similar. Essential differences are mentioned in the text. The following sections will address some important technical issues of the surveys.

A typical survey series is processed in three steps:

1. Search for moving objects.

2. Determination of celestial coordinates for all moving objects (astrometry).

3. Orbit determination and correlation of found objects with catalog; display of results.

If an object with a reasonable apparent motion is found on a series of frames (step 1) its position in a celestial reference frame is determined on each exposure by means of reference stars (step 2). By 'reasonable' we mean 'within the range of expected apparent motion for a near GEO object'. Issues concerning the scanning and object detection techniques are discussed in the Sects. 3.3.1, 3.3.2, 3.3.3, 3.3.4, 3.3.5 and 3.3.6, observational constraints are mentioned in Sect. 3.3.7, the astrometric calibration is addressed in Sect. 3.3.8.

Using the astrometric positions of the moving objects an initial orbit is determined and the orbital elements and the positions are compared with a catalog of known GEO objects, e.g., with DISCOS (step 3). (The comparison with a catalog is often also called 'correlation' with a catalog.)

The orbit determination and the catalog correlation process are discussed in Sect. 3.3.9.

Note that at the ESA telescope the entire processing is performed in near real time, which allows to follow-up new objects within 15-30 min after their discovery. These follow-up observations are essential for objects with a considerable eccentricity because the eccentricity cannot be determined from the discovery observation alone. From the technical point of view this real-time processing is quite demanding. A single full frame from the ESA telescope contains 32 Megabytes of data and the system easily acquires 1,000 frames during one night, which results in 32 Gigabytes per night! Considering the amount of frames it is obvious that the entire processing had to be automated to a great extent.

Figure 26 shows an enlargement of a part of a typical search frame from the ESA survey including a 12th magnitude GEO object. 


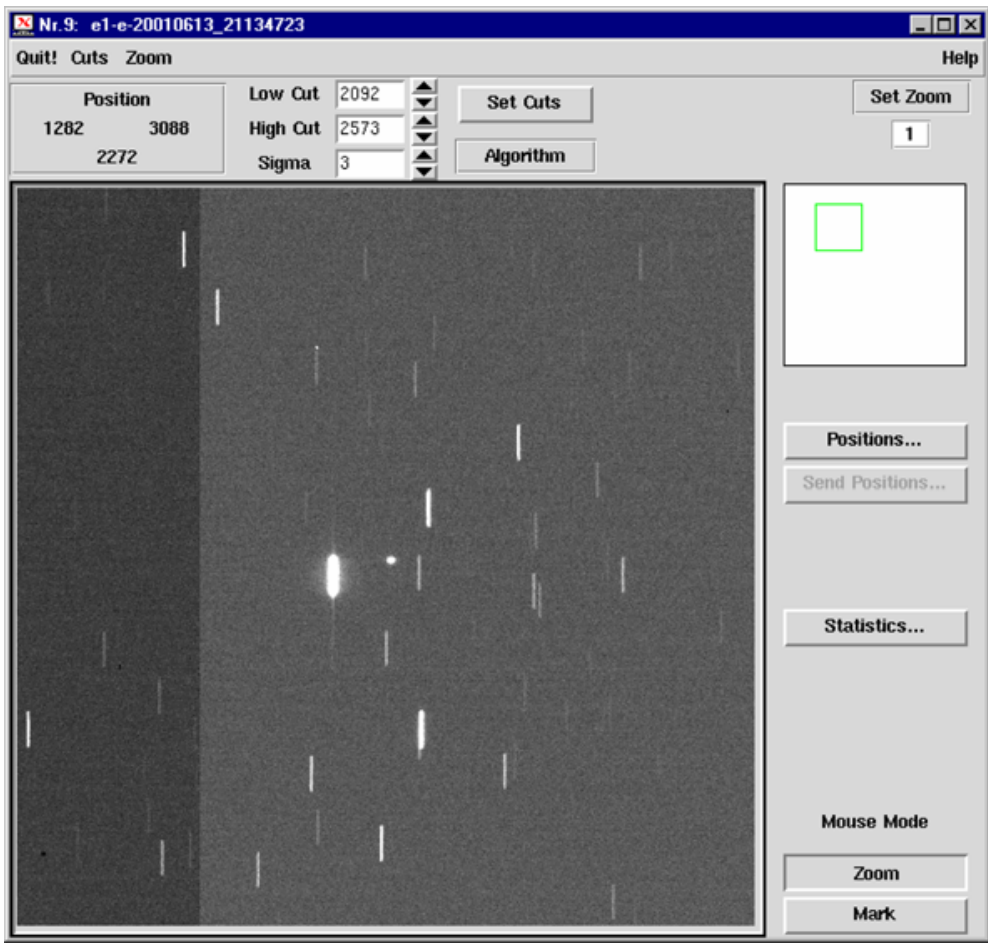

Fig. 26 Enlargement of a part of a search frame showing a 12th magnitude GEO object (point-like image at center). Stars produced trailed images in this 2-s exposure. The Figure shows about 1/16 of the entire field of view

\subsubsection{Scanning of declination stripes}

The detection technique is based on an algorithm comparing several consecutive frames of the same field in the sky. Fixed background stars are identified on a series of frames (10-30 frames) and the remaining part of the frames scanned for any additional objects. In order to optimize the signal-to-noise-ratio (SNR) for the objects of interest they are tracked during the exposures, which, in the case of GEO objects, means that the telescope is stopped. For GTO objects the tracking is of the order of 5-10 arcsec/s in hour angle (Schildknecht et al. 2004). As a consequence the stellar background is drifting across the field of view during the exposure, or in other words the stars leave trailed images on the frames. After each exposure the telescope is moved in a way that the same area of the sky is passing the field of view at the next exposure (see Fig. 27). With this method the telescope slowly scans the GEO ring from east to west at a fixed declination, while it is following the stars.

The optimization of the signal-to-noise ratio for faint GEO objects is a critical issue. If the object's light is integrated onto the same pixels and if the readout noise of the CCD detector is negligible, the SNR increases with the square root 


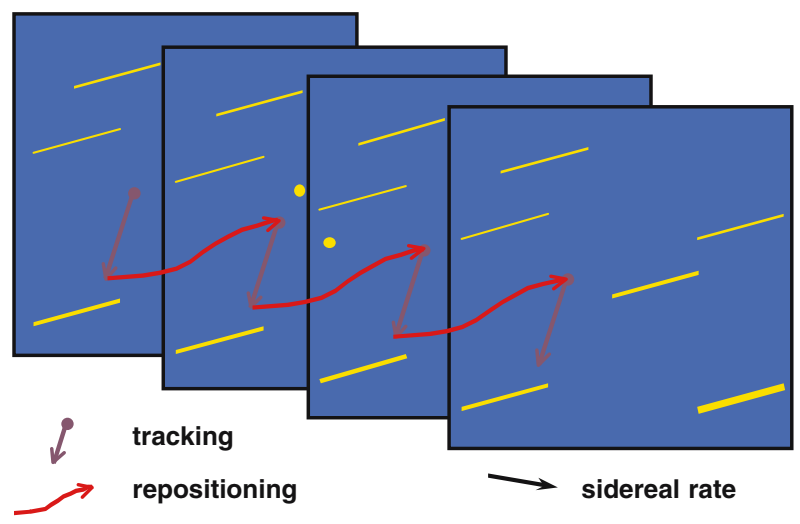

Fig. 27 Tracking scenario for surveys. The telescope is tracking the object with its expected motion during the exposure and is repositioned between the exposures in order to always observe the same field in the sky

of the exposure time. If, on the other hand, the object is moving with respect to the pixels, the maximum achievable SNR is limited by the background signal noise.

The CNES TAROT and the BSGC surveys use sidereostatic tracking which does not require a repositioning of the telescope and thus simplifies the telescope operations, but reduces the limiting magnitude for the moving objects. The MODEST survey uses a drift scan method to track the objects on the CCD detector while the telescope is constantly tracking the stars.

\subsubsection{Masking technique}

The so-called 'masking technique' uses a template frame (also called 'mask') to mask all background stars on the search frames. The unmasked parts of the masked search frames are then scanned for objects. The process is illustrated in Fig. 28.

A template or mask may either be generated from a single 'reference' frame or from a series of frames of the same field in the sky. In the first case the frame may be acquired with a longer exposure time than the search frames, in the second case the series of frames may be median averaged thereby generating a 'median averaged' reference frame. Both procedures lead to a reference frame with an increased SNR for the objects to be masked (stars). The mask can even be generated from a median frame of the search series because the median frame will only contain objects that did not move during the entire series.

Usually the individual parts of the mask corresponding to the individual stars are intentionally made slightly larger than actually determined from the reference (median) frame. This improves the probability that the stars on the search frames are still 'safely' masked even when their intensities or positions slightly changed with respect to the reference frame due to variations of the atmospheric transparency or due to jitter in the telescope tracking. 'Enlarging' the mask reduces the usable fraction of the search frames. This is, however, not 


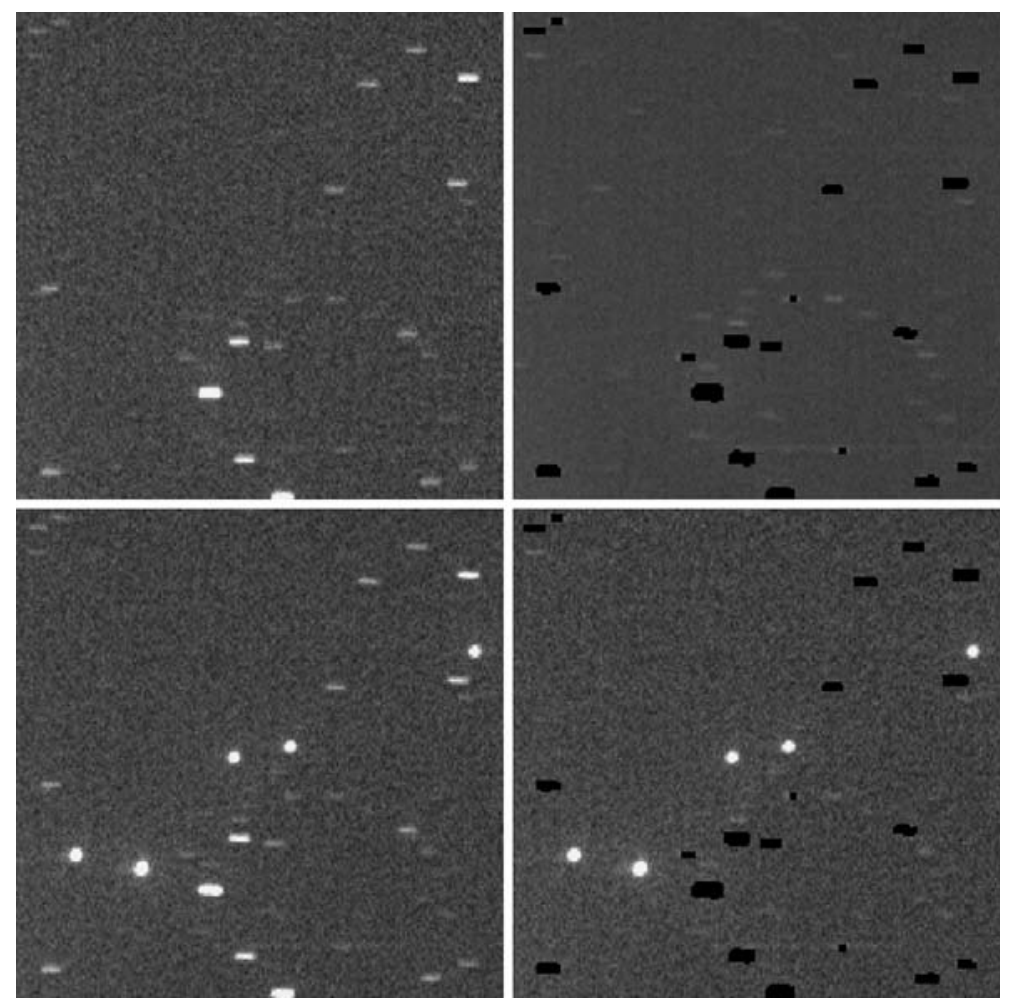

Fig. 28 Masking technique illustrated by two frames taken at the Zimmerwald 1-m ZIMLAT telescope. A reference frame (top left) is used to generate a mask (top right), which is applied to a search frame (bottom left). Five geostationary satellites (Astra A-E) are visible on the masked search frame (bottom right). The estimated magnitudes of the objects range between $12 \mathrm{mag}$ and $13 \mathrm{mag}$. The frames cover $7^{\prime} \times 7^{\prime}$ and were exposed for $1 \mathrm{~s}$

critical as the masks are usually covering less than $10 \%$ of the search frames. The reduced sensitivity to slight changes from exposure to exposure constitutes the essential advantage of the masking technique compared to the traditional 'subtraction technique' where a reference frame is subtracted from the search frames. The masking technique may, of course, be combined with the subtraction technique.

The process to generate the mask and the process to search for moving objects on the masked search frames use the same algorithms to detect objects. These algorithms use spatial filters and a local background determination to detect objects with signal-to-noise-ratios as low as two. The algorithms are also optimized to keep the 'false alarm' rate at an acceptable level. Additional filters are used to reject the so-called cosmic ray events, which are discriminated by virtue of the shape of the intensity profile.

The masking technique and the corresponding object detection algorithms which consists the heart of the observation technique - have been both developed at the AIUB (Schildknecht et al. 1995a). 
Most of the NEO surveys as well as most space debris surveys use a different technique, which is based on the extraction of objects from single frames. The result of these object extraction procedures is a list of objects with their coordinates on the frame and additional characteristics like the magnitude and the object image shape for each exposure. These lists are cross-correlated in a separate step to identify moving objects. The cross-correlation is usually performed for three consecutive frames of the same star field. Many processing systems are built around the open source (GPL) software 'SExtractor' developed by E. Bertin of the Institut d'Astrophysique de Paris (Bertin, Sextractor User manual, http://terapix.iap.fr/IMG/pdf/sextractor.pdf). The objects extraction approach has certain advantages compared with the masking technique. The latter, however, is performing better in crowded fields and in case of trailed star or object images.

Some NEO surveys use the mentioned subtraction technique where a median of all frames of the series is subtracted from the individual search frames. This method has some advantages in discriminating fast moving objects but the increased SNR of the median increases the overall SNR of the objects of interest.

\subsubsection{Exposure time}

The optimum exposure time for moving objects is about three pixelcrossing times (assuming that the point spread function is of the order of 2 pixels) (Schildknecht et al. 1995b). When the telescope is fixed during the exposure the pixel-crossing time theoretically would be indefinitely long for an ideal GEO object. A long exposure time, on the other hand, produces long star trails and thereby reduces the usable fraction of the frames considerably (the parts covered by background star trails will be masked). For the ESA telescope an exposure time in the range between 1 and $5 \mathrm{~s}$ is used. With the telescope fixed this leads to star trails with a length of 13-63 (binned) pixels. (During survey operations regions of $2 \times 2$ original CCD pixels are binned on the CCD during readout of the detector to produce pixels with a size of 1.2 arcsec.) The star trails during GTO surveys will be shorter by up to a factor of 2. Trails of geostationary satellites (angular velocities up to $4.5^{\prime \prime} / \mathrm{s}$ in the Earth-fixed frame) will have maximum lengths of 4-18 pixels (see Table 3 ). The lengths of the trails from GTO objects will have a larger range.

The optimum exposure time emerges from a trade-off between the length of the star trails (i.e., the reduction of the unmasked part of the frame) and the signal-to-noise ratio of faint objects (i.e., the detection limit for orbiting objects). It must be found as a function of the star density in the selected field.

\subsubsection{Overlapping}

For first orbit determination and for a safe distinction of moving objects from cosmic ray events an object should be observed two to three times (overlapping factor). Because the object may occasionally fall into the masked area of a frame 
Table 3 Length of star trails, maximum length of trails of geostationary objects, and apparent motion of an object leading to a trail length of 3,2 $\times 2$-binned pixels for different exposure times and fixed telescope

\begin{tabular}{llll}
\hline & \multicolumn{3}{l}{ Exposure time (s) } \\
\cline { 2 - 4 } & 1 & 3 & 5 \\
\hline Length of star trails (motion: $\left.15^{\prime \prime} / \mathrm{s}\right)($ binned pixels) & 13 & 38 & 63 \\
Max. length of GEO object trails (4.5"/s) (binned pixels) & 3.6 & 11 & 18 \\
Motion of object producing a trail of three binned pixels (arcs/sec) & 3.6 & 1.2 & 0.7 \\
\hline
\end{tabular}

a threefold coverage is desirable. An Earth-fixed survey volume should thus be observed two to three times. A given geostationary object will then be observed two to three times. The corresponding factor for GTO objects observed at apogee is up to two times larger (GTO objects at apogee move slower than GEO objects).

Given by the motion of the sky a stellar field takes $2.8 \mathrm{~min}$ to move through the field of view of $0.7^{\circ} \times 0.7^{\circ}$ of the ESA telescope when the telescope is fixed. If the telescope is tracking sidereally it takes the same amount of time for a geostationary object to move through the field of view. For a two- and a threefold coverage of the field, an exposure is required every 1.4 min or every $56 \mathrm{~s}$, respectively.

\subsubsection{Multi-stripe scanning}

If the combined exposure and detector readout time is less than the time interval between two successive exposures of the same field as required by the desired overlapping factor, two or more declination stripes may be observed simultaneously (see Fig. 29). After exposing the first field the telescope is moving to the second field and then potentially to a next field until the first field has moved by the amount defined by the overlapping factor. At this point the procedure is repeated. Using this multi-stripe scenario the area surveyed per unit of time is a multiple of the area surveyed if only one stripe is observed at a time. A separate mask has to be generated for each stripe.

Table 4 gives the available time per exposure for two-fold and three-fold coverage and different numbers of simultaneously scanned declination stripes. The current setup at the ESA telescope requires at minimum $30 \mathrm{~s}$ per exposure. In the normal survey mode we therefore scan two declination stripes simultaneously with nearly three fold coverage.

This scheme is easily adapted for NEO surveys and in fact turns out to be essentially identical to the classical minor planet search schemes. If we require a three fold coverage and furthermore assume an exposure time including the readout time of $2 \mathrm{~min}$ and a maximum motion of the objects of $0.3^{\circ} / \mathrm{h}$ we may observe 22 stripes in parallel. Each of these 22 stripes, i.e., celestial fields, would then be re-observed every $45 \mathrm{~min}$. Such an adapted survey scheme for minor planets and NEOs has been successfully applied (Schildknecht et al. 2001a). 


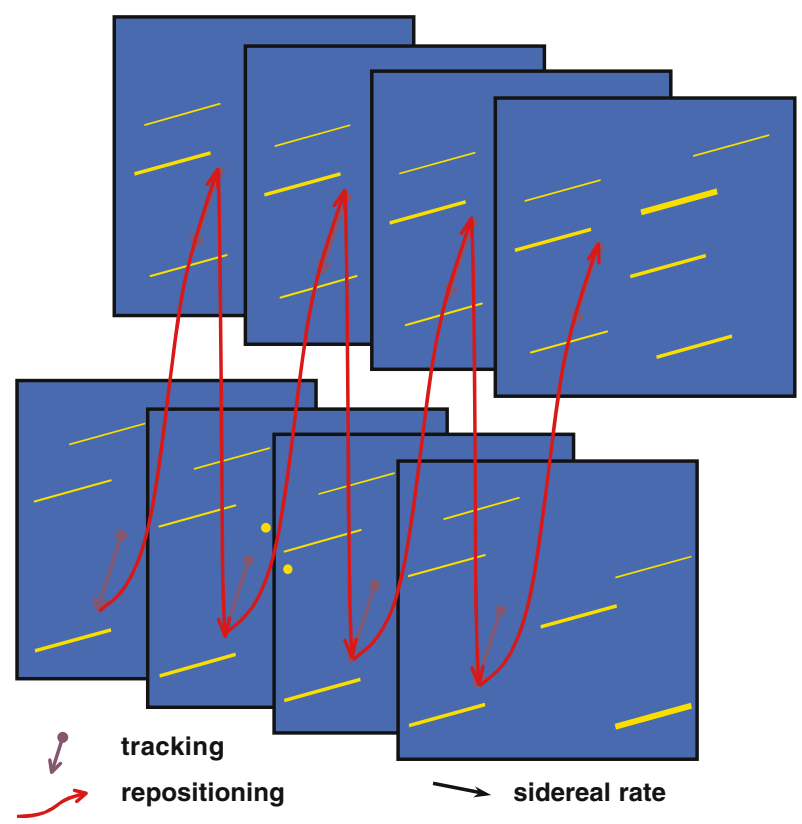

Fig. 29 Observation sequence for the simultaneous scanning of two declination stripes

Table 4 Time available per exposure for two fold and three fold coverage of one to six simultaneously scanned declination stripes (GEO objects, field of view of $0.7^{\circ} \times 0.7^{\circ}$ )

\begin{tabular}{lllllll}
\hline Number of declination stripes & 1 & 2 & 3 & 4 & 5 & 6 \\
\hline Two fold coverage (s) & 84 & 42 & 28 & 21 & 17 & 14 \\
Three fold coverage (s) & 56 & 28 & 19 & 14 & 11 & \\
\hline
\end{tabular}

\subsubsection{Surveyed area and data rate per hour}

The number of frames, the surveyed area and the data rate per hour for different setups of the ESA surveys is given in Table 5. Note that the data rate is given for $2 \times 2$-binned frames from the $4 \mathrm{k} \times 4 \mathrm{k} C \mathrm{CD}$ mosaic and would be four times higher for full frames.

\subsubsection{Observational constraints}

A number of observational constraints have to be taken into account when performing sky surveys for high-altitude objects.

Elevation of the survey field: Observations should be performed at high elevations, if possible, in order to minimize atmospheric extinction. For a clear atmosphere the extinction is about 0.20 mag per unit air mass (zenith) for visual magnitudes (Allen 1973) corresponding to a reduction factor of the intensity of 1.20 and a corresponding increase of the minimum detectable object size. 
Table 5 Number of frames, surveyed area and data rate per hour for $2 \times 2$-binned frames from the $4 \mathrm{k} \times 4 \mathrm{k}$ CCD mosaic for different overlapping factors and different number of declination stripes scanned simultaneously (ESA surveys)

\begin{tabular}{lllll}
\hline Number of stripes & Coverage & Frames/h & Area/h $\left(\mathrm{deg}^{2}\right)$ & Data rate $(\mathrm{GB} / \mathrm{h})$ \\
\hline One stripe & Two fold & 43 & 10.5 & 0.36 \\
& Three fold & 64 & 10.5 & 0.54 \\
Two stripes & Two fold & 86 & 21.0 & 0.72 \\
& Three fold & 129 & 21.0 & 1.08 \\
Three stripes & Two fold & 129 & 31.5 & 1.08 \\
& Three fold & 189 & 31.5 & 1.62 \\
Four stripes & Two fold & 171 & 42.0 & 1.44 \\
\hline
\end{tabular}

Shadow from the Earth and the Moon: GEO/GTO objects should be observed close to the Earth's shadow in order to minimize their phase angle (angle observer-object-Sun) and therefore to maximize their apparent brightness. Care has to be taken, however, not to observe inside the Earth's shadow. At geostationary distance the Earth's shadow has a radius of $6,200 \mathrm{~km}$ (umbra) and $6,560 \mathrm{~km}$ (penumbra) corresponding to an angular radius of $9.8^{\circ}$ and $10.3^{\circ}$, respectively, around the parallax corrected anti-sun direction.

A geostationary satellite close to the Earth's shadow cannot be eclipsed by the Moon's core shadow, even if the Moon is at perigee. If the satellite is far outside the opposition it may, however, enter into the Moon's core shadow. On the other hand, the Moon's penumbra at geostationary distance has an angular diameter of $4.9^{\circ}-5.5^{\circ}$. A geostationary satellite near opposition may therefore enter the Moon's penumbra only within $\pm 12 \mathrm{~h}$ around New Moon.

Objects in the geostationary ring may enter the Earth's shadow cone or the Moon's penumbra during more or less extended time periods around the solstices. The duration of these so-called eclipse seasons depends on the inclination of the object's orbit. For low inclination orbits the eclipse periods extend over about 2 months centered on 21 March and 23 September. For high inclination orbits the shadowing periods are correspondingly longer.

Milky Way: The masking technique requires rather sparse star fields otherwise the area that may be scanned for moving objects is reduced by an unacceptable amount. Therefore, the Milky Way must be avoided. Table 6 gives average numbers per square degree of stars brighter than a given visual magnitude for different galactic latitudes (from Allen 1973). At latitudes $\pm 20^{\circ}$ the star density is reduced by a factor of two for the brighter and a factor of three for the fainter stars, with respect to the densities at the galactic equator. At a galactic latitude of $\pm 30^{\circ}$ the reduction factor is between 3 and 5. Experience tells that the fraction of the search frames covered by the mask is below $10 \%$ for galactic latitudes higher than $\pm 20^{\circ}$.

Moonlight: For an optimum performance of the sky survey moonlight must be avoided. Moonlight increases the sky background signal and thus reduces the detection limit. In addition, light from the Moon close to the telescope pointing direction causes rapidly changing sky background gradients. This may cause 
Table 6 Numbers per square degree of stars brighter than a given visual magnitude for different galactic latitudes (from Allen 1973)

\begin{tabular}{llllllllll}
\hline Visual magnitude & \multicolumn{2}{l}{ Galactic Latitude } \\
\cline { 2 - 10 } & $\pm 0^{\circ}$ & $\pm 5^{\circ}$ & $\pm 10^{\circ}$ & $\pm 20^{\circ}$ & $\pm 30^{\circ}$ & $\pm 40^{\circ}$ & $\pm 50^{\circ}$ & $\pm 60^{\circ}$ & $\pm 90^{\circ}$ \\
\hline 5 & 0.083 & 0.069 & 0.059 & 0.044 & 0.032 & 0.028 & 0.025 & 0.023 & 0.020 \\
7 & 0.69 & 0.59 & 0.50 & 0.37 & 0.29 & 0.25 & 0.22 & 0.20 & 0.18 \\
9 & 6.0 & 4.9 & 4.1 & 3.0 & 2.4 & 2.0 & 1.8 & 1.6 & 1.4 \\
11 & 54 & 43 & 34 & 24 & 18 & 15 & 13 & 11 & 9 \\
13 & 400 & 310 & 230 & 160 & 120 & 87 & 69 & 59 & 49 \\
15 & 2,600 & 2,000 & 1,500 & 890 & 600 & 410 & 320 & 250 & 190 \\
17 & 13,000 & 12,000 & 7,900 & 4,000 & 2,300 & 1,500 & 1,100 & 870 & 600 \\
\hline
\end{tabular}

difficulties in the determination of the background intensity required by the object recognition algorithms.

Bright objects: Bright stars and planets in the field of view may cause strong so-called 'blooming' effects on the CCD array (charge overflow of pixels and entire columns) and should therefore be avoided.

Telescope and dome constraints: The ESA telescope is installed on a so-called English mount inside a classical slit-type dome. Although the telescope may reach any direction above $10^{\circ}$ elevation care has to be taken that the observation sequences do not require time-consuming maneuvers of the telescope or the dome slit.

\subsubsection{Astrometric calibration}

An important step in the reduction of the observations of any object is the determination of its position (e.g., $\alpha$ and $\beta$ ) in a celestial reference frame. This is a prerequisite for a subsequent orbit determination. The process is also called 'astrometric reduction' and the positions are referred to as 'astrometric places'. This reduction consists in essence of the determination of parameters of a transformation between the reference frame on the celestial sphere and the coordinate system in the focal plane. Apart from the projection of the sphere onto a plane this transformation contains all kinds of distortions generated by the telescope optics, as well as characteristics of the 'measurement system' of the detector. In the case of the ESA telescope the 'measurement system' is actually a mosaic of four separate CCD detectors. The current transformation model part for the CCD mosaic contains parameters for the mutual position and orientation of the CCDs and two scale factors for the pixel sizes for each CCD. The model for the telescope optics includes terms for a tilt of the focal plane with respect to the optical axis, for a decentering of the main optical elements and for radial distortions (e.g., produced by coma aberration). For details we refer to Schildknecht et al. (1995b).

Most of the mentioned transformation parameters have to be determined for each frame separately. The properties of the optical system, in particular, may 
change on short time scales due to changing temperatures or due to flexure of the telescope structure depending on the pointing of the telescope. Some parameters of the CCD mosaic, however, proved to be stable over months and are therefore calibrated separately using dedicated calibration observations (this approach improves the accuracy of the parameters compared to using the normal science frames only). Both, the calibration and the standard 'frame by frame' reduction, use the celestial coordinates of astrometric reference stars (also called 'catalog positions') together with the measured positions on the CCDs of the same stars to determine the transformation parameters in a least squares adjustment. Currently, reference stars from the Tycho 2 and/or the Hipparcos catalog are used for all astrometric reductions (Høg et al. 1995; Perryman et al. 1997).

Applying the mentioned calibrations the accuracy of a single position measurement of a moving object obtained in the ESA survey is of the order of 0.5 arcsec.

\subsubsection{Orbit determination and correlation with catalog}

The determination of an orbit for the newly detected objects is a critical and non-trivial task. To first order the task consists essentially in finding a particular solution of the one-body version of the equations of motion:

$$
\ddot{\boldsymbol{r}}=-\mu \frac{\boldsymbol{r}}{r^{3}},
$$

where $\mu$ is the product of the constant of gravitation and the mass of the central body, $\boldsymbol{r}$ the pericentric position vector of the object (and $r$ its length) and $\ddot{\boldsymbol{r}}$ its second time derivative. If the length of the orbit arc is short (a small fraction of the revolution period), the orbit model (3.3.1) may be sufficient to represent the observations within the arc with sufficient accuracy. If the arc length is growing, the equations of motion become more elaborate. Symbolically, we write them as follows:

$$
\ddot{\boldsymbol{r}}=-\mu \frac{\boldsymbol{r}}{r^{3}}+\delta \boldsymbol{f}\left(t ; \boldsymbol{r}, \dot{\boldsymbol{r}}, p_{1}, p_{2}, \ldots, p_{m}\right)
$$

where $f(\cdots)$ is the perturbing acceleration and $p_{i}, i=1,2, \ldots, m$ are additional unknown parameters, so-called dynamical parameters of the orbit; we may think of them as parameters characterizing radiation pressure or atmospheric drag. Usually, one may assume that the absolute value of $\delta \boldsymbol{f}(\cdots)$ is much smaller (typically by a factor of the order of $10^{3}$ ) than the absolute value $u / r^{2}$ of the main term (which justifies their neglect for arc lengths much shorter than one revolution period). If the object motion is governed by equations of motion of type (3.3.2) we still use the classical Keplerian orbital elements as the unknowns characterizing the initial values of the parameter estimation process, but we have to interpret them as osculating orbital elements referring to a particular epoch $t_{0}$ (here selected as the initial epoch of the arc). The osculating elements 
referring to the epoch $t_{0}$ are derived from the position- and velocity-vectors $\boldsymbol{r}_{0} \doteq \boldsymbol{r}\left(t_{0}\right)$ and $\dot{\boldsymbol{r}}_{0} \doteq \dot{\boldsymbol{r}}\left(t_{0}\right)$ using the formulae of the two-body problem. The orbit to be determined may then be written as the solution of an initial value problem (as long as the orbit model is assumed to be purely deterministic):

$$
\begin{aligned}
\ddot{\boldsymbol{r}} & =-\mu \frac{\boldsymbol{r}}{r^{3}}+\delta \boldsymbol{f}\left(\boldsymbol{t} ; \boldsymbol{r}, \dot{r}, p_{1}, p_{2}, \ldots, p_{m}\right) \\
\boldsymbol{r}\left(t_{0}\right) & =\boldsymbol{r}\left(a\left(t_{0}\right), e\left(t_{0}\right), \ldots, T_{0}\left(t_{0}\right)\right) \\
\dot{\boldsymbol{r}}\left(t_{0}\right) & =\dot{\boldsymbol{r}}\left(a\left(t_{0}\right), e\left(t_{0}\right), \ldots, T_{0}\left(t_{0}\right)\right) .
\end{aligned}
$$

When determining a first orbit we do not try to reduce the non-linear orbit determination problem to a linear one, but make instead the attempt to directly solve the non-linear problem. We use a modified Gauss method to solve a boundary value problem:

$$
\begin{aligned}
\ddot{\boldsymbol{r}} & =-\mu \frac{\boldsymbol{r}}{r^{3}}+\delta \boldsymbol{f}(t ; \boldsymbol{r}, \dot{\boldsymbol{r}}) \\
\boldsymbol{r}\left(t_{a}\right) & =\boldsymbol{o}\left(t_{a}\right)+\rho_{a} \boldsymbol{e}_{a} \\
\boldsymbol{r}\left(t_{b}\right) & =\boldsymbol{o}\left(t_{b}\right)+\rho_{b} \boldsymbol{e}_{b},
\end{aligned}
$$

where the boundary epochs $t_{a}$ and $t_{b}$ must be selected as two observation epochs (not necessarily the first and the last one). The indices $a$ and $b$ thus characterize two observation numbers. $\boldsymbol{o}(t)$ is the barycentric position vector of the observer at epoch $t, \boldsymbol{e}_{a}, \boldsymbol{e}_{b}$ are the observed unit vectors and $\rho_{a}, \rho_{b}$ are the (originally unknown) distances. The osculating elements as unknowns are replaced by the following six parameters (as auxiliary unknowns to be determined first):

$$
\left\{p_{1}, p_{2}, \ldots, p_{6}\right\}=\left\{\rho_{a}, \rho_{b}, \alpha_{a}, \alpha_{b}, \delta_{a}, \delta_{b}\right\},
$$

where $\alpha_{a}, \alpha_{b}$, and $\delta_{a}, \delta_{b}$ are the right ascension and the declination of the observed astrometric places

$$
\begin{aligned}
\alpha & =\arctan \frac{e_{y}}{e_{x}} \\
\delta & =\arctan e_{z} .
\end{aligned}
$$

The details of the particular method used to solve the problem (3.3.4) are given in Beutler (2004).

The series of discovery observations from space debris surveys, however, is usually very short and consists of a few position measurements spread over a time interval of a few minutes. Given the orbital periods of the objects, which are of the order of 1 day, it is obvious that these extremely short arcs prevent determining full six-parameter orbits. There are in principle two possibilities to 
eventually obtain a full orbit in these cases, (a) acquire follow-up observations in near real time or (b) to plan the surveys in a way that most objects of interest will appear incidentally on several observation series during the same or during consecutive nights.

In the first case we may determine a circular orbit from the discovery observations and compute ephemerides to perform follow-up observations. Depending on the type of orbit (GEO, GTO, etc.) and the field of view of the sensor used for the follow-up these observations must be acquired within a time interval of $15 \mathrm{~min}$ up to several hours after the discovery (for details see Musci et al. 2005). Additional observations will then be required during the following night to finally derive an orbit allowing recovering the object after many revolutions. Using appropriate models for the gravitational and non-gravitational forces a GEO object may, e.g., be recovered after several months within a field of view of $1^{\circ}$ provided that the orbit has been determined from observations spanning an arc of several days. Similarly, an orbit determined from observations of a GTO object covering a time interval of several days allows recovering the object after a few weeks (in this case it is important that the observations cover a good part of the orbit in terms of anomaly).

In the second case all observations from different epochs, e.g., from different nights, have to be cross-correlated in order to find detections which belong to the same object. The latter is eventually best tested by trying to fit an orbit through all observations, which are supposed to stem from the same object. However, this process is computationally intensive and we need a method to pre-select candidate observations. Algorithms used to select these candidates should take into account the position and the apparent motion from the single detections and must confine the orbital element space to a region as small as possible but still appropriate for the objects of interest. Instead of the apparent motion we may also use the circular orbits determined from detection series, which - if properly treated - is equivalent.

The correlation with an existing catalog of orbits is finally done by comparing either the observed positions or the determined orbital elements, or a combination thereof, with the corresponding quantities derived from the catalog. This correlation should be based on the known accuracy of the catalog orbits, i.e., should make full use of possible covariance information.

The orbit determination and cataloging tasks for high-altitude space debris have many striking similarities with the corresponding tasks in surveys for minor planets. The orbital periods involved in space debris surveys are of the order of 1 day while classical minor planets and NEOs have revolution times of the order of 1 year. Using this scaling factor of roughly 500 a series of space debris discovery observations spanning 1 min would correspond to an arc of several hours for a minor planet. Similarly, a real-time follow-up observation after 15 minutes would correspond to a NEO followup after 5 days. Finally, in the case of high-altitude debris, the time interval from one night to the next is about the time interval between two oppositions. 


\section{The ESA survey for small-sized debris in high-altitude orbits}

\subsection{Introduction}

Recognizing the unique value of the geostationary region (GEO) for both commercial and scientific missions (Space Debris 1988; Flury 1988, 1991; Lobb et al. 1993; Interagency Report on Orbital Debris 1995) ESA established an optical search program to detect uncataloged space debris objects in GEO. The results of this survey program should first of all improve our understanding of the current space debris population in GEO by acquiring statistical information on the number of objects, their sizes and their orbital parameters. This information will enable analysts to identify the major sources of debris in this region and finally to devise the most effective approaches to mitigate debris in future. Eventually, a catalog of existing space debris in GEO shall be produced in order to increase the safety of active spacecrafts by allowing them to perform collision avoidance maneuvers if necessary.

Long-term monitoring of the GEO will furthermore allow to directly observe the evolution of the debris population in GEO, both in terms of number of objects and in terms of their orbits. This data in turn will be indispensable to validate space debris evolution models.

In the context of this program ESA has set-up a 1-m telescope at the Izaña observatory in Tenerife, Canary Islands (see Sect. 2.3.3, Fig. 14). In parallel, a study to develop algorithms for the optical detection of space debris was awarded to AIUB (Schildknecht et al. 1995b). AIUB also developed and implemented software systems for the observation planning, the so-called Level-1 telescope control and image data acquisition (Schildknecht et al. 2001b), as well as for the on- and off-line data processing (Schildknecht et al. 1998)

First observations took place in autumn 1999 and regular space debris surveys are performed since January 2001. The observations are acquired during 12-night-intervals centered on New Moon. During the last years about 80 to 100 observation nights per year were devoted to space debris surveys.

\subsection{Observation campaign characteristics}

All survey campaigns were performed using the 'declination stripe scanning' method described in Sect. 3.3.1. When defining the corresponding search fields a series of observational constraints had to be taken into account (see Sect. 3.3.7). Since 2002 part of the ESA surveys has been optimized to search for GTO objects (for details of the search scenarios see Schildknecht et al. 2004).

Figure 30 shows the survey fields of the spring 2001 campaign. The figure does not tell which (or how many) objects should be observable in a given field, but merely which orbital planes we may see in a particular field. An object in an orbital plane crossing a given survey field will cross this field once every day, but perhaps when the field is not visible from the ESA observing site in Tenerife (this is the case for GEO objects at longitudes not reachable from Tenerife). 


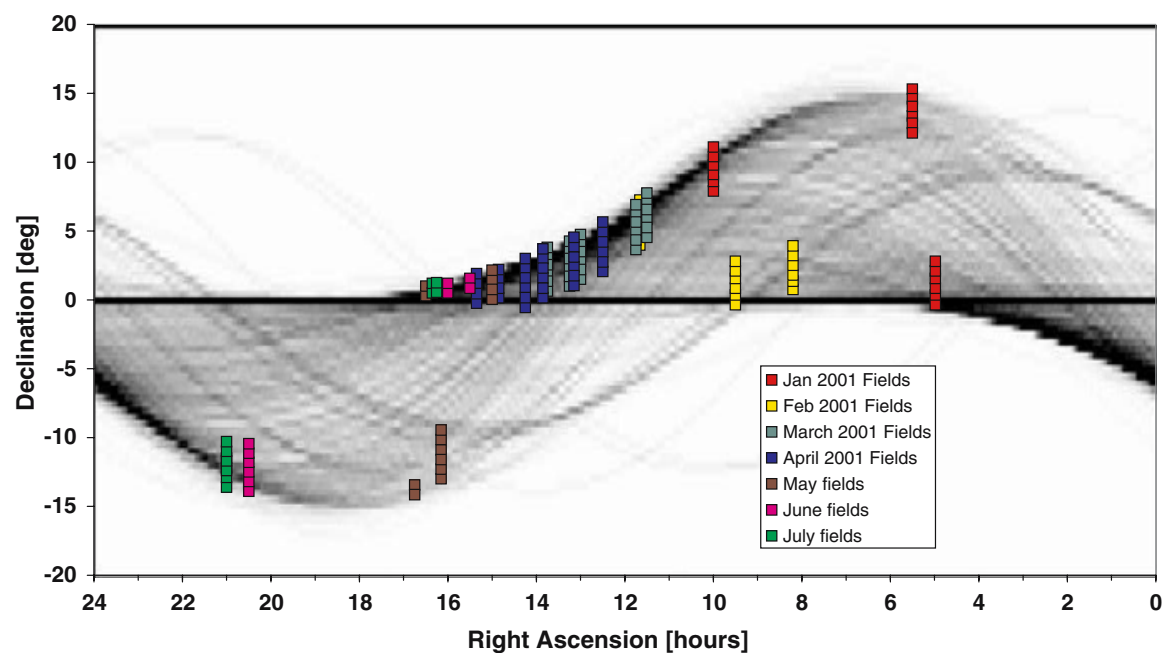

Fig. 30 Survey fields of the spring 2001 campaign (small squares) in the right ascension-declination space as seen from the geocenter. The gray-shaded background indicates the apparent density of the cataloged GEO objects

A limited set of follow-up observations of newly detected (uncataloged) objects was acquired during the surveys. The aim of these observations was primarily to allow for the determination of elliptical orbits for a small sample of the objects. (From the two or three detection observations we may safely determine a circular orbit only.) Follow-up observations were planned in real time by the observers and executed about $1-3 \mathrm{~h}$ after the detection of an unknown object.

\subsection{Results}

Table 7 gives an overview of the ESA GEO and GTO campaigns until December 2005. The table includes the 1999 test campaign, which consisted in fact of a first, very limited series of system tests (Flury et al. 2000). Nevertheless, the data from this campaign significantly contributed to the results of the first IADC GEO survey campaign (Africano and Schildknecht 2000). The table includes the data acquired for the second (January to March 2002, Africano and Schildknecht 2003), third (October 2002 to April 2003, Africano and Schildknecht 2004), and fourth (January to September 2004) IADC coordinated campaigns. The terms 'correlated' and 'uncorrelated' refer to objects/detections for which a corresponding catalog object could or could not be identified, respectively. The identification procedure, or 'correlation procedure', is based on comparing the observed and the catalog orbital elements and the position of the object at the observation epoch. We used the unclassified part of the USSTRATCOM catalog as our reference (actually data from the ESA DISCOS database was used). By 'detection' we denote the detection of an object 


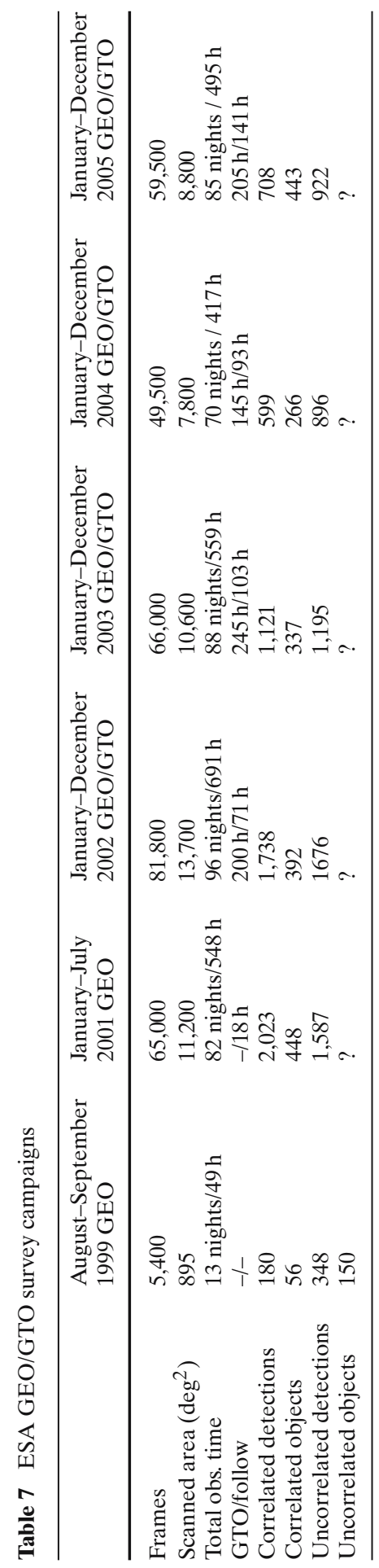


within a single 30-min observation series. Some of these detections may actually refer to the same object, i.e., we may have incidentally re-observed some of the objects previously. We subsume all detections that seem to belong to the same real object under the term 'correlated object' (if the object is part of the catalog) or 'uncorrelated object' (if the object is not part of the catalog), respectively, by mutually correlating all detections of a campaign. This latter task is identical with the creation and maintenance of a temporary catalog of orbital elements for the unknown objects and has been performed for the first campaign only (which explains the question marks in the last row of Table 7). The row labeled 'GTO/Follow' indicates the number of hours, which were dedicated to GTO surveys or used for follow up observations, respectively.

At this point it is very important to point out that all surveys in Table 7 suffer from observational biases. In other words 'what we see depends on where and when we look'. The numbers given in Table 7 could therefore be misleading, e.g., when simply taking the ratio of uncorrelated to correlated detections as a measure to estimate the total number of debris objects!

We present the essential results of this survey in the following sections by using a subset of the data covering the time interval from January 2004 to August 2005. Data from other time intervals would look similar. The debris environment is highly dynamic we therefore do not combine the data from the entire time interval, otherwise some signatures would become blurred. The full data set, on the other hand, allows monitoring temporal changes.

\subsubsection{Absolute magnitude distribution}

Figure 31 shows the histogram of absolute magnitudes of all detections from January 2004 to August 2005. The solid line indicates the system sensitivity (scale at right-hand side) as determined from independent calibration measurements. All magnitudes have been reduced from apparent magnitudes to so-called absolute magnitudes by correcting for the illumination phase angle. For the scattering properties we assumed a simple Lambertian sphere. No reduction to a common distance has been done because of the uncertainties of the determined orbits (see below). The value of this correction would be below 0.5 magnitudes in most cases. The magnitudes are astronomical ' $\mathrm{V}$ magnitudes' and have an accuracy of a few 0.1 magnitudes, except for the very faint objects where errors could amount to a maximum of 1 magnitude. The indicated object sizes were derived by assuming Lambertian spheres and albedo of 0.1 . Both assumptions, however, are uncertain, as long as we do not know the nature of the observed objects.

The distribution is bimodal. For the correlated objects the distribution has its peak at about magnitude 12.5 and spreads from about magnitude 10-15. It is also slightly asymmetric with the slope on the fainter end being shallower. This distribution nicely (and to some extent naturally) reflects the size distribution in the catalog. The uncorrelated objects seem to be concentrated in a broad strong peak around magnitude 18-18.5 and in a second much less 


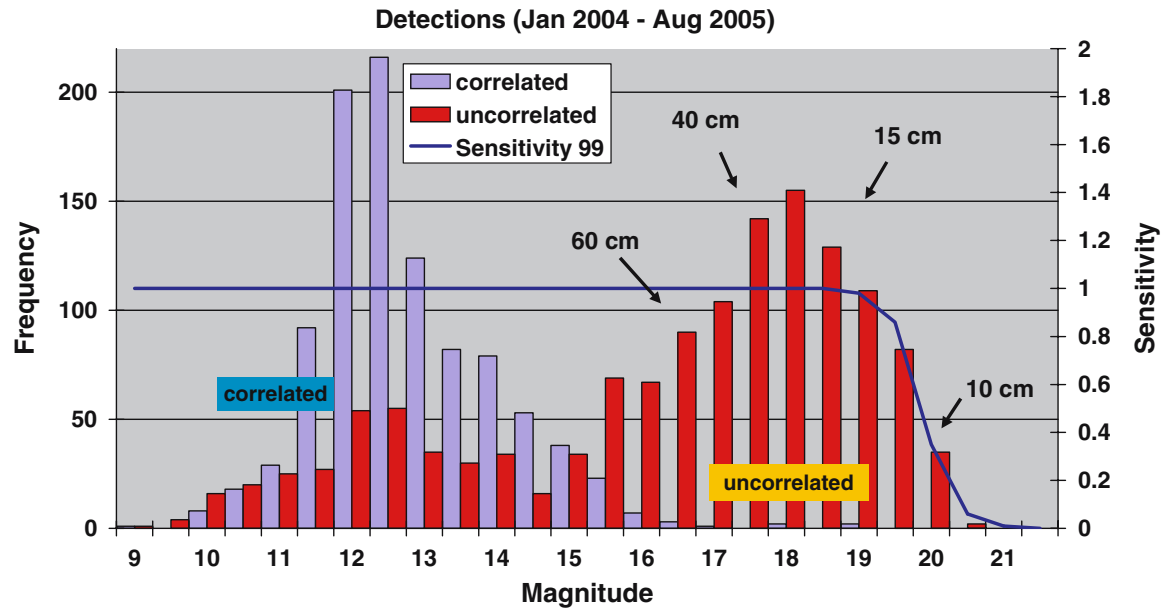

Fig. 31 Absolute magnitude distribution for the detections from January 2004 to August 2005. The solid line indicates the system sensitivity (scale at right-hand side) as determined from independent calibration measurements

pronounced peak, which follows more or less the distribution of correlated objects.

The bright objects in the latter peak are most likely all 'known', large objects, which did not correlate with the catalog for several reasons: some of these objects are classified and were therefore not included in the 'unclassified' version of the catalog. Others might well be in the catalog but did not correlate due to insufficient accuracy of the catalog or due to a recent maneuver of the object not yet reflected by the catalog at the time of observation. In many cases these objects are members of groups of satellites co-located in the same $0.1^{\circ}$ longitude slot. They often correlate with several objects in the catalog, again due to the limited accuracy of the catalog, and thus end up as 'uncorrelated' due to ambiguities. Also the catalog most likely is incomplete at its fainter end.

The uncorrelated objects in the range from magnitude 15-21 are smaller than the minimum size of the objects in the catalog. The apparent main peak of this population at about magnitude 18 is in fact not a peak, because the cutoff in the number of objects fainter than about magnitude 19 is entirely due to the sensitivity limit of the observation system (see the line indicating system sensitivity). The real luminosity function beyond magnitude 19 could therefore still increase!

The discovery of this substantial population of small-sized debris objects in high-altitude orbits is one of the major achievements of the ESA survey. The observed magnitude distribution for this time interval is consistent with the corresponding distribution of other time intervals of the survey (see, e.g., Fig. 15). Furthermore the data has been partly confirmed by other surveys (see Figs. 11, $12,13)$ but until now there is no independent data covering the magnitude region beyond magnitude 18 available. 
Inclination Distribution (Jan 2004 - Aug 2005)

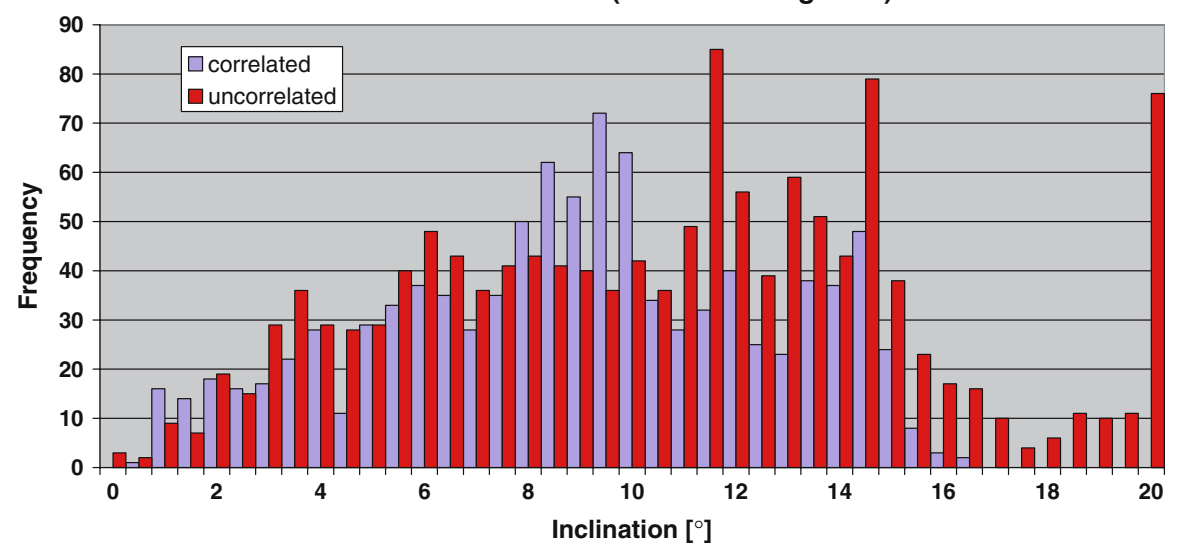

Fig. 32 Inclination distribution for the detections from January 2004 to August 2005

\subsubsection{Inclination distribution}

The inclination distribution for the detections from January 2004 to August 2005 is given in Fig. 32. The ratio of uncorrelated to correlated detections is roughly constant (about one) for inclinations smaller than about $8^{\circ}$. Between inclination eight and ten there is a surplus of correlated detections and for higher inclinations there is a clear excess of uncorrelated detections. There are even two distinct concentrations of uncorrelated objects around 12 and $15^{\circ}$ inclination. No correlated objects with inclinations larger than $17.5^{\circ}$ were found. The bin at $20^{\circ}$ includes all detections with inclinations larger than $19.5^{\circ}$. The survey included almost no search fields at low declinations, which resulted in very few detections with inclinations below $2^{\circ}$. In fact the catalog population has a strong peak at $0^{\circ}$ inclination where most active, station-keeping, i.e., controlled spacecrafts, are located (see Fig. 23).

\subsubsection{Distribution of semimajor axes}

Figure 33 shows the distributions of the so-called 'inferred' semimajor axes for the same data set. By the term 'inferred' we denote that these semimajor axes are in fact the radii of the circular orbits determined from the detection observations (the extremely short arc of the detection observations does not allow for a full six-parameter orbit determination). Both, the correlated and the uncorrelated objects, are concentrated around the nominal GEO semimajor axis. The semimajor axes of the uncorrelated objects, however, are much more dispersed showing a significant asymmetry with an excess at large values. The last bin at $50,500 \mathrm{~km}$ in fact contains all detections with semimajor axes larger than $50,000 \mathrm{~km}$.

It is important to keep in mind that inferred semimajor axes are in fact determined assuming circular orbits, which is certainly not the best guess for 
Semimajor Axes (Jan 2004 - Aug 2005)

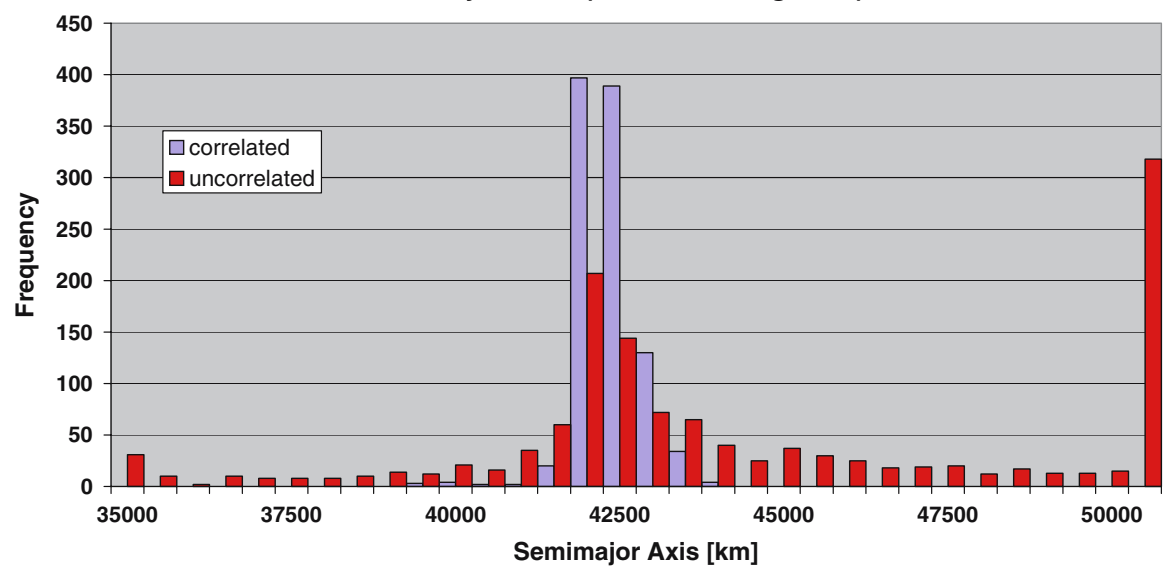

Fig. 33 Distribution of inferred semimajor axes for detections from January 2004 to August 2005

all objects. In general, fixing the eccentricity at a wrong value may result in a large bias of the inferred semimajor axis. Part of the spread for the uncorrelated objects, as well as some of the large values may be due to this effect. Objects on highly eccentric orbits having their apogee near GEO may mimic objects in much higher circular orbits when observed at apogee. This is in particular the case for objects on a geostationary transfer orbit (GTO). Fitting circular orbits through observations of GTOs near apogee yields inferred semimajor axes of the order of $50,000 \mathrm{~km}$ and larger, whereas the real values would be about $26,000 \mathrm{~km}$ but with an eccentricity of $e=0.73$ (see Sect. 4.4)!

\subsubsection{Inclination and right ascension of the ascending node}

We have seen in Sect. 3.1.1 that the inclinations $i$ and right ascensions of the ascending nodes $\Omega$ are strongly correlated for the catalog population. Figures 34 and 35 give both elements for all correlated and uncorrelated detections from January 2004 to August 2005. The distinct curve followed by the correlated objects is caused by the 53-year precession period of the orbital planes. Assuming that the objects started with orbits of $i=0^{\circ}$ inclination the actual position in the diagram stands for the time since the end of active inclination control. The orbits gradually evolve from low inclination and at right ascension of the ascending node of about $100^{\circ}$ to higher inclinations and smaller right ascension of the node until they reach the maximum inclination of $i=15^{\circ}$ after 26.5 years. The oldest catalog objects have already passed this point (see Fig. 21).

The correlated objects (Fig. 34) nicely show this evolutionary pattern. There are again no objects found at $i=0^{\circ}$ inclination because this region was not included in the survey. Uncontrolled objects lie predominantly on the line from $\Omega \approx 90^{\circ}, i \approx 0^{\circ}$ to $\Omega \approx 5^{\circ}, i \approx 15^{\circ}$. The latter point is the location where the objects reach the maximum value for the inclination about 26.5 years after 


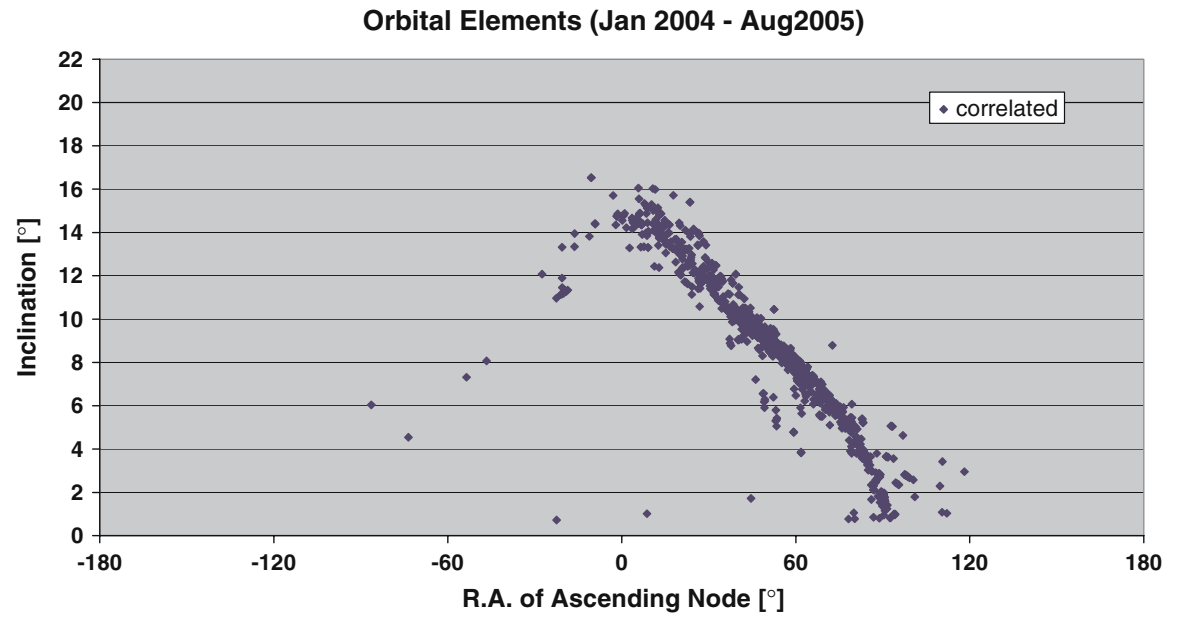

Fig. 34 Inclination versus right ascension of ascending node for the correlated detections from January 2004 to August 2005

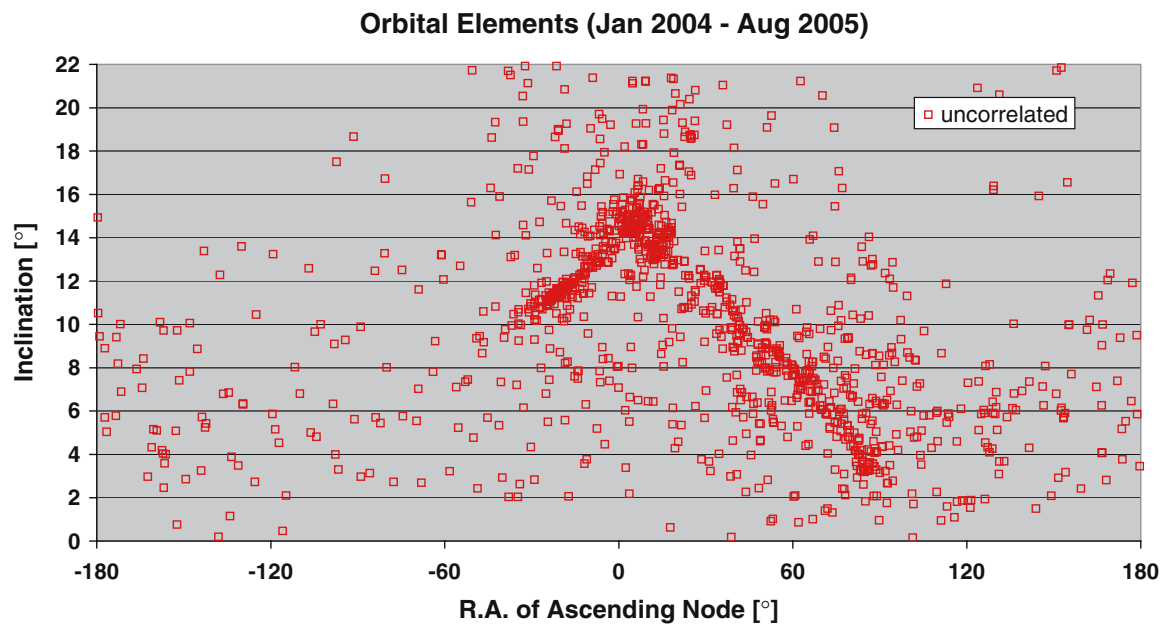

Fig. 35 Inclination versus right ascension of ascending node for the uncorrelated detections from January 2004 to August 2005

the end of active inclination control. A few objects already passed this point and are now in the $\Omega$-interval from $0^{\circ} \geq \Omega \geq-30^{\circ}$ at inclinations between $15^{\circ} \geq i \geq 10^{\circ}$.

The bulk of the uncorrelated objects (Fig. 35) lies on the mentioned evolution track but with a much larger spread. In addition, there is a 'background' component with a homogeneous distribution in the $(\Omega, i)$-space noticeable in Fig. 35. The most striking features, however, are the distinct clusters of objects. Prominent concentrations are found in Fig. 35 at $\Omega \approx 15^{\circ}, i \approx 13.5^{\circ}$, and $\Omega \approx$ $5^{\circ}, i \approx 14.5^{\circ}$ (both with an elliptical shape), and at $\Omega \approx-20^{\circ}, i \approx 11^{\circ}$ ('bananashaped'). Less prominent clusters are located at $\Omega \approx 85^{\circ}, i \approx 3.5^{\circ}, \Omega=63^{\circ}, i \approx$ 


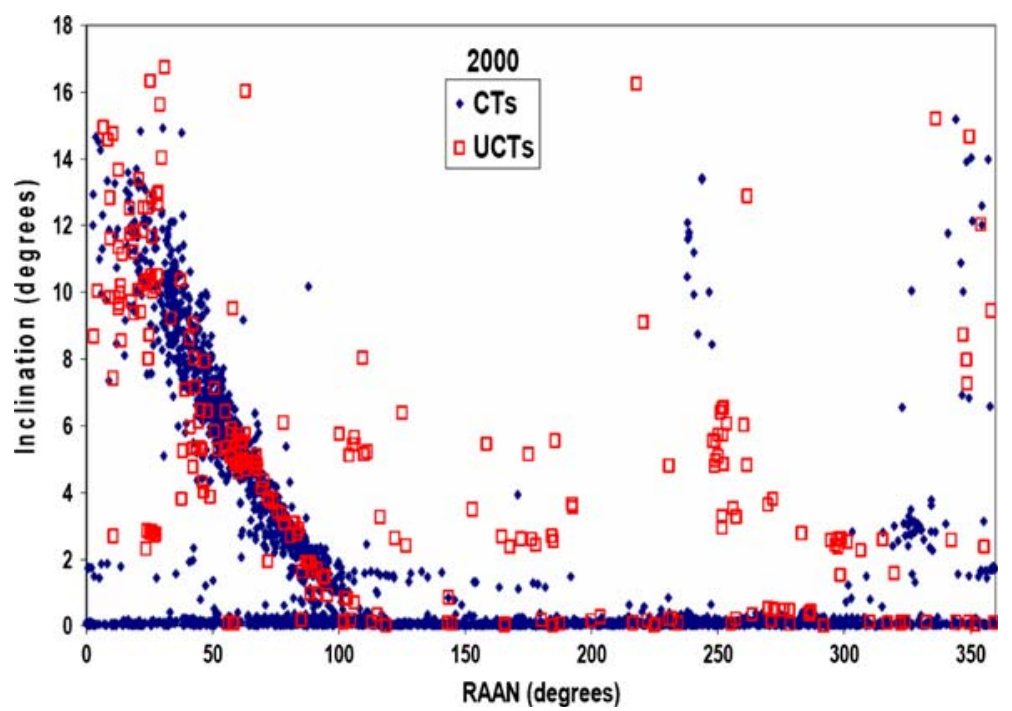

Fig. 36 Inclination versus right ascension of ascending node ('RAAN') for the CDT data of the year 2000 (from Barker et al. 2005). 'CTs' refers to the correlated and 'UTCs' to the uncorrelated detections

$7.5^{\circ}$, and $\Omega \approx 35^{\circ}, i \approx 12^{\circ}$. All mentioned clusters are also present in the data covering other time intervals.

We have checked some of the clusters for multiple sightings of one and the same objects but conclude that the clusters are real (a pure selection effect can thus be excluded). The only rational explanation for the origin of these clusters are explosive events. First attempts to reproduce these results by modeling a series of explosions have been performed (e.g., Wegener et al. 2001; Pardini and Anselmo 2005). However, still more data are needed to understand the details of the mechanism or to even trace the clusters back to their parent objects.

The explanation for the 'background' component is more difficult. A substantial part of this component may be an artifact due to the fact that both orbital elements are inferred from circular orbits. As explained in the paragraph on the distribution of the semimajor axes, objects on highly eccentric orbits like GTOs may affect the results and could at least partly be responsible for the 'background' component.

Figures 36 and 37 show $(\Omega, i)$-diagrams for the CDT and for the TAROT data (the MODEST survey does not provide orbital elements). Both surveys derived orbital elements assuming circular orbits. The correlated detections are predominantly following the evolution path in both surveys. The majority of the uncorrelated detections in the CDT data follows the distribution of the correlated detections. There are indications of clusters at $\Omega \approx 65^{\circ}, i \approx 5^{\circ}$, at $\Omega \approx 30^{\circ}, i \approx 12^{\circ}$, and at $\Omega \approx 25^{\circ}, i \approx 3^{\circ}$. None of these apparent clusters matches with a cluster seen in the ESA survey. However, the CDT detections were only checked so that they are 'unique within one night' but the clusters 


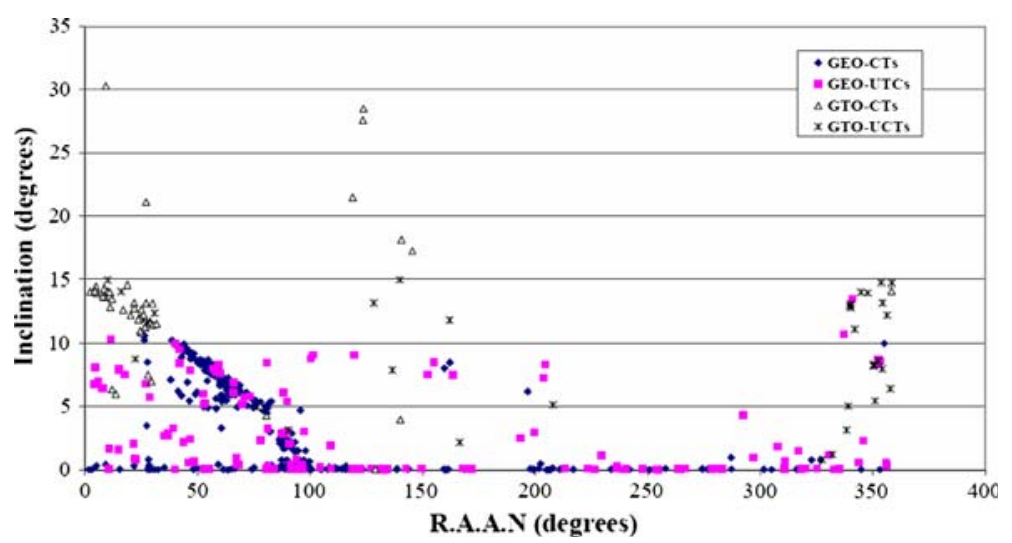

Fig. 37 Inclination versus right ascension of ascending node ('R.A.A.N') for the TAROT detections from the 2004 IADC GEO campaign (from Ríos Bergantiños et al. 2005). 'GEO-CTs' and 'GTO-CTs' refers to the correlated and 'GEO-UTCs' and 'GTO-UTCs' to the uncorrelated detections ('GEO' and 'GTO' do not refer to confirmed GEO or GTO objects respectively)

could be multiple detections of the same objects over the time span of the survey. In both surveys there is an indication of the rather evenly spread 'background' component of uncorrelated detections seen in the ESA survey. The clusters in the ESA data consist predominantly of faint objects beyond the detection limit of the CDT and the TAROT sensors, which explains that they are not seen by the latter. Any further comparison of the data sets requires a detailed analysis of the selection biases of the surveys.

\subsection{Surveys for objects in highly eccentric orbits}

One limitation of the results emerges from the fact that circular orbits had to be inferred. This is a good approximation for GEO objects. But for objects with highly eccentric orbits we have to expect that the true orbital elements differ considerably from the inferred circular orbits. The 'contamination' by elliptical orbits does in particular affect the distribution of semimajor axes and the orientation of the orbital planes, i.e., the inclination $i$ versus right ascension of the ascending node $\Omega((\Omega, i)$-distribution $)$.

In a GEO survey objects with high eccentricities are normally detected when they are near the apogee. By inferring circular orbits for these objects we in fact interpret the change in the true anomaly near the apogee as the mean motion of a circular orbit. The velocity of an object in an elliptical orbit at the apogee is slower than the corresponding velocity of an object on a circular orbit with a radius equal to the apogee radius of the former. This in turn means that the radius of the inferred circular orbit exceeds the apogee radius of the elliptical orbit.

More precisely what happens is that the rate of change in true anomaly $\dot{\theta}$ at apogee for an object in an elliptical orbit is interpreted as the mean motion $n$ of an object in a circular orbit. We use Kepler's second law to derive a relation 
between the change in true anomaly $\dot{\theta}$ and the geocentric radius $r$

$$
r^{2} \dot{\theta}=\text { const }=\sqrt{\mu a\left(1-e^{2}\right)},
$$

where $\mu$ is the product of the gravitational constant and the mass of the Earth, $a$ is the semimajor axis and $e$ is the eccentricity of the orbit. For an object at apogee we get $r=a(1+e)$ and thus

$$
\dot{\theta}=\frac{1}{a^{2}(1+e)^{2}} \sqrt{\mu a\left(1-e^{2}\right)}=\sqrt{\frac{\mu}{a^{3}} \frac{(1-e)}{(1+e)^{3}}} .
$$

The mean motion for a circular orbit with radius $a_{\text {circ }}$ is given by

$$
n=\sqrt{\frac{\mu}{a_{\text {circ }}^{3}}}
$$

By setting $\dot{\theta}=n$ we get

$$
a_{\mathrm{circ}}=a \frac{1+e}{(1-e)^{1 / 3}},
$$

where $a_{\text {circ }}$ is the semimajor axis of a circular orbit inferred from the geocentric observation of the apparent motion $\dot{\theta}$ of an object at apogee, which is in an elliptical orbit with semimajor axis $a$ and eccentricity $e$. For a GTO object with $a=24,500 \mathrm{~km}$ and $e=0.7$, i.e., with the apogee at the GEO ring, a radius of $a_{\text {circ }} \approx 62,000 \mathrm{~km}$ would result.

Since July 2002 about half of the survey time has been devoted to the search for objects in GTO orbits with inclinations below $20^{\circ}$. The primary motivation was to find debris of known break-ups of Ariane upper stages. Technically, the only difference between these GTO surveys and our traditional GEO surveys is the telescope tracking during the exposures. While for GEO searches the telescope is tracking with $15^{\prime \prime} / \mathrm{s}$ in right ascension (telescope fixed in the horizon system) we track during GTO surveys either with $7.5^{\prime \prime} / \mathrm{s}$ or $10.5^{\prime \prime} / \mathrm{s}$ - the range of expected apparent motion of GTO objects at apogee. The inferred circular orbits for GTO objects have, obviously, no meaning at all. We therefore try to follow-up objects for which the circular orbit determination yields semimajor axes well outside the range expected for GEO objects. Eventually, a reliable six-parameter orbit could be determined for 332 objects detected in the time period from August 2002 to January 2005.

Figure 38 gives the magnitude distribution for this data set. The magnitudes were corrected for the phase angle but not yet reduced to a common distance. It is therefore not possible to assign an object size to a given magnitude (the indicated sizes are upper limits). The solid line is again indicating the instrument sensitivity. Most of the uncorrelated objects are fainter than magnitude 16 and thus considerably smaller than $1 \mathrm{~m}$ in size. 


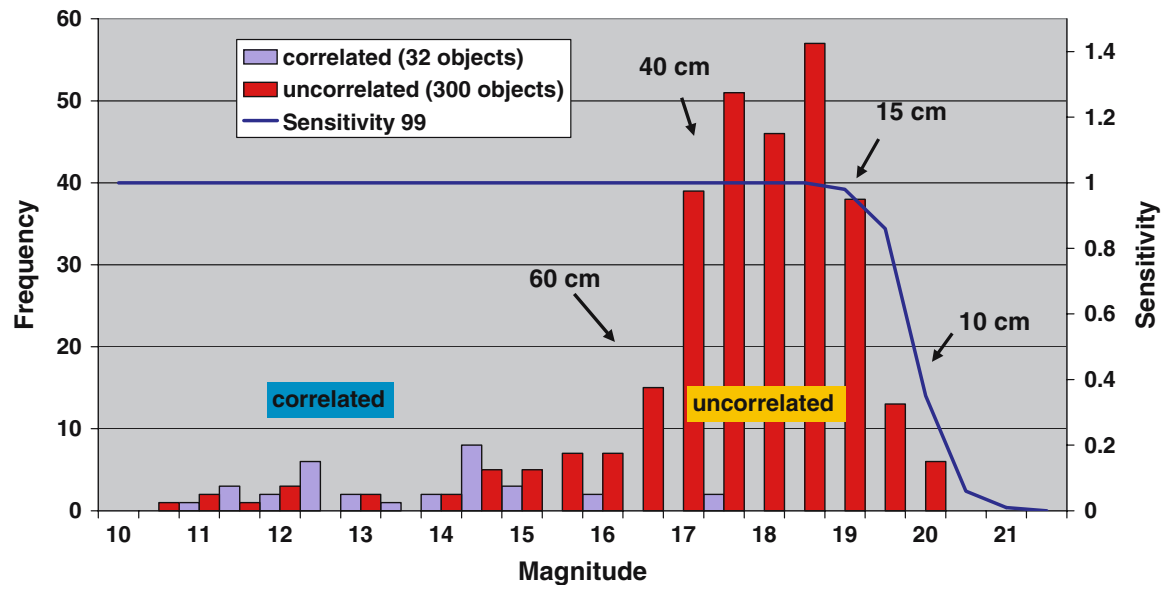

Fig. 38 Magnitude distribution for 332 objects with elliptical orbits

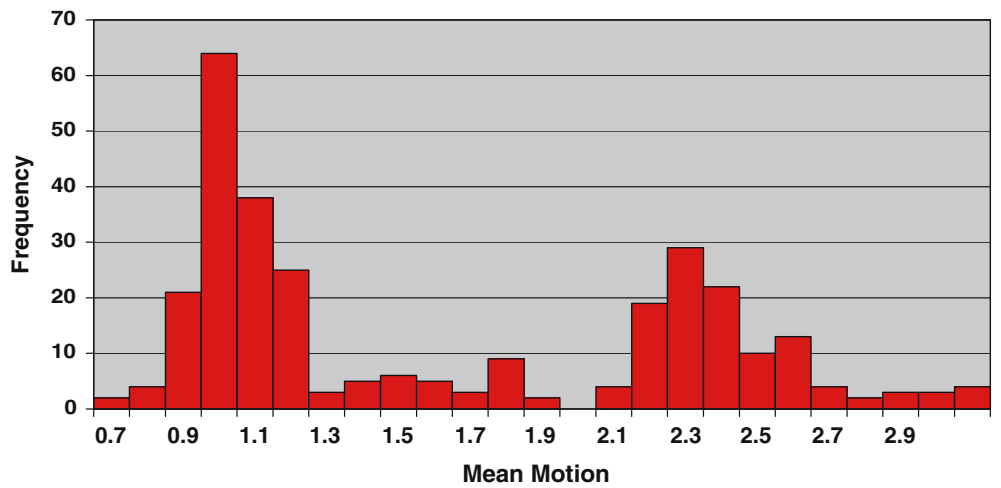

Fig. 39 Distribution of the mean motion for 300 uncorrelated objects with elliptical orbits (Mean motion in rev/day)

The distribution of the mean motion $n$ for the same data set is given in Fig. 39 . There seem to be two maxima: a broad maximum with a peak at mean motion $n=1$ and a second maximum in the range $n=2.1, \ldots, 2.8$. The latter is the typical range for GTO orbits. The corresponding distribution for the objects in the catalog at the epoch 29 April 2004 is given in Fig. 40. (The catalog data was filtered with $e=\{0.1,0.9\}, i=\{0,20.0\}, n=\{0.3,6.0\}$.) The first peak at $n=1$ is completely missing in the catalog data whereas the GTO region is fairly populated.

Figures 41 and 42 show the eccentricity as a function of the mean motion for the same objects and the corresponding catalog data respectively (catalog filtered in the same way as described above).

The lines indicate locations of constant apparent motion in right ascension when the objects are in the apogee. The solid lines (labeled 'vapo $=15^{\prime \prime} / \mathrm{s}$ ', and 'vapo $=5^{\prime \prime} / \mathrm{s}$ ') define the boundaries of the region where the GTO survey was 


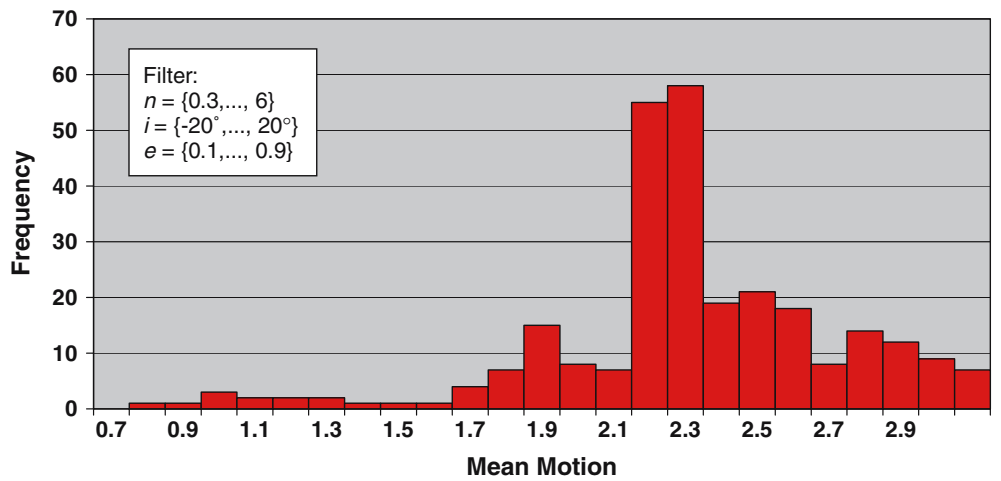

Fig. 40 Distribution of the mean motion of objects in the catalog. (Mean motion in rev/day)

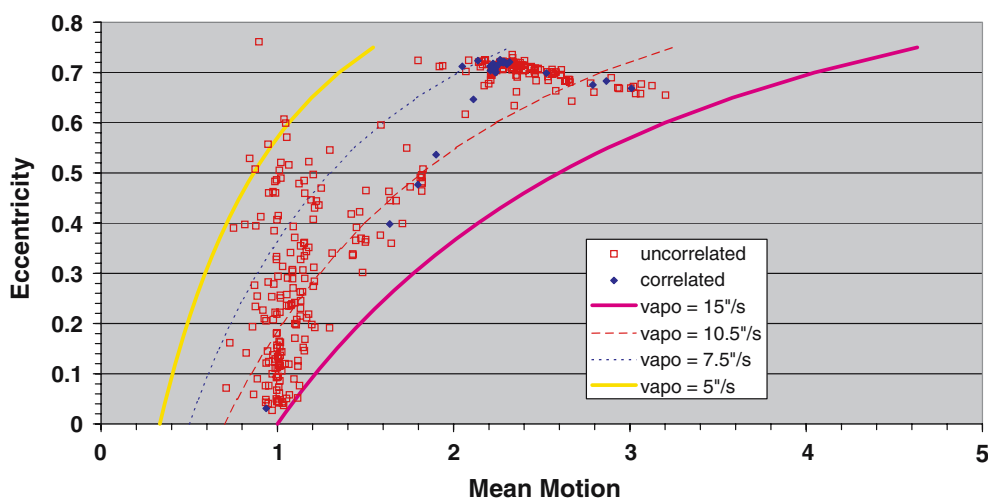

Fig. 41 Eccentricity as a function of the mean motion for 332 objects with elliptical orbits

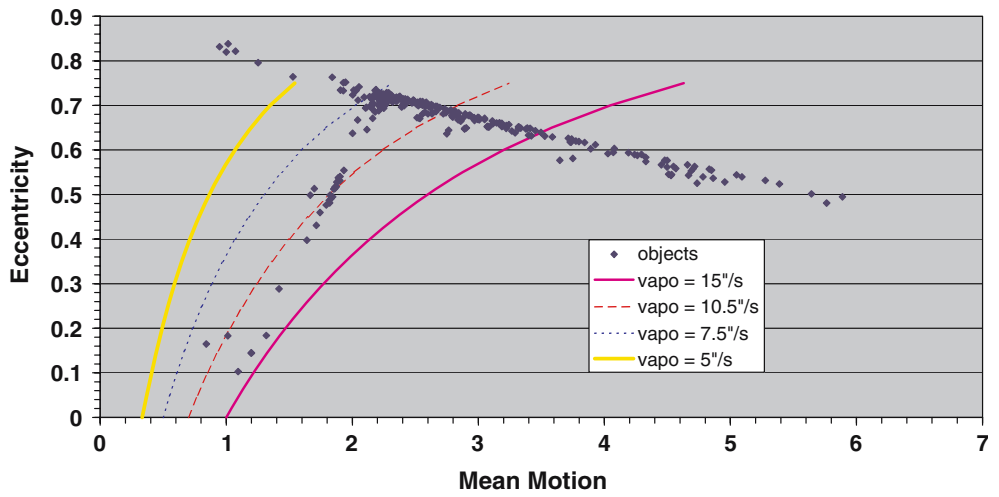

Fig. 42 Eccentricity as a function of the mean motion for the objects in the catalog

able to detect objects. Objects moving slower than about $5^{\prime \prime} / \mathrm{s}$ or faster than $15^{\prime \prime} / \mathrm{s}$ in right ascension would not have passed our detection filter or the subsequent selection criteria to initiate follow-up observations (always assuming that the 
objects are detected near their apogees). The region where the surveys were most sensitive lies between the dotted and the dashed lines. The dotted and the dashed lines correspond to the region of the highest sensitivity of the GTO surveys ('blind tracking' with 7.5 or 10.5 "/ $/ \mathrm{s}$ in right ascension, respectively). The catalog contains mostly objects in GTO, some objects on transfer orbits with the apogee at the nominal GEO altitude but perigee altitudes of $8,000 \mathrm{~km}$ and higher, and very few objects outside these two regions.

In the observed $e-n$ diagram (Fig. 41) we note that (a) there is a population of uncorrelated objects in the region of the GTO orbits (near-horizontal branch at upper right) and (b) that there is a considerable population of uncorrelated objects with a mean motion near one and eccentricities ranging from 0.05 to 0.6 - a region with almost no corresponding objects in the catalog (Fig. 42). This new population of debris objects was completely unexpected and the nature and origin of these objects was unknown at the moment of discovery. As the mean motion, or in other words the orbital energy, of this new population corresponds approximately to the mean motion of objects in GEO, a connection between this population and the GEO population is suspected. Explosive events, however, do not alter the eccentricities of the fragments significantly with respect to the eccentricity of the parent body. Furthermore, there are no or too few potential progenitors in the region of the new population. The only reasonable explanation would be that these objects have a very high area-to-mass ratio and that their originally circular GEO could therefore have been perturbed by radiation pressure resulting in highly eccentric orbits.

We furthermore note that there is a substantial population of debris in the GTO region (Fig. 41).

\subsection{The high area-to-mass ratio population}

There are no potential parent objects in the region of the new population of objects found during the ESA GTO survey. If the objects are explosion debris or debris from disintegrating thermal insulation of spacecrafts they must have been produced in a different orbit region and then been gradually moved into their current orbits by natural perturbations. The mean motion and thus the orbital energy of the objects in the new population is concentrated around the nominal value for GEO objects. An appealing explanation would therefore be that these objects were originally generated in GEO and that their eccentricities have been changed by a (conservative) perturbing force. A corresponding analysis revealed that resonance effects in the gravity field would not produce the observed eccentricities. On the other hand, solar radiation pressure acting on very lightweight objects, i.e., on objects with exceptionally high area-to-mass ratios, could drive GEO objects into orbits with very high eccentricities.

With this in mind we determined area-to-mass ratios for 28 objects of the new population (Fig. 43). Technically, this was done by estimating a direct radiation pressure coefficient in the orbit determination. Standard values of the area-to-mass ratio for entire spacecrafts range from about 0.01 to $0.02 \mathrm{~m}^{2} / \mathrm{kg}$. 


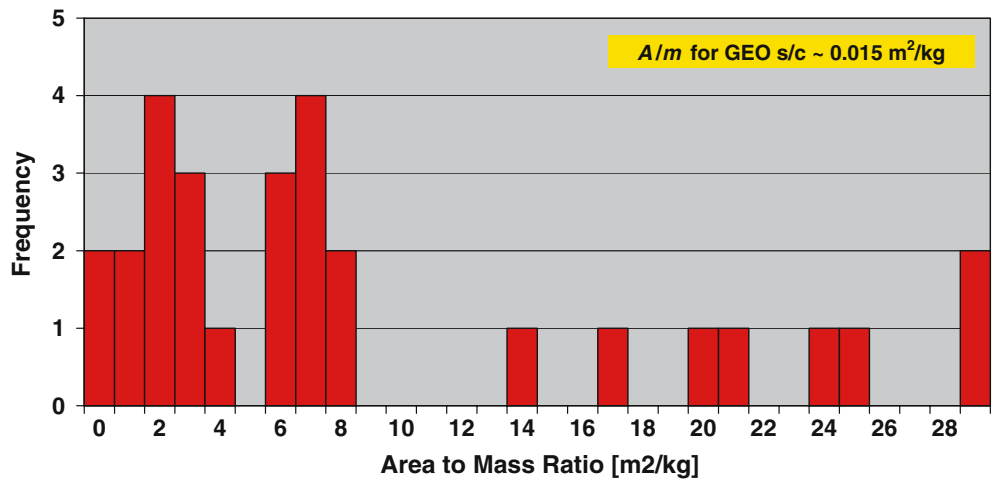

Fig. 43 Distribution of the area-to-mass ratios for 28 uncorrelated objects of the new debris population found during the GTO survey

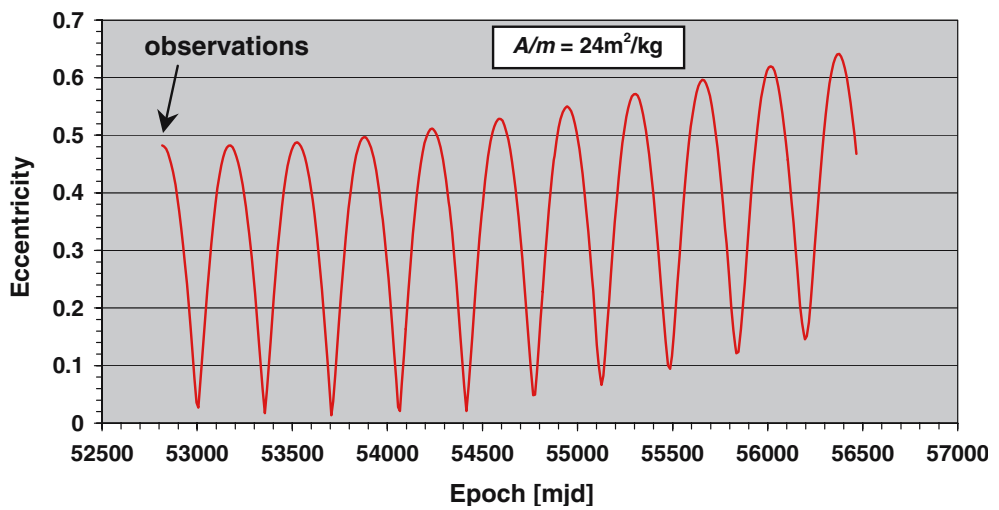

Fig. 44 Evolution of the eccentricity for an object of the new population over the next 10 years

The measured values for the new population are larger by two to three orders of magnitude! These objects must be truly lightweight (a sheet of standard paper has an area-to-mass ratio of about $13 \mathrm{~m}^{2} / \mathrm{kg}$ ). The formal errors of these measurements are less than $10 \%$ of the values in the worst cases. Possible candidate materials are foils used in multilayer insulations of spacecrafts (Liou and Weaver 2005).

The solar radiation pressure is perturbing the orbits of these objects considerably. The main effects are periodic variations of the eccentricity and of the inclination. Figures 44 and 45 show the evolution of the eccentricity and the inclination for one of the objects over the next 10 years. The result was produced by propagating the observed orbit using the estimated area-to-mass ratio of $24 \mathrm{~m}^{2} / \mathrm{kg}$. For more simulations of the long-term evolution of GEO high area-to-mass ratio objects see Anselmo and Pardini (2005).

In order to get an indication on the shape and the surface properties of these objects with high area-to-mass ratios we analyzed the variations in their appar- 


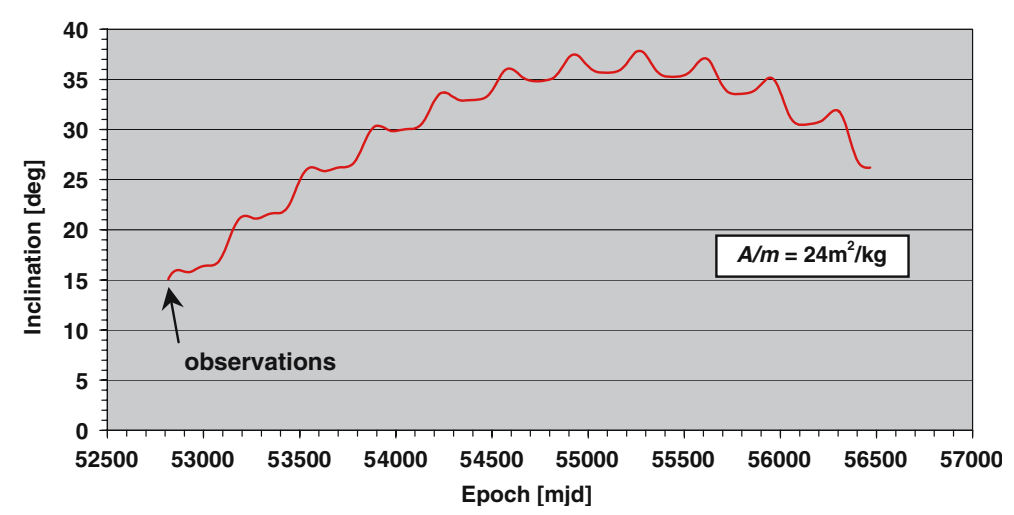

Fig. 45 Evolution of the inclination for an object of the new population over the next 10 years

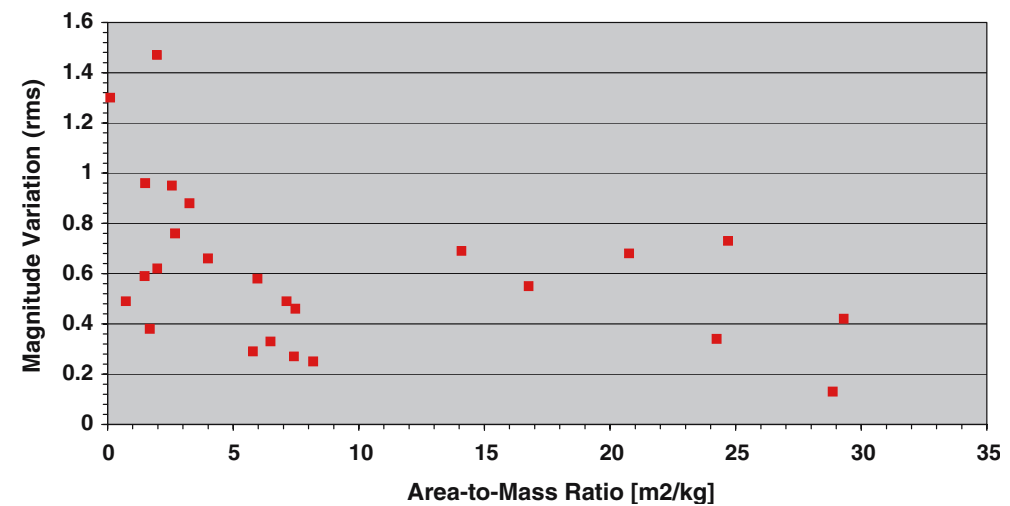

Fig. 46 Variation (rms) of the apparent magnitude as a function of the area-to-mass ratio for 28 uncorrelated objects of the new debris population

ent brightness. Figure 46 shows the variation (rms) of the apparent magnitude as a function of the area-to-mass ratio for 28 uncorrelated objects of the new debris population. There is no obvious correlation between these two parameters. Moreover, the distribution of the brightness variation is very similar to the one observed for normal-type debris with small area-to-mass ratios. The reasonable hypothesis that very lightweight debris would have more plate- or sheet-like shapes than more massive objects is thus not confirmed by this simple analysis.

More information may be obtained from light curves. Some objects with particularly high area-to-mass ratios were therefore observed with the 1-m telescope of the Astronomical Institute of the University of Bern (AIUB) in Zimmerwald, Switzerland. Three resulting light curves for the object 'EGEO021' are given in Fig. 47. All three light curves show significant periodic variations. However, the amplitudes and the periods of these variations are very different in all three cases. The peak-to-peak variations range from 1 

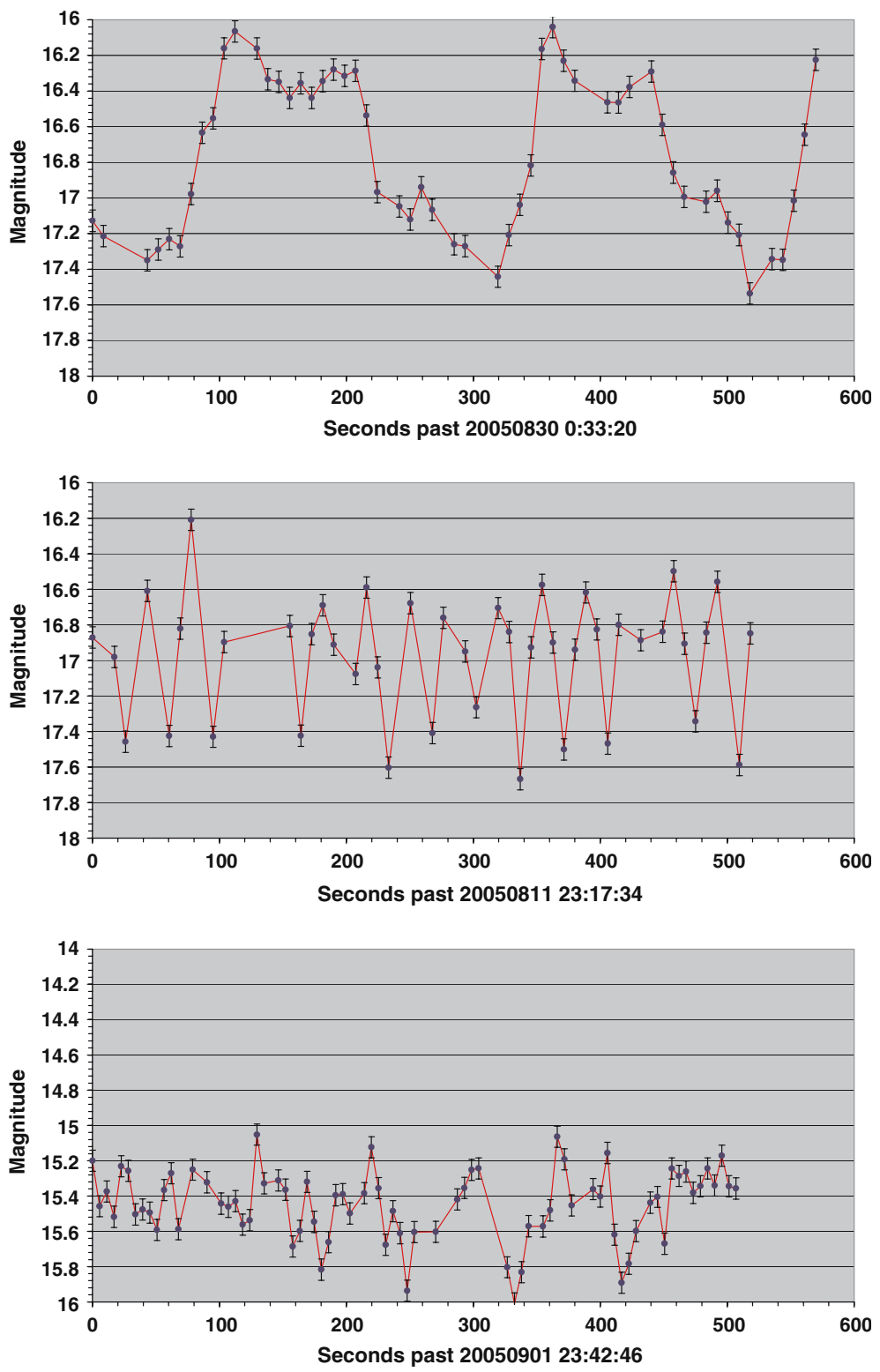

Fig. 47 Three light curves of the object 'EGEO021' which has an area-to-mass ratio of $4.6 \mathrm{~m}^{2} / \mathrm{kg}$

to 1.5 magnitudes and the periods from 35 to $250 \mathrm{~s}$. The apparent magnitude of this object is highly variable-although showing distinct periodic signatures over short time spans of a few minutes-indicating an object in a random tumbling motion with a rather complex shape. An extreme case of another object, which showed no significant variation over a 9-min observation period, 


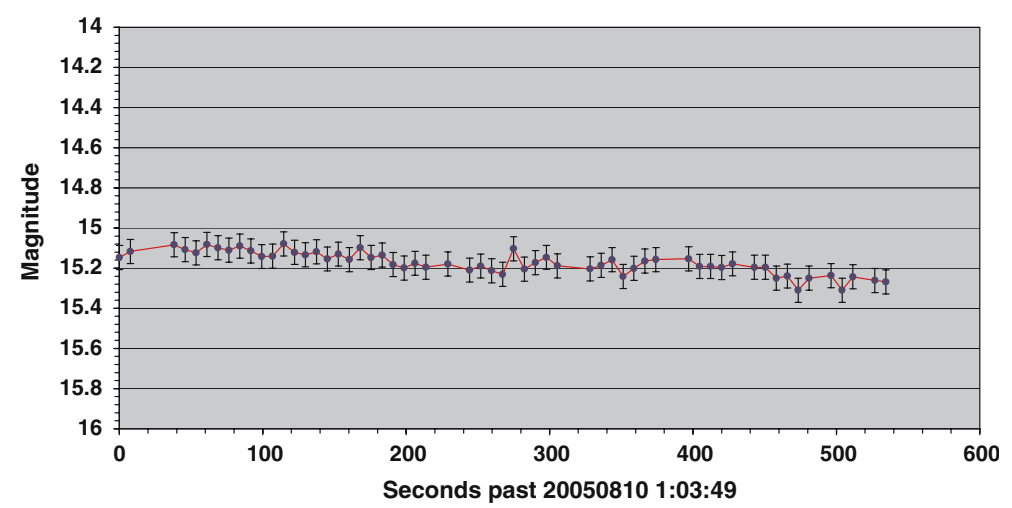

Fig. 48 Light curve of the object 'EGEO014' which has an area-to-mass ratio of $17 \mathrm{~m}^{2} / \mathrm{kg}$

is given in Fig. 48. Obviously, light curves covering much longer time intervals are required to characterize the shapes and the attitude motions of these objects.

\section{Summary}

Space operations inevitably produced debris since the beginning of the space age. The inventory of space debris ranges from large objects like defunct satellites and rocket upper stages over mid-size fragments of explosions or collisions, down to small-sized particles like paint flakes and solid rocket motor slag or dust. This debris poses an increasing threat to operational spacecraft and manned missions. Recognizing the paramount importance of protecting the near-Earth space from contaminating space debris the space agencies initiated programs to measure the current debris population. This data is the prerequisite to develop and validate models, to understand the future evolution of the debris population, to assess the collision risk and to define suitable and cost-efficient mitigation measures.

Optical surveys are best suited to detect space debris in high-altitude orbits in particular in the geostationary ring and in geostationary transfer orbits. The requirements for the optical sensors used in these surveys are strikingly similar to the corresponding demands for systems used in surveys for minor planets, comets and NEOs. The survey techniques developed for space debris searches include specialized hardware, advanced image processing algorithms, techniques to link up observations from moving objects and to determine their orbits, and finally sophisticated data archiving and cataloging techniques. One of the most productive NEO search project, the LINEAR, is based on technologies and techniques that were originally developed for space surveillance, i.e., to detect and track man-made objects in near-Earth space.

The upcoming large synoptic survey system like Pan-STARRS or the LSST will have to base their processing pipelines on enhanced versions of the most 
advanced algorithms developed in the context of space debris and NEO surveys and should fully exploit the experience gained in these fields.

Results from five optical space debris surveys conducted individually by the US space agency NASA, the French space agency CNES and the European space agency ESA have been published so far. The ESA space debris surveythe largest and deepest survey for space debris at high altitudes-revealed a hitherto unknown but substantial population of small debris in GEO. The detection limit of the ESA 1-m telescope at Tenerife, Spain, is of the order of $10 \mathrm{~cm}$ in GEO. The size distribution of the observed population is steadily increasing until the sensitivity limit. Part of this population is also accessible to the other surveys and has been confirmed by them.

There is clear evidence of several debris clouds in the orbital element space. The evolution of about half a dozen clouds could be monitored over the time interval from 2000 to 2005 . The results are consistent with evolution models for debris clouds produced by explosions.

The ESA survey furthermore discovered a completely unexpected, but considerable population of objects with a mean motion near 1 rev/day and eccentricities ranging from 0.05 to 0.6 . Orbit determination provided estimates for the area-to-mass ratio of a small sample of this new population. The measured values are two to three orders of magnitude higher than for standard spacecrafts or 'normal' explosion debris. The new population thus consists of high area-to-mass ratio objects - potentially pieces of multi-layer insulation material-which are driven into orbits with periodically varying eccentricity and inclination by the solar radiation pressure. Photometric light curves of some objects with particularly high area-to-mass ratios were acquired with AIUB's 1-m telescope in Zimmerwald, Switzerland. The apparent magnitudes of most objects are highly variable - although showing distinct periodic signatures over short time spans of a few minutes - indicating objects with complex shapes in a random tumbling motion.

Future optical surveys will have to expand the search regions to other orbital regimes like the medium altitude orbits used by the current and planned global navigation satellite systems. Given the fact that even millimeter-sized objects pose a serious threat to operational satellites is also important to extend the surveys in all regimes to smaller sizes by using larger telescopes or even spacebased sensors. In view of the dynamical nature of the population and in order to detect collisions and explosions a permanent monitoring is required.

Finally, we must face the fact that there is no technically feasible method to clean space from debris in near future. Some unique regions like the GEO region are at the same time most fragile because there is no natural cleaning mechanism like air drag, which would limit the lifetimes of the orbits at these altitudes. The growth of the space debris population is not yet out of control, but actions to substantially mitigate space debris in future are urgently needed. Such mitigation measures will have to be internationally agreed in a binding form and implemented in a consistent manner. This is the only chance we have to preserve near-Earth space in a way that future generations may continue to perform space operations in a technically and economically feasible way. 
Acknowledgements I would like to thank M. C. E. Huber for his invitation to publish this article and for his patience. I am indebted to many colleagues and collaborators, who substantially contributed to the development of the optical observation techniques. I would like to thank in particular Urs Hugentobler, Reto Musci, Martin Ploner, Tim Flohrer and Gerhard Beutler. The ESA program for optical surveys in Tenerife would not exist without the initiatives of and the continuous support by Walter Flury from ESA/ESOC. The program is competitive at an international level, thanks to his leadership. I am grateful to my colleagues for fruitful discussions, in particular to James Dick, Phil Herridge, Pat Seitzer, John Africano and Vladimir Agapov. Stimulating discussions and collaborations with members of the Inter-Agency Space Debris Coordination Committee were also essential. The space debris research at AIUB was supported by several ESA contracts, and by the Swiss National Science Foundation (projects 20-56637.99, 2000-067978.02, and 20020-109527).

\section{References}

Allan RR, Cook GE (1964) The long-period motion of the plane of a distant circular orbit. In: Proc R Soc Lond 280:97-109

Allen CW (1973) Astrophysical quantities. 3rd edn, vol 56. Athlone Press, London, pp 127-243

Anselmo L, Pardini C (2005) Orbital evolution of geosynchronous objects with high area-to-mass ratios. In: 4th European conference on space debris, ESA Publication SP-587, pp 279-284

Africano JL, Schildknecht T (2000) International geostationary observation campaign. Inter-agency space debris coordination committee (IADC) report AI 12.1

Africano JL, Schildknecht T (eds) (2003) International space debris measurement campaigns in GEO. Inter-agency space debris coordination committee (IADC) Report AI 18.2

Africano JL, Schildknecht T (eds) (2004) Follow-on international space debris measurement campaigns in GEO. Inter-agency space debris coordination committee (IADC) report AI 20.2

Africano JL et al. (1999) Liquid mirror telescope observations of the orbital debris environment. NASA JSC report JSC-28826

Africano JL, Sydney PF, Hamada KM, Soo Hoo V (2001) Deep-space satellite observations using the near-Earth asteroid tracking (NEAT) camera at AMOS. Proc SPIE 4490:187-193

Africano JL, Stansbery EG, Kervin PW (2004) The optical orbital debris measurement program at NASA and AMOS. In: 34th COSPAR scientific assembly, October 10-19, 2002, Houston, Texas, USA. Adv Sp Res 34(5):892-900

Ariane 4 User's Manual Issue No.2, Arianespace, February 1992

Ariane 5 User's Manual Issue No.3 Revision 0, Arianespace, March 2000

Barker E, Jarvis K, Africano J, Jorgensen K, Parr-Thumm T, Matney M, Stansbery G (2005) The GEO environment as determined by the CDT between 1998 and 2002. In: 4th European conference on space debris, ESA Publication SP-587, pp 135-140

Bendisch J, Bunte KD, Krag H, Sdunnus H, Wegener P, Walker R, Wiedemann C (2002) Upgrade of ESA's MASTER Model. Final Report, ESA ESOC contract 14710/00/D/HK

Bendisch J, Bunte K, Klinkrad H, Krag H, Martin C, Sdunnus H, Walker R, Wegener P, Wiedemann C (2004) The MASTER-2001 model. Adv Sp Res 34(5):959-968

Bertin E. Sextractor User manual, http://terapix.iap.fr/IMG/pdf/sextractor.pdf

Beutler G (2004) Methods of celestial mechanics: basic principles and applications. Springer, Berlin Heidelberg New York

Bohannon GE, Young N (1993) Debris size estimation using average RCS measurements. Report no. 930781-BE-2247, XonTech, Inc., Los Angeles, CA, September 1993

Flury W (1988) The situation in the geostationary orbit. Workshop on environmental activities in outer space: state of the law and protection. Cologne, Germany, May 16-19

Flury W (1991) Collision probability and spacecraft deposition in the geostationary orbit. Adv Sp Res 11(12):67-79

Flury W, Hugentobler U, Kuusela J, Schildknecht T, Sodnik Z (2000) The search and discovery of small-size debris in the geostationary ring with the Zeiss 1 meter telescope. ESA Bulletin 104

Henize K, Stanley J (1990) Optical observations of space debris. AIAA-90-1340

Hernández C, Jehn R (2003) Classification of geosynchronous Objects Issue 5, ESOC, January 2003

Høg E, Fabricius C, Makarov VV, Urban S, Corbin T, Wycoff G, Bastian U, Schwekendiek P, Wicenec A (1995) The Tycho-2 catalog of the 2.5 million brightest stars. Astron Astrophys 355:L27-L30 
IAA Position Paper on Orbital Debris (1993) Acta Astronautica, vol 31, pp 169-191, Pergamon Press

IAA Position Paper on Orbital Debris (2001) updated version of IAA position paper on orbital debris 1993

Interagency Report on Orbital Debris (1995) Office of science and technology policy, The National Science and Technology Council

Isobe S, Mulherin J, Way S, Downey E, Nishimura K, Doi I, Saotome M (2000) A cost-effective, advanced-technology telescope system for detecting near-earth objects and space debris. In: Proceedings of SPIE on Telescope Structures, Enclosures, Controls, Assembly/Integration/Validation and Commissioning, vol. 4004, 2000

Isobe S, Asami A, Asher D, Hashimoto N, Nakano S-I, Nishiyama K, Ohshima Y, Terazono J, Umehara H, Urata T, Yoshikawa M (2004) A new $1 \mathrm{~m}$ telescope for space debris survey observations. Adv Sp Res 34(5):917-920

Jarvis KS et al. (2002) CCD debris telescope observations of the geosynchronous orbital debris environment, observing Year 1999. NASA JSC report JSC-29537

Kessler DJ, Jarvis KS (2004) Obtaining the properly weighted average albedo of orbital debris from optical and radar observations. In: Proceedings of the 34th COSPAR scientific assembly, October 11-18, 2002, Houston TX, USA. Adv Sp Res 34(5):1006-1012

Kessler DJ, Matney MJ et al (1997) The search for a previously unknown source of orbital debris: the possibility of a coolant leak in radar reconnaissance satellites. NASA JSC report JSC-27737

Larson S, Brownlee J, Hergenrother C, Spahr T (1998) The Catalina sky survey for NEOs. Bull Am Astron Soc 30:1037ff

Larsen J, Gleason AE, Danzl NM, Descour AS, McMillan RS, Gehrels T, Jedicke R, Montani JL, Scotti JV (2001) The spacewatch wide-area survey for bright centaurs and trans-neptunian objects. Astron J 121:562-579

Liou J-C, Weaver JK (2005) Orbital dynamics of high area-to-mass ratio debris and their distribution in the geosynchronous region. In: 4th European conference on space debris, ESA Publication SP-587, pp 285-290

Lobb DR, Dick JSB, Green SF (1993) Development of concepts for detection and characterisation of debris in Earth orbit using passive optical instruments. Adv Sp Res 13(8):59-63

Mehrholz D, Leushacke L, Banka D (2004) Beam-park experiments at FGAN. In: Proceedings of the 34th COSPAR scientific assembly, october 11-18, 2002, Houston TX, USA, Adv Sp Res 34(5):863-871

Musci R, Schildknecht T, Flohrer T, Beutler G (2005) Concept for a catalog of space debris in GEO. In: 4th European conference on space debris, April 18-20, ESOC, Darmstadt, Germany, ESA Publication SP-587, pp 601-606

Pardini C, Anselmo L (2005) Dynamical evolution of debris clouds in geosynchronous orbit. In: 35th COSPAR scientific assembly, 18-25, July 2004, Paris, France. Adv Sp Res 35(7):1302-1312

Perryman MAC, Lindegren L, Kovalevsky J, Høg E, Bastian U, Bernacca PL, Crézé M, Donati F, Grenon M, Grewing M, van Leeuwen F, van der Marel H, Mignard F, Murray CA, Le Poole RS, Schrijver H, Turon C, Arenou F, Froeschlé M, Petersen CS (1997) The HIPPARCOS catalog Astron Astrophys 323:L49-L52

Pravdo SH, Rabinowitz DL, Helin EF, Lawrence KJ, Bambery RJ, Clark, Groom SL, Levin S, Lorre J, Shaklan SB, Kervin P, Africano JA, Sydney P, Soohoo V (1999) The near-earth asteroid tracking (NEAT) program: An automated system for telescope control, wide-field imaging, and object detection. Astron J 117:1616-1633

Ríos Bergantiños S, Deguine B, Klotz A, Thiebau C, Foliard J, Boër M (2005) Improvement of the TAROT system used for space debris optical observations and observation campaign results. In: 4th European conference on space debris, ESA publication SP-587, pp 125-130

Schildknecht T, Hugentobler U, Verdun A (1995a) Algorithms for ground based optical detection of space debris. In: Proceedings of 30th COSPAR scientific assembly, Hamburg, Germany 11-21 July 1994. Adv Sp Res 16(11):47-50

Schildknecht T, Beutler G, Hugentobler U, Verdun A (1995b) CCD algorithms for space debris detection. Final Report, ESA ESOC Contract 10623/93/D/IM

Schildknecht T, Hugentobler U, Fridez P, Kunszt P (1998) CCD off-line data processing system. Executive Summary, ESA ESOC Contract 11435/95/D/IM 
Schildknecht T, Musci R, Ploner M, Kuusela J, de Leon Cruz J, de Fatima Dominguez Palmero L (2001a) Optical surveys for space debris in GEO - what about NEOs? In: Isobe S (ed) International workshop on collaboration and coordination among NEO observers and orbital computers, 23-26, October 2001, Kurashiki, Japan, pp 121-132

Schildknecht T, Ploner M, Fuentes O (2001b) Level-1 telescope control and on-line data processing system. Final report phase A, ESA ESOC Contract 12166/96/D/IM

Schildknecht T, Musci R, Ploner M, Beutler G, Flury W, Kuusela J, de Leon Cruz J, de Fatima Dominguez Palmero L (2004) Optical observations of space debris in GEO and in highly-eccentric orbits. In: 34th COSPAR scientific assembly, October 10-19, 2002, Houston, Texas, USA. Adv Sp Res 94(5):901-911

Seitzer P, Jorgensen K, Africano JL, Parr-Thumm T, Matney M, Jarvis K, Stansbery E, Barker E (2005) An optical survey for GEO debris in high inclination orbits. In: Proceedings of the AMOS technical conference, September 5-9, Maui, Hawaii, pp 224-229

Settecerri TJ, Stansbery EG, Herbert TJ (1999) Radar measurements of the orbital debris environment: Haystack and HAX Radars October 1990 - October 1998. NASA JSC report JSC-28744

Space Debris (1988) Report of the ESA Space Debris Working Group, ESA SP-1109, Paris

Stokes GH, Evans JB (2002) Near-earth asteroid search programs. Asteroids III, Bottke WF et al. (eds) pp. $45-54$

Stokes GH, Evans JB, Viggh EM, Shelly FC, Pearce EC (2000) Lincoln near-earth asteroid program (LINEAR). Icarus 148:21-28

Talent D, Potter A, Henize K (1997) Report on the NASA/Schmidt GEO survey program. In: Proceedings of the 2nd European conference on space debris, Flury W (ed), Darmstadt, Germany, March17-19, 1997, ESA SP-393, pp 99-104

UN Technical Report on Space Debris, (1999) Scientific and Technical Subcommittee of the United Nations Committee on the Peaceful Uses of Outer Space, New York

Wegener P, Bendisch J, Wiedemann C (2001) The Reference population of the MASTER 2001 Model. In: 52nd IAF Congress, Toulouse, France, October 1-5 